\title{
Dois resultados em combinatória contemporânea
}

\author{
Guilherme Oliveira Mota
}

TESE APRESENTADA

$\mathrm{AO}$

Instituto DE Matemática e Estatística

DA

Universidade de SÃo Paulo

PARA

OBTENÇÃO DO TÍTULO

$\mathrm{DE}$

Doutor EM CiÊnCIAS

\author{
Programa: Ciência da Computação \\ Orientador: Prof. Dr. Yoshiharu Kohayakawa
}

Durante o desenvolvimento deste trabalho o autor recebeu auxílio financeiro do CNPq (140882/2009-0) e da FAPESP (2009/06294-0 e 2012/00036-2) 


\section{Dois resultados em combinatória contemporânea}

Esta é a versão original da tese elaborada pelo candidato Guilherme Oliveira Mota, tal como submetida à Comissão Julgadora. 


\section{Agradecimentos}

Primeiramente agradeço a meus pais, que nunca pouparam esforços para que eu tivesse uma formação de qualidade, sempre me apoiando e me ajudando em tudo e, apesar de estarem tão longe, sempre fazem o possível para ficarem mais próximos de mim. Agradeço também ao meu irmão George e ao meu primo Fábio (um irmão para mim) pelos vários momentos de alegria que me proporcionam sempre que vou a Fortaleza.

Não posso deixar de prestar meus sinceros agradecimentos ao Yoshi, que sempre me ofereceu oportunidades para que eu pudesse crescer intelectualmente. Além disso, suas qualidades de excelente professor e pesquisador servem constantemente como fonte de inspiração para mim e me motivam a continuar buscando melhorar.

Entre muitas coisas boas que me aconteceram em São Paulo durante o meu doutorado, certamente uma das melhores foi conhecer a Suelen, essa pessoa maravilhosa na qual tenho o prazer de ter como minha companheira. Sussu teve um papel fundamental durante o desenvolvimento da minha pesquisa, sempre me incentivando e me apoiando nos momentos mais difíceis, sendo o alicerce que me manteve firme ao longo desses últimos anos. Ressalto também sua contribuição importantíssima na revisão final da tese.

Muitos amigos também me ajudaram durante a realização dessa tese. Sou muito grato ao Leandro Lima, por ter me incentivado a fazer doutorado na USP. Um agradecimento especial vai para Roberto e Rafinha, que estiveram sempre presentes, ajudando nos momentos difíceis e tornando os momentos felizes ainda mais especiais. Agradeço à Naty, essa pessoa querida que veio se juntar ao trio cearense. Não posso deixar de agradecer também à Iasmin, Patrícia e Tixa, pelo carinho e pelas longas conversas, especialmente quando eu me encontrava longe do país.

Agradeço de todo o coração à nova família que ganhei na Alemanha, em Hamburgo: André e Philip, por serem parceiros para toda hora, irmãos brasileiros que ganhei na Alemanha, e Tia Dô, por ter me acolhido como um filho em sua casa, com a alegria e carinho que lhe é peculiar.

Sou também muito agradecido aos meus orientadores alemães, Anusch e Mathias, por terem me ajudado no desenvolvimento de meu projeto de pesquisa e por terem me recebido tão bem na Alemanha.

Por fim, agradeço a todos os parceiros de jiu-jitsu que tive durante esses anos, especialmente ao meu mestre Adriano Silva. Certamente os treinos foram essenciais para que eu mantivesse a mente leve para trabalhar melhor nos problemas matemáticos. 


\section{Resumo}

Mota, G. O. Dois resultados em combinatória contemporânea. Tese - Instituto de Matemática e Estatística, Universidade de São Paulo, São Paulo, 2013.

Dois problemas combinatórios são estudados: (i) determinar a quantidade de cópias de um hipergrafo fixo em um hipergrafo uniforme pseudoaleatório, e (ii) estimar números de Ramsey de ordem dois e três para grafos com largura de banda pequena e grau máximo limitado.

Apresentamos um lema de contagem para estimar a quantidade de cópias de um hipergrafo $k$-uniforme linear livre de conectores (conector é uma generalização de triângulo, para hipergrafos) que estão presentes em um hipergrafo esparso pseudoaleatório $G$. Considere um hipergrafo $k$-uniforme linear $H$ que é livre de conectores e um hipergrafo $k$-uniforme $G$ com $n$ vértices. Seja $d_{H}=\max \{\delta(J): J \subset H\}$ e $D_{H}=\min \left\{k d_{H}, \Delta(H)\right\}$. Estabelecemos que, se os vértices de $G$ não possuem grau grande, famílias pequenas de conjuntos de $k-1$ elementos de $V(G)$ não possuem vizinhança comum grande, e a maioria dos pares de conjuntos em $\left(\begin{array}{c}V(G) \\ k-1\end{array}\right)$ possuem a quantidade "correta" de vizinhos, então a quantidade de imersões de $H$ em $G$ é $(1+o(1)) n^{|V(H)|} p^{|E(H)|}$, desde que $p \gg n^{1 / D_{H}}$ e $|E(G)|=\left(\begin{array}{l}n \\ k\end{array}\right) p$. Isso generaliza um resultado de Kohayakawa, Rödl e Sissokho [Embedding graphs with bounded degree in sparse pseudorandom graphs, Israel J. Math. 139 (2004), 93-137], que provaram que, para $p$ dado como acima, esse lema de imersão vale para grafos, onde $H$ é um grafo livre de triângulos.

Determinamos assintoticamente os números de Ramsey de ordem dois e três para grafos bipartidos com largura de banda pequena e grau máximo limitado. Mais especificamente, determinamos assintoticamente o número de Ramsey de ordem dois para grafos bipartidos com largura de banda pequena e grau máximo limitado, e o número de Ramsey de ordem três para tais grafos, com a suposição adicional de que ambas as classes do grafo bipartido têm aproximadamente o mesmo tamanho.

Palavras-chave: Pseudoaleatoriedade, hipergrafos, imersão, Ramsey, regularidade. 


\section{Abstract}

Mota, G. O. Two results in modern combinatorics. Tese - Instituto de Matemática e Estatística, Universidade de São Paulo, São Paulo, 2013.

Two combinatorial problems are studied: (i) determining the number of copies of a fixed hipergraph in uniform pseudorandom hypergraphs, and (ii) estimating the two and three color Ramsey numbers for graphs with small bandwidth and bounded maximum degree.

We give a counting lemma for the number of copies of linear $k$-uniform connector-free hypergraphs (connector is a generalization of triangle for hypergraphs) that are contained in some sparse hypergraphs $G$. Let $H$ be a linear $k$-uniform connector-free hypergraph and let $G$ be a $k$-uniform hypergraph with $n$ vertices. Set $d_{H}=\max \{\delta(J): J \subset H\}$ and $D_{H}=\min \left\{k d_{H}, \Delta(H)\right\}$. We proved that if the vertices of $G$ do not have large degree, small families of $(k-1)$-element sets of $V(G)$ do not have large common neighbourhood and most of the pairs of sets in $\left(\begin{array}{c}V(G) \\ k-1\end{array}\right)$ have the 'right' number of common neighbours, then the number of embeddings of $H$ in $G$ is $(1+o(1)) n^{|V(H)|} p^{|E(H)|}$, given that $p \gg n^{1 / D_{H}}$ and $|E(G)|=\left(\begin{array}{l}n \\ k\end{array}\right) p$. This generalizes a result by Kohayakawa, Rödl and Sissokho [Embedding graphs with bounded degree in sparse pseudorandom graphs, Israel J. Math. 139 (2004), 93137], who proved that, for $p$ as above, this result holds for graphs, where $H$ is a triangle-free graph.

We determine asymptotically the two and three Ramsey numbers for bipartite graphs with small bandwidth and bounded maximum degree. More generally, we determine asymptotically the two color Ramsey number for bipartite graphs with small bandwidth and bounded maximum degree and the three color Ramsey number for such graphs with the additional assumption that both classes of the bipartite graph have almost the same size.

Keywords: Pseudorandomness, hypergraphs, embedding, Ramsey, regularity. 


\section{Sumário}

$\begin{array}{lll}1 & \text { Introdução } & 1\end{array}$

2 Preliminares $\quad 5$

2.1 Grafos . . . . . . . . . . . . . . . . . . . . . . . . 5

2.2 Hipergrafos $\ldots \ldots \ldots \ldots \ldots \ldots$

\begin{tabular}{|lll}
\hline & Lema de contagem para hipergrafos pseudoaleatórios & 9
\end{tabular}

3.1 Lemas principais . . . . . . . . . . . . . . . . . . . . 11

3.2 Resultados auxiliares . . . . . . . . . . . . . . . . . . . . . . . . . . 12

3.2 .1 Alguns resultados combinatórios . . . . . . . . . . . . . . . . 12

3.2 .2 Generalizando as propriedades LMT e CG . . . . . . . . . . . . . . . 13

3.3 Lema de Extensão e corolários . . . . . . . . . . . . . . . . . . . . . . . . . . . 19

3.3 .1 Lema de Extensão $\ldots \ldots \ldots$. . . . . . . . . . . . . . . . . . . 19

3.3 .2 Corolários do Lema de Extensão . . . . . . . . . . . . . . . . . . . 21

3.4 Provas dos lemas principais . . . . . . . . . . . . . . . . . . . . . 24

3.4 .1 Prova do Lema $\mathrm{CG}_{2 \rightarrow t} \ldots \ldots \ldots \ldots \ldots$. . . . . . . . . . . 24

3.4 .2 Prova do Lema de contagem modificado . . . . . . . . . . . . . 27

4 Números de Ramsey para grafos bipartidos 31

4.1 Visão geral da prova do Teorema 3-Ramsey . . . . . . . . . . . . . . . . . . . 33

4.2 Resultados auxiliares . . . . . . . . . . . . . . . . . . . . . . . . . . 35

4.2 .1 Método da Regularidade . . . . . . . . . . . . . . . . . . 36

$4.2 .2 \quad$ Explosão regular de uma árvore $\ldots \ldots$. . . . . . . . . . . . . . . . . . . . . . 38

4.2 .3 Intervalos balanceados . . . . . . . . . . . . . . . . . . . 40

4.3 Prova do Teorema 3-Ramsey . . . . . . . . . . . . . . . . . . . . . . . . 43

4.4 Ideia da prova do Teorema 2-Ramsey . . . . . . . . . . . . . . . . . . . . 48

$\begin{array}{lll}5 & \text { Considerações finais } & 51\end{array}$

\begin{tabular}{ll}
\hline Referências Bibliográficas & 53
\end{tabular} 


\section{Capítulo 1}

\section{Introdução}

Uma classe de problemas particularmente importante em combinatória diz respeito a determinar se existe uma cópia de um grafo fixo em grafos maiores. O clássico Lema de Regularidade de Szemerédi [53] afirma que, desde que um grafo seja suficientemente grande, seu conjunto de vértices pode ser particionado em no máximo um número constante de classes com aproximadamente o mesmo tamanho, de modo que as arestas, na maioria dos grafos bipartidos induzidos pelos vértices dessas classes e pelas arestas entre as classes, são bem distribuídas (uma partição satisfazendo essa propriedade é dita regular). Porém, dado um grafo $G=(V, E)$ com $n$ vértices, a partição de $V$ obtida pelo Lema de Regularidade contém uma quantidade quadrática de pares não regulares, de modo que, se a quantidade de arestas do grafo em questão é $o\left(n^{2}\right)$, elas podem estar quase totalmente contidas somente nos pares não regulares. Nesse caso, o subgrafo de $G$ induzido pelos pares regulares é muito esparso. Logo, o Lema de Regularidade é útil somente para grafos densos (grafos com $\Omega\left(n^{2}\right)$ arestas). Para uma descrição mais formal do Lema de Regularidade, veja a Seção 4.2.1.

Lemas de Imersão são resultados que garantem a existência de cópias de grafos fixos $H$ em grafos maiores, tipicamente em partições regulares. Quando, em vez de garantir somente a existência, a quantidade de cópias de $H$ é estimada, temos o que chamamos de Lemas de Contagem. Assim, a combinação do Lema de Regularidade com lemas de contagem é muito útil na solução de problemas que objetivam encontrar cópias de um grafo $H$ em grafos maiores. Para uma discussão aprofundada sobre regularidade, lemas de contagem para grafos densos e aplicações, veja [40, 43].

O grafo aleatório binomial de Erdös e Rényi $G \in G(n, p)$ é um grafo com um conjunto de $n$ vértices, onde cada dois vértices são adjacentes com probabilidade $p$ e essas adjacências são independentes. Dado um grafo $H$, a 2-densidade de $H$ é dada por $d_{2}(H)=$ $(|E(H)|-1) /(|V(H)|-2)$. Um grafo $H$ é dito 2-balanceado se a 2-densidade de $H$ é maior ou igual que a 2-densidade de qualquer de seus subgrafos que contêm pelo menos três vértices. Kohayakawa e Rödl introduziram uma versão do Lema de Regularidade para grafos esparsos, i.e., grafos com uma quantidade subquadrática de arestas (veja, e.g. [33, 36]). Todavia, durante muito tempo, não se conheciam lemas de contagem que funcionassem nesse 
contexto esparso. Kohayakawa, Łuczak e Rödl [35] conjecturaram um resultado de contagem probabilístico, conhecido como Conjectura $K \notin R$, que, durante muito tempo foi sido provado apenas para algumas classes particulares de grafos (veja [23, 25, 26, 34, 38]). Em 2012, foi provada uma versão da Conjectura KŁR para subgrafos de $G(n, p)$ [15], uma versão para grafos 2-balanceados [2] e, finalmente, a conjectura foi provada em toda sua generalidade no trabalho de Saxton e Thomason [52], através de uma técnica de contagem de conjuntos independentes em hipergrafos uniformes.

Pelo que foi discutido nos parágrafos anteriores, já sabemos da utilidade de lemas de contagem e sabemos também que os problemas relacionados a esses lemas têm sido extensivamente estudados desde o surgimento do Lema de Regularidade. Em particular, um problema que tem chamado a atenção dos pesquisadores é a obtenção de lemas de contagem de grafos fixos em grafos "pseudoaleatórios", como pode ser visto nos trabalhos de Conlon, Fox e Zhao [13], e Kohayakawa, Rödl, Schacht e Skokan [39]. Diante desses resultados envolvendo grafos, o interesse na resolução de problemas para hipergrafos cresceu de forma significativa (veja [14, 21, 22]). Um dos problemas em que estamos interessados aqui é a investigação de lemas de contagem de hipergrafos fixos em hipergrafos pseudoaleatórios.

Existem, na literatura, diversas noções bem conhecidas de pseudoaleatoriedade para grafos (veja, e.g., [10, 9, 44, 54]). Algumas dessas noções são relacionadas com o grau e cograu dos vértices, e com a distribuição das arestas do grafo. Tais conceitos de pseudoaleatoriedade foram generalizados de algumas maneiras diferentes para hipergrafos (veja [21, 22]). Em [21], intuitivamente, um hipergrafo uniforme $G$ é definido como pseudoaleatório se, (i) para todo conjunto 'pequeno' de vértices $S$, a quantidade de arestas que contêm $S$ é próxima da quantidade esperada em um hipergrafo uniforme aleatório, e (ii) para todo par de conjuntos 'pequenos' de vértices $S_{1}$ e $S_{2}$, o cograu de $S_{1}$ e $S_{2}$, i.e., a quantidade de arestas de $G$ que contêm $S_{1}$ e $S_{2}$, não é muito maior do que a quantidade esperada em um hipergrafo uniforme aleatório. Em [22], a noção de pseudoaleatoriedade considerada é mais forte, no sentido que é preciso ter o controle não somente dos graus e cograus dos vértices, mas de algumas outras estruturas do hipergrafo. No Capítulo 3 discutimos uma noção de pseudoaleatoriedade para hipergrafos, que se assemelha à noção utilizada em [21], e provamos um lema de contagem em hipergrafos suficientemente pseudoaleatórios.

Dado um grafo $H$, defina $d_{H}=\max \{\delta(J): J \subset H\}$ e $D_{H}=\min \left\{2 d_{H}, \Delta(H)\right\}$, onde $\delta(H)$ e $\Delta(H)$ são, respectivamente, os graus mínimo e máximo de $H$. Em [37], Kohayakawa, Rödl e Sissokho provaram que, dado um grafo fixo $H$ livre de triângulos e $p=o(1)$ com $p \gg n^{-1 / D_{H}}$ vale o seguinte. Para todo $\varepsilon>0$, existem $\delta>0$ tal que se $G$ é um grafo com $n$ vértices suficientemente grande, onde $\left|E_{G}\right|=\left(\begin{array}{l}n \\ 2\end{array}\right) p$, a vizinhança comum de subconjuntos pequenos de vértices de $G$ não é 'grande' e, para a maioria dos vértices $v$ e pares de vértices $\{u, v\}$ de $G$, o grau de $v$ e o cograu de $\{u, v\}$ é próximo do valor esperado em $G(n, p)$, então o seguinte vale: || $\mathcal{I}(H, G)\left|-n^{v(H)} p^{e(H)}\right|<\varepsilon n^{v(H)} p^{e(H)}$, onde $\mathcal{I}(H, G)$ é o conjunto de todas as imersões de $H$ em $G$. O lema de contagem apresentado no Capítulo 3 generaliza o resultado de Kohayakawa, Rödl e Sissokho para hipergrafos lineares $k$-uniformes, onde estimamos a 
quantidade de imersões de um hipergrafo linear (duas arestas compartilham no máximo um vértice) $k$-uniforme livre de conectores em hipergrafos $k$-uniformes pseudoaleatórios, onde um conector é uma aresta $e \in E_{H}$ tal que existem $x \in V_{H} \backslash\{e\}$ e $k$ arestas $e_{1}, \ldots, e_{k}$ que contêm $x$, com $\left|e \cap e_{i}\right|=1$ para todo $i=1, \ldots, k$.

Outro problema investigado nesta tese diz respeito à Teoria de Ramsey. Em 1930, Ramsey provou um importante e clássico resultado, que é conhecido pelo nome de "Teorema de Ramsey" [48], dando origem a uma área de pesquisa que hoje é conhecida por Teoria de Ramsey. O teorema provado por Ramsey, que é uma generalização do princípio da casa dos pombos, diz que, para todo par de inteiros $\{r, s\}$, existe um inteiro $n$ tal que todo grafo completo com pelo menos $n$ vértices, cujas arestas são coloridas com duas cores (digamos, azul e vermelho), possui uma cópia do grafo completo com $r$ vértices, onde todas as arestas são azuis, ou possui uma cópia do grafo completo com $s$ vértices, onde todas as arestas são vermelhas. $\mathrm{O}$ menor desses inteiros $n$ tal que vale a propriedade acima é chamado de número de Ramsey e denotado por $R(r, s)$. Uma generalização natural dos números de Ramsey surge quando, em vez de grafos completos com $r$ e $s$ vértices, consideramos grafos arbitrários $G_{1}$ e $G_{2}$. Denotamos por $R\left(G_{1}, G_{2}\right)$ o menor inteiro tal que todo grafo completo com pelo menos $R\left(G_{1}, G_{2}\right)$ vértices, cujas arestas são coloridas com as cores azul e vermelho, possui uma cópia azul de $G_{1}$ ou uma cópia vermelha de $G_{2}$. Dizemos que $R\left(G_{1}, G_{2}\right)$ é o número de Ramsey de ordem 2 para $G_{1}$ e $G_{2}$.

Podemos generalizar também a noção de números de Ramsey permitindo mais cores na coloração. Definimos o número de Ramsey de ordem $r$, denotado por $R\left(G_{1}, G_{2}, \ldots, G_{r}\right)$, como o menor inteiro positivo $n$ tal que, se as arestas de um grafo completo com $n$ vértices são particionadas em $r$ classes de cores distintas, fornecendo $r$ grafos $H_{1}, H_{2}, \ldots, H_{r}$, então, pelo menos um dos grafos $H_{i}(1 \leq i \leq r)$ contém um subgrafo isomorfo a $G_{i}$.

Apesar dos muitos esforços ao longo dos anos, o problema de se determinar o valor dos números de Ramsey está longe de ser resolvido totalmente. Diversos resultados recentes melhoraram os limitantes conhecidos para diversos números de Ramsey de ordem dois (veja, por exemplo, [11, 12, 16, 19, 20]).

Seja $P_{n}$ um caminho com $n \geq 2$ vértices. Um resultado clássico de Gerencsér e Gyárfás [24] afirma que $R\left(P_{n}, P_{n}\right)=\lfloor(3 n-2) / 2\rfloor$. Para alguns grafos particulares, o valor do número de Ramsey é conhecido de forma assintótica. Dada uma árvore $T$, escrevemos $t_{1}$ e $t_{2}$, com $t_{2} \geq t_{1}$, para os tamanhos das classes de vértices de $T$, quando vista como um grafo bipartido. Uma construção simples mostra que $R(T, T) \geq \max \left\{2 t_{1}+t_{2}, 2 t_{2}\right\}-1$. Haxell, Łuczak e Tingley [32] determinaram assintoticamente o valor de $R(T, T)$ para árvores $T$ com $\Delta(T)=o\left(t_{2}\right)$, provando que $R(T, T)=(1+o(1))\left(2 t_{1}+t_{2}\right)$ se $2 t_{1} \geq t_{2}$, e $R(T, T)=(1+o(1)) 2 t_{2}$ se $2 t_{1}<t_{2}$.

Dizemos que um grafo $H=\left(W, E_{H}\right)$ possui largura de banda ("bandwidth") no máximo $b$, se existe uma rotulação dos vértices de $H$ com os números $1, \ldots, n$, de modo que, para cada aresta $i j \in E_{H}$, temos $|i-j| \leq b$. No Capítulo 4, apresentamos uma versão do resultado de Haxell, Łuczak e Tingley para grafos bipartidos com largura de banda pequena e grau 
máximo limitado. Mostramos que, para todo número natural $\Delta$, existe uma constante $\beta>0$ tal que, para todo grafo bipartido $H$ com $n$ vértices, largura de banda no máximo $\beta n$ e grau máximo limitado superiormente por $\Delta$, onde existe uma 2-coloração própria $\chi: V_{H} \rightarrow[2]$, com $t_{1}=\left|\chi^{-1}(1)\right|, t_{2}=\left|\chi^{-1}(2)\right|$ e $t_{1} \leq t_{2}$, temos $R(H, H)=(1+o(1)) \max \left\{2 t_{1}+t_{2}, 2 t_{2}\right\}$.

Para números de Ramsey de ordem três, poucos resultados são conhecidos. Em [30], prova-se que, desde que $n$ seja suficientemente grande, $R\left(P_{n}, P_{n}, P_{n}\right)=2 n-1$ quando $n$ é ímpar, e $R\left(P_{n}, P_{n}, P_{n}\right)=2 n-2$ quando $n$ é par. Esse resultado também foi provado, de forma assintótica, em [18]. Aprimorando técnicas em [29], Benevides e Skokan [3] resolveram completamente o problema para circuitos pares suficientemente grandes, mostrando que $R\left(C_{n}, C_{n}, C_{n}\right)=2 n$ para $n$ suficientemente grande. No Capítulo 4 , estendemos assintoticamente esses resultados para grafos bipartidos com largura de banda pequena e grau máximo limitado, onde as classes do grafo possuem aproximadamente o mesmo tamanho (grafos satisfazendo essa condição são chamados de balanceados). Mostramos que para todo número natural $\Delta$, existe uma constante $\beta>0$ tal que, para todo grafo bipartido balanceado $H$ com $n$ vértices, largura de banda no máximo $\beta n$ e grau máximo limitado superiormente por $\Delta$, temos $R(H, H, H)=(2+o(1)) n$. Como corolário de nossos resultados, determinamos assintoticamente os números de Ramsey de ordem dois e três para grades.

Organizamos os capítulos subsequentes como segue. No Capítulo 2, introduzimos alguns conceitos e fixamos a notação que será utilizada ao longo deste trabalho. No Capítulo 3 , discutimos e provamos o lema de imersão de hipergrafos fixos em hipergrafos pseudoaleatórios. Os resultados sobre números de Ramsey são apresentados no Capítulo 4 e encerramos, no Capítulo 5, com alguns comentários finais sobre os resultados obtidos. 


\section{Capítulo 2}

\section{Preliminares}

Neste capítulo introduzimos alguns conceitos importantes e fixamos a notação básica que será utilizada nos capítulos subsequentes. Denotamos por $[k]$ o conjunto $\{1, \ldots, k\}$. Dadas funções $f, g: \mathbb{N} \rightarrow \mathbb{R}_{+}$e um natural $n$, dizemos que $f(n)=o(g(n))$ se e somente se, para todo $\varepsilon>0$, existe $n_{0}$ tal que, se $n \geq n_{0}$, então $f(n)<\varepsilon g(n)$. Algumas vezes escrevemos $f(n) \ll g(n)$ ao invés de $f(n)=o(g(n))$. A seguir introduzimos, respectivamente, conceitos relacionados a grafos e hipergrafos.

\subsection{Grafos}

Um grafo $G$ é um par de conjuntos finitos $\left(V_{G}, E_{G}\right)$, onde $V_{G}$ é chamado de conjunto de vértices de $G$, e $E_{G}$, o conjunto de arestas de $G$, é um conjunto de pares não ordenados de elementos distintos de $V_{G}$. Escrevemos $e(G)$ e $v(G)$, respectivamente, para representar a cardinalidade de $E(G)$ e $V(G)$. Escrevemos, por simplicidade, $v w$ (ou $w v$ ) para denotar a aresta $\{v, w\} \in E_{G}$, e se $v w \notin E_{G}$, então $v w$ é chamada de não-aresta de $G$. Dizemos que $v w$ incide em $v$ e em $w$ (ou é incidente a $v$ e $w$ ), e $v$ e $w$ são as extremidades da aresta $v w$. Nesse caso, dizemos que os vértices $v$ e $w$ são vizinhos. Se duas arestas possuem uma extremidade comum, então dizemos que essas arestas são adjacentes. Dado um vértice $v \in V_{G}$, definimos o grau de $v$, denotado por $d_{G}(v)$, como a quantidade de vizinhos de $v$, isto é, $d_{G}(v)=\left|\left\{w \in V_{G}: v w \in E_{G}\right\}\right|$. Quando estiver claro a que grafo $G$ estamos nos referindo, escrevemos simplesmente $d(v)$. Por fim, definimos o grau máximo e o grau mínimo de $G$, respectivamente, como $\Delta(G)=\max \left\{d(v): v \in V_{G}\right\}$ e $\delta(G)=\min \left\{d(v): v \in V_{G}\right\}$.

Dado um grafo $G=\left(V_{G}, E_{G}\right)$ com $n$ vértices e dois subconjuntos de vértices $A$ e $B$, não vazios e disjuntos, denotamos por $E_{G}(A, B)$ o subconjunto de arestas de $E_{G}$ que possuem uma extremidade em $A$ e outra em $B$. Ademais, a cardinalidade de $E_{G}(A, B)$ é denotada por $e_{G}(A, B)$. A densidade de $G$ é dada por $d_{G}=e(G) /\left(\begin{array}{l}n \\ 2\end{array}\right)$ e dizemos que

$$
d_{G}(A, B)=\frac{e_{G}(A, B)}{|A||B|}
$$


é a densidade de $G$ entre $A$ e $B$.

Se $G$ é um grafo tal que $V_{G}$ pode ser dividido em dois subconjuntos disjuntos $A$ e $B$ e te$\operatorname{mos} E_{G}=E_{G}(A, B)$, então dizemos que $G$ é um grafo bipartido e escrevemos $G=\left(A, B ; E_{G}\right)$. Um grafo com $n$ vértices que possui todas as $\left(\begin{array}{l}n \\ 2\end{array}\right)$ arestas possíveis é chamado de grafo completo ou clique e é denotado por $K_{n}$.

Um grafo $H$ é dito subgrafo de um grafo $G$, e escrevemos $H \subset G$, se $V_{H} \subset V_{G}$ e $E_{H} \subset E_{G}$. Ademais, se $V_{H}=V_{G}$, então dizemos que $H$ é um subgrafo gerador de $G$. Se um grafo $G$ não contém nenhum subgrafo isomorfo a $H$, dizemos que $G$ é livre de $H$. Dado um subconjunto de vértices $W \subset V_{G}$, denotamos por $G[W]$ o grafo com conjunto de vértices $W$ tal que o conjunto de arestas de $G[W]$ é composto pelo subconjunto de arestas de $G$ que possuem as duas extremidades em $W$. Dizemos que o grafo $G[W]$ é o subgrafo de $G$ induzido por $W$.

Um grafo $P$ com conjunto de vértices $V_{P}=\left\{v_{1}, \ldots, v_{k}\right\}$ é chamado de caminho se $E_{P}=\left\{v_{1} v_{2}, v_{2} v_{3}, \ldots, v_{k-1} v_{k}\right\}$. Se um grafo $P^{\prime}$ possui conjunto de vértices $V_{P^{\prime}}=\left\{v_{1}, \ldots, v_{k}\right\}$ e conjunto de arestas dado por $E_{P} \cup\left\{v_{k} v_{1}\right\}$, então $P^{\prime}$ é chamado de circuito. Um grafo $G$ é conexo se existe um caminho entre qualquer par de vértices $\{v, w\}$. Ademais, se $T$ é um grafo conexo e não possui circuitos, então dizemos que $T$ é uma árvore. Por fim, dado um grafo $G$, um emparelhamento $M$ em $G$ é um conjunto de arestas de $G$ duas a duas não adjacentes.

Uma partição $\mathcal{P}=\left\{V_{1}, \ldots, V_{k}\right\}$ dos vértices de um grafo $G$ é uma família de conjuntos $V_{i} \subset V_{G}$, para $1 \leq i \leq k$, tal que esses conjuntos são disjuntos dois a dois e $V_{1} \cup \ldots \cup V_{k}=V_{G}$. Dizemos que cada $V_{i}$ é uma classe da partição $\mathcal{P}$.

Dado um grafo $G$, uma $k$-coloração dos vértices de $G$ é uma função $\chi_{V}: V_{G} \rightarrow\{1, \ldots, k\}$, isto é, uma função que atribui uma 'cor' para cada vértice de $G$, dentre $k$ cores disponíveis. Analogamente, uma $k$-coloração das arestas de $G$ é uma função $\chi_{E}: E_{G} \rightarrow\{1, \ldots, k\}$. Se $\chi_{V}$ atribui cores distintas para todo par de vértices $\{v, w\}$ tal que $u$ é vizinho de $w$, então $\chi_{V}$ é dita uma coloração própria dos vértices de $G$. Analogamente, se arestas adjacentes recebem cores distintas da coloração $\chi_{E}$, então $\chi_{E}$ é dita uma coloração própria das arestas de $G$. Se, dada uma coloração das arestas de um grafo $G$, todas as arestas de um subgrafo $H \subset G$ possuem a mesma cor, então dizemos que $H$ é monocromático.

\subsection{Hipergrafos}

Um hipergrafo $H$ é um par de conjuntos finitos $\left(V_{H}, E_{H}\right)$, onde $V_{H}$ é o conjunto de vértices de $H$ e o conjunto de hiperarestas $E_{H}$ é uma coleção de subconjuntos de vértices. Por simplicidade, referimo-nos às hiperarestas simplesmente por 'arestas'. Se todas as arestas de $E_{H}$ são subconjuntos com $k$ vértices, dizemos que $H$ é um hipergrafo $k$-uniforme. Note que um grafo é um hipergrafo 2-uniforme. Um hipergrafo $H$ é dito linear se cada par de arestas compartilha no máximo um vértice. Nesta seção introduzimos alguns conceitos que são utilizados no Capítulo 3.

Denotamos por $\left(\begin{array}{c}V_{H} \\ i\end{array}\right)$ o conjunto de todos os subconjuntos de $V_{H}$ com tamanho $i$ e de- 
notamos por $V_{H}^{i}$ o subconjunto de todas as sequências de $V_{H}$ com $i$ elementos, isto é, cada elemento de $V_{H}^{i}$ é composto por $i$ vértices de $H$, de modo que a ordem desses $i$ vértices é relevante.

As seguintes definições estão relacionadas com a noção de vizinhança em hipergrafos $k$-uniformes. Para $1 \leq i \leq k-1$, dado $\left\{x_{1}, \ldots, x_{i}\right\} \in\left(\begin{array}{c}V_{H} \\ i\end{array}\right)$, defina

$$
N_{H}\left(x_{1}, \ldots, x_{i}\right)=\left\{\left\{x_{i+1}, \ldots, x_{k}\right\} \in\left(\begin{array}{c}
V_{H} \\
k-i
\end{array}\right):\left\{x_{1}, \ldots, x_{k}\right\} \in E_{H}\right\},
$$

i.e., $N_{H}\left(x_{1}, \ldots, x_{i}\right)$ é o conjunto de elementos de $\left(\begin{array}{c}V_{H} \\ k-i\end{array}\right)$ que, juntamente com $\left\{x_{1}, \ldots, x_{i}\right\}$, formam uma aresta de $H$. Defina $d_{H}\left(x_{1}, \ldots, x_{i}\right)=\left|N_{H}\left(x_{1}, \ldots, x_{i}\right)\right|$ como sendo o grau de $\left\{x_{1}, \ldots, x_{i}\right\}$. Em particular, se $x \in V_{H}$, então

$$
N_{H}(x)=\left\{\left\{x_{1}, \ldots, x_{k-1}\right\} \in\left(\begin{array}{c}
V_{H} \\
k-1
\end{array}\right):\left\{x, x_{1}, \ldots, x_{k-1}\right\} \in E_{H}\right\} .
$$

Ademais, seja $N_{H}^{\text {con }}(x)=\left\{y: \exists\left\{x_{1}, \ldots, x_{k-1}\right\} \in N_{H}(x)\right.$ com $\left.y \in\left\{x_{1}, \ldots, x_{k-1}\right\}\right\}$ o conjunto dos vértices de $H$ que pertencem a alguma aresta que contém $x$. Dada uma coleção $X \subset\left(\begin{array}{c}V_{H} \\ k-1\end{array}\right)$ de conjuntos com $k-1$ vértices, defina

$$
N_{H}(X)=\left\{x: x \in N_{H}\left(x_{1}, \ldots, x_{k-1}\right) \text { para todo }\left\{x_{1}, \ldots, x_{k-1}\right\} \in X\right\}
$$

i.e., $N_{H}(X)$ é a vizinhança comum dos elementos de $X$.

Finalizando, uma imersão de um hipergrafo $H$ em um hipergrafo $G$ é um mapeamento injetivo $\phi: V_{H} \rightarrow V_{G}$ tal que $\phi\left(v_{1}\right), \ldots, \phi\left(v_{k}\right) \in E_{G}$ sempre que $v_{1}, \ldots, v_{k} \in E_{H}$. 


\section{Capítulo 3}

\section{Lema de contagem para hipergrafos pseudoaleatórios}

Existem várias noções de pseudoaleatoriedade para hipergrafos 2-uniformes (grafos) na literatura. Algumas tratam da uniformidade na distribuição das arestas do grafo e outras, por exemplo, são baseadas no valor do segundo maior autovalor (em valor absoluto) da matriz de adjacências do grafo. Uma noção também importante de pseudoaleatoriedade em grafos é relacionada com a quantidade de circuitos de tamanho 4 que o grafo possui (veja [10, 19, 8, 44, 54]).

Os conceitos de pseudoaleatoriedade citados no parágrafo anterior levam em conta características globais do grafo. No entanto, algumas noções possuem um caráter mais local, sendo relacionadas ao grau dos vértices e ao cograu dos pares de vértices. Em [22], esse conceito é generalizado para hipergrafos uniformes. Por simplicidade, consideremos, por ora, apenas hipergrafos 3-uniformes. Sejam $v_{1}, \ldots, v_{t}$ vértices de um hipergrafo 3 -uniforme $G$ e seja $J$ um grafo com conjunto de vértices $[t]$. Denotamos por $d_{J, G}\left(v_{1}, \ldots, v_{t}\right)$ a quantidade de vértices $x \in G$ tais que $\left\{v_{i}, v_{j}, x\right\} \in E(G)$ para toda aresta $\{i, j\} \in E(J)$. Em [22], uma noção de pseudoaleatoriedade é dada pela definição abaixo.

Definição 3.1. Um hipergrafo 3-uniforme $G$ é dito $(\varepsilon, p)$-uniforme se, para todo grafo auxiliar $J$ com $t \leq 7$ vértices e $s \leq 6$ arestas, e para toda escolha de vértices distintos $v_{1}, \ldots, v_{t} \in V_{G}$ temos

$$
\left|d_{J, G}\left(v_{1}, \ldots, v_{t}\right)-n p^{s}\right| \leq \varepsilon n p^{s} .
$$

A condição apresentada na definição acima é um pouco forte. De fato, no mesmo trabalho em que a condição acima é apresentada (veja [22]), uma definição de pseudoaleatoriedade necessita do controle de somente 5 grafos auxiliares, a saber:

$J_{1}$ : grafo com dois vértices e uma aresta;

$J_{2}$ : grafo com quatro vértices e duas arestas não adjacentes;

$J_{3}$ : grafo com três vértices e duas arestas; 
$J_{4}$ : grafo com quatro vértices e três arestas formando um caminho;

$J_{5}$ : grafo com sete vértices e seis arestas formando dois caminhos de mesmo tamanho que se interceptam em exatamente um vértice interno de cada caminho.

Assim, consideremos a seguinte definição de hipergrafos $(\varepsilon, p)$-uniformes utilizada em [22].

Definição 3.2. Um hipergrafo 3-uniforme $G$ é dito $(\varepsilon, p)$-uniforme se para $i=1, \ldots, 5$, e para toda escolha de vértices distintos $v_{1}, \ldots, v_{\left|V\left(J_{i}\right)\right|} \in V_{G}$ temos

$$
\left|d_{J, G}\left(v_{1}, \ldots, v_{\left|V\left(J_{i}\right)\right|}\right)-n p^{\left|E\left(J_{i}\right)\right|}\right| \leq \varepsilon n p^{\left|E\left(J_{i}\right)\right|} .
$$

Definimos agora algumas propriedades de hipergrafos que dizem respeito à vizinhança comum de alguns subconjuntos de vértices. Com essas propriedades, vamos definir o conceito de pseudoaleatoriedade que iremos considerar neste capítulo. No que segue, tome $k \geq 2$.

Propriedade 3.3 (LMT - Propriedade do limitante). Definimos $\operatorname{LMT}^{k}(C, t)$ como a familia de hipergrafos $k$-uniformes $G$ com $\left|V_{G}\right|=n$ e $p=|E(G)| /\left(\begin{array}{l}n \\ k\end{array}\right)$ tais que, para todo $1 \leq r \leq t$ e todos subconjuntos distintos $S_{1}, \ldots, S_{r} \in\left(\begin{array}{c}V_{G} \\ k-1\end{array}\right)$, temos

$$
\left|N_{G}\left(S_{1}\right) \cap \ldots \cap N_{G}\left(S_{r}\right)\right| \leq C n p^{r}
$$

Propriedade 3.4 (CG - Propriedade do cograu). Definimos $\mathrm{CG}_{\varepsilon}^{k}(t)$ como a familia de hipergrafos $k$-uniformes $G$ com $\left|V_{G}\right|=n$ e $p=|E(G)| /\left(\begin{array}{l}n \\ k\end{array}\right)$ tal que, para todo $1 \leq r \leq t$, temos que

$$
|| N_{G}\left(S_{1}\right) \cap \ldots \cap N_{G}\left(S_{r}\right)\left|-n p^{r}\right|<\varepsilon n p^{r}
$$

para mais que $(1-\varepsilon)\left(\begin{array}{c}\left(\begin{array}{c}n \\ k-1\end{array}\right) \\ r\end{array}\right)$ famílias $\left\{S_{1}, \ldots, S_{r}\right\}$ de subconjuntos distintos de $\left(\begin{array}{c}V_{G} \\ k-1\end{array}\right)$.

Se um hipergrafo $k$-uniforme $G$ pertence a $\operatorname{LMT}^{k}\left(C, t_{1}\right)$ e $\mathrm{CG}_{\varepsilon}^{k}\left(t_{2}\right)$, dizemos que $G$ é um hipergrafo $\left(C, t_{1}, t_{2}, \varepsilon\right)$-pseudoaleatório. O lema de contagem que será apresentado nesta seção diz respeito à imersão de hipergrafos $k$-uniformes em hipergrafos $G$ que contêm propriedades pseudoaleatórias mais fracas que as apresentadas na Definição 3.2, a saber, consideramos hipergrafos $G$ que são $(C, t, 2, \delta)$-pseudoaleatórios. Por exemplo, para hipergrafos 3-uniformes $(C, t, 2, \delta)$-pseudoaleatórios, precisamos ter controle total somente sobre os grafos auxiliares $J_{1}, J_{2}$ e $J_{3}$. Ademais, esse controle não precisa ser para toda escolha de pares de vértices distintos em $G$, e sim para a maioria dos pares, como pode ser visto na definição da propriedade $\mathrm{CG}_{\varepsilon}^{k}(2)$. Uma noção de pseudoaleatoriedade semelhante à que consideramos neste capítulo é utilizada em [21].

Dado um hipergrafo linear $k$-uniforme $H$, um conector é uma aresta $e \in E_{H}$ tal que existem $x \in V_{H} \backslash\{e\}$ e $k$ arestas $e_{1}, \ldots, e_{k}$ que contêm $x$, onde $\left|e \cap e_{i}\right|=1$ para todo $i=1, \ldots, k$. Dizemos que um hipergrafo $H$ é livre de conectores se não possui conectores. Ademais, note que os grafos (hipergrafos 2-uniformes) livres de conectores são os grafos livres 
de triângulos. Defina $d_{H}=\max \{\delta(J): J \subset H\}$ e $D_{H}=\min \left\{k d_{H}, \Delta(H)\right\}$, onde $\delta(H)$ e $\Delta(H)$ são, respectivamente, os graus mínimo e máximo de $H$. Kohayakawa, Rödl e Sissokho [37] provaram que, dado um grafo fixo $H$ livre de triângulos e $p \gg n^{-1 / D_{H}} \operatorname{com} p=o(1)$, para todo $\varepsilon>0$, existem $\delta>0$ e um natural $n_{0}$ tal que, se $n \geq n_{0}$ e $G$ é um grafo com $n$ vértices, onde $|E(G)|=\left(\begin{array}{l}n \\ 2\end{array}\right) p, G \in \operatorname{LMT}^{2}\left(C, D_{H}\right)$ para algum $C>1$ e $G \in \mathrm{CG}_{\delta}^{2}(2)$, então

$$
|| \mathcal{I}(H, G)\left|-n^{v(H)} p^{e(H)}\right|<\varepsilon n^{v(H)} p^{e(H)},
$$

onde $\mathcal{I}(H, G)$ é o conjunto de todas as imersões de $H$ em $G$. O resultado principal deste capítulo, Teorema 3.5 , estende esse resultado para hipergrafos lineares $k$-uniformes livre de conectores.

Teorema 3.5. Sejam $C>1, m \geq 4$ inteiros e considere um hipergrafo linear $k$-uniforme $H$ com $m$ vértices e livre de conectores. Se $p=p(n)=o(1)$ tal que $p \gg n^{-1 / D_{H}}$, então, para todo $\varepsilon>0$, existe $\delta>0$ e um inteiro $n_{0}>0$ tal que o seguinte vale. Se uma sequência de hipergrafos k-uniformes $\left\{G_{n}\right\}_{n=1}^{\infty}$ com $\left|V\left(G_{n}\right)\right|=n$ é tal que, para todo $n$, o hipergrafo $G_{n}$ é $\left(C, D_{H}, 2, \delta\right)$-pseudoaleatório e $p=p(n)=e\left(G_{n}\right) /\left(\begin{array}{l}n \\ k\end{array}\right)$, então, para todo $n \geq n_{0}$,

$$
|| \mathcal{I}\left(H, G_{n}\right)\left|-n^{m} p^{e(H)}\right|<\varepsilon n^{m} p^{e(H)}
$$

Na Seção 3.1 enunciamos dois lemas que, juntos, compõem a prova do Teorema 3.5. A Seção 3.2 contém alguns resultados que são necessários para a prova do Lema 3.6, apresentado na Seção 3.1. Na Seção 3.3 apresentamos o Lema de Extensão, um importante resultado, útil para a prova do outro lema (Lema 3.7) apresentado na Seção 3.1. Finalmente, na Seção 3.4 . provamos os Lemas 3.6 e 3.7

\subsection{Lemas principais}

Os próximos dois lemas, juntos, compõem a prova do Teorema 3.5

Lema 3.6 (Lema $\mathrm{CG}_{2 \rightarrow t}$ ). Sejam $t \geq 2$ e $C>1$. Suponha que $p=p(n)$ satisfaz $p \gg n^{-1 / t}$. Seja $\left\{G_{n}\right\}_{n=1}^{\infty}$ uma sequência de hipergrafos $k$-uniformes com $G_{n} \in \operatorname{LMT}^{k}(C, 2)$ e $\left|E\left(G_{n}\right)\right|=$ $\left(\begin{array}{l}n \\ k\end{array}\right)$ p para todo $n$. Então, temos o seguinte:

Para todo $\delta_{1}>0$, existem $\delta_{2}>0$ e $n_{1}>0$ tais que, se $n \geq n_{1}$ e $G_{n} \in \mathrm{CG}_{\delta_{2}}^{k}(2)$, então $G_{n} \in \mathrm{CG}_{\delta_{1}}^{k}(t)$.

O próximo resultado é muito similar ao Teorema 3.5. Trocamos apenas a propriedade $\mathrm{CG}_{\delta}^{k}(2)$ por $\mathrm{CG}_{\delta}^{k}\left(d_{H}\right)$.

Lema 3.7 (Lema de contagem modificado). Sejam $C>1, m \geq 4$ e considere um hipergrafo linear $k$-uniforme $H$ com $m$ vértices e livre de conectores. Se $p=p(n)=o(1)$ tal que $p \gg n^{-1 / D_{H}}$, então para todo $\varepsilon>0$ existe $\delta>0$ e um inteiro $n_{2}>0$ tal que o seguinte vale: 
Se uma sequência de hipergrafos k-uniformes $\left\{G_{n}\right\}_{n=1}^{\infty}$ com $\left|V\left(G_{n}\right)\right|=n$ é tal que, para todo $n$, o hipergrafo $G_{n}$ é $\left(C, D_{H}, d_{H}, \delta\right)$-pseudoaleatório e $p=p(n)=e\left(G_{n}\right) /\left(\begin{array}{l}n \\ k\end{array}\right)$, então, para todo $n \geq n_{2}$,

$$
|| \mathcal{I}\left(H, G_{n}\right)\left|-n^{m} p^{e(H)}\right|<\varepsilon n^{m} p^{e(H)} .
$$

Apresentamos agora a prova do Teorema 3.5, utilizando os Lemas 3.6 e 3.7, que são provados na Seção 3.4 .

Prova do Teorema 3.5. Fixe $C>1$ e $m \geq 4$. Seja $H$ um hipergrafo linear $k$-uniforme livre de conectores com $\left|V_{H}\right|=m$. Tome $p=p(n)=o(1)$ tal que $p \gg n^{-1 / D_{H}}$ e fixe $\varepsilon>0$.

Agora fazemos uso dos Lemas 3.6 e 3.7. Uma aplicação do Lema 3.7 com parâmetros $m$, $\varepsilon$ e $C$ nos retorna uma constante $\delta_{1}>0$ e um inteiro $n_{2}>0$. Aplicando o Lema 3.6 com parâmetros $t=d_{H}, C$ e $\delta_{1}$, recebemos uma constante $\delta_{2}>0$ e um inteiro $n_{1}>0$. Tome $n_{0}=\max \left\{n_{1}, n_{2}\right\}$ e considere $n \geq n_{0}$.

Seja $G_{n}$ um hipergrafo $k$-uniforme $G_{n}$ com $n$ vértices e $\left|E\left(G_{n}\right)\right|=\left(\begin{array}{l}n \\ k\end{array}\right) p$. Suponha que $G_{n}$ é $\left(C, D_{H}, 2, \delta_{2}\right)$-pseudoaleatório, i.e., $G_{n} \in \operatorname{LMT}^{k}\left(C, D_{H}\right)$ e $G_{n} \in \mathrm{CG}_{\delta_{2}}^{k}(2)$. Note que, desta forma, também temos que $G_{n} \in \operatorname{LMT}^{k}(C, 2)$. Ademais, $p \gg n^{-1 / D_{H}} \gg n^{-1 / d_{H}}$. Assim, o Lema 3.6 garante que $G_{n} \in \mathrm{CG}_{\delta_{1}}^{k}\left(d_{H}\right)$. Portanto, pelo Lema 3.7, concluímos que

$$
|| \mathcal{I}\left(H, G_{n}\right)\left|-n^{v(H)} p^{e(H)}\right|<\varepsilon n^{v(H)} p^{e(H)} .
$$

\subsection{Resultados auxiliares}

Nesta seção apresentamos alguns resultados que são necessários para provar o Lema 3.6. Na Seção 3.2.1, introduzimos algumas desigualdades combinatórias e, na Seção 3.2.2, generalizamos as propriedades LMT e CG para vértices, em vez de conjuntos de vértices de tamanho $k-1$.

\subsubsection{Alguns resultados combinatórios}

Primeiramente, introduzimos o seguinte lema, cuja prova pode ser vista em [37].

Lema 3.8. Para todo $\delta>0$, existe $\gamma>0$ tal que, se uma família de números reais $a_{i} \geq 0$, para $1 \leq i \leq N$, satisfaz as condições

(i) $\sum_{i=1}^{N} a_{i} \geq(1-\gamma) N a$,

(ii) $\sum_{i=1}^{N} a_{i}^{2} \leq(1+\gamma) N a^{2}$,

então

$$
\left|\left\{i:\left|a_{i}-a\right|<\delta a\right\}\right|>(1-\delta) N .
$$


Os seguintes lemas apresentam algumas desigualdades combinatórias que serão utilizadas com frequência.

Lema 3.9. Para todos $\varepsilon>0, r \geq 1$ e $0<\alpha<1$, existe $n_{0}$ tal que, se $n \geq n_{0}$, então

$$
\left(\begin{array}{c}
n \alpha \\
r
\end{array}\right) \geq(1-\varepsilon) \alpha^{r}\left(\begin{array}{l}
n \\
r
\end{array}\right)
$$

Demonstração. Fixe $\varepsilon>0, r \geq 1$ e $0<\alpha<1$. Tome $\delta=1-(1-\varepsilon)^{1 / r}$ e considere $n \geq n_{0}$, onde $n_{0}=\lceil(r-1)(1+(1-\alpha) / \alpha \delta)\rceil$. Note que, para todo $0 \leq k \leq r-1$, as escolhas de $n_{0}$ e $\delta$ implicam que

$$
n \alpha-k=\left(1-\frac{(1-\alpha) k}{\alpha(n-k)}\right)(n-k) \alpha \geq(1-\delta)(n-k) \alpha=(1-\varepsilon)^{1 / r}(n-k) \alpha .
$$

Portanto,

$$
\left(\begin{array}{c}
n \alpha \\
r
\end{array}\right)=\prod_{k=0}^{r-1} \frac{n \alpha-k}{r-k} \geq(1-\varepsilon) \alpha^{r} \prod_{k=0}^{r-1} \frac{n-k}{r-k}=(1-\varepsilon) \alpha^{r}\left(\begin{array}{l}
n \\
r
\end{array}\right) .
$$

Lema 3.10. Para todos $\varepsilon>0, r \geq 1$ e $b>0$, existe $n_{0}$ tal que, se $n \geq n_{0}$, então

$$
\left(\begin{array}{c}
n b \\
r
\end{array}\right) \leq(1+\varepsilon) b^{r}\left(\begin{array}{l}
n \\
r
\end{array}\right)
$$

Demonstração. Fixe $\varepsilon>0, r \geq 1$ e $b>0$. Agora fixe $\delta=(1+\varepsilon)^{1 / r}-1$ e considere $n \geq n_{0}$, onde $n_{0}=\lceil(r-1)(1+(b-1) / b \delta)\rceil$. Note que, para todo $0 \leq k \leq r-1$, se $b \leq 1$, temos

$$
n b-k \leq(1+\varepsilon)^{1 / r}(n-k) b .
$$

Por outro lado, se $b>1$, as escolhas de $n_{0}$ e $\delta$ implicam que

$$
n b-k=\left(1+\frac{(b-1) k}{b(n-k)}\right)(n-k) b \leq(1+\delta)(n-k) b=(1+\varepsilon)^{1 / r}(n-k) b
$$

Portanto,

$$
\left(\begin{array}{c}
n b \\
r
\end{array}\right)=\prod_{k=0}^{r-1} \frac{n b-k}{r-k} \leq(1+\varepsilon) b^{r} \prod_{k=0}^{r-1} \frac{n-k}{r-k}=(1+\varepsilon) b^{r}\left(\begin{array}{c}
n \\
r
\end{array}\right) .
$$

\subsubsection{Generalizando as propriedades LMT e CG}

Considere constantes $C>1$ e $\beta>0$. Suponha que um hipergrafo $k$-uniforme $G_{n}$ com $n$ vértices é $(C, 2,2, \beta)$-pseudoaleatório. Para referências futuras, enunciamos abaixo as consequências desse fato. 
- Para todo $S \in\left(\begin{array}{c}V_{G} \\ k-1\end{array}\right)$ :

$$
|N(S)| \leq C n p
$$

- Para todos conjuntos distintos $S_{1}, S_{2} \in\left(\begin{array}{c}V_{G} \\ k-1\end{array}\right)$ :

$$
\left|N\left(S_{1}\right) \cap N\left(S_{2}\right)\right| \leq C n p^{2}
$$

- Para mais que $(1-\beta)\left(\begin{array}{c}n \\ k-1\end{array}\right)$ conjuntos $S \in\left(\begin{array}{c}V_{G} \\ k-1\end{array}\right)$ :

$$
|| N(S)|-n p|<\beta n p
$$

- Para mais que $\left.(1-\beta)\left(\begin{array}{c}n \\ k-1 \\ 2\end{array}\right)\right)$ conjuntos distintos $S_{1}, S_{2} \in\left(\begin{array}{c}V_{G} \\ k-1\end{array}\right)$ :

$$
|| N\left(S_{1}\right) \cap N\left(S_{2}\right)\left|-n p^{2}\right|<\beta n p^{2} ;
$$

Se um hipergrafo $k$-uniforme $G$ com $n$ vértices pertence a $\operatorname{LMT}^{k}(C, 2)$, então não existe um conjunto com $k-1$ vértices que possui vizinhança 'grande', e pares de conjuntos distintos com $k-1$ vértices também não possuem vizinhança comum 'grande'. O próximo resultado afirma que, se $G$ pertence a $\operatorname{LMT}^{k}(C, 2)$, então isto é verdade não somente para conjuntos de $k-1$ vértices, mas para qualquer conjunto com $i$ vértices, onde $1 \leq i \leq k-1$. Particularmente, o caso $i=1$ é importante para nossa prova. Isso pode ser visto como uma versão de $\operatorname{LMT}^{k}(C, 2)$ para vértices, em vez de conjuntos com $k-1$ vértices.

Lema 3.11. Seja $G$ um hipergrafo k-uniforme com $n$ vértices. Se $G$ satisfaz $\operatorname{LMT}^{k}(C, 2)$, então, para cada $1 \leq i \leq k-1$, vale $\left|N\left(S_{1}\right)\right| \leq C n^{k-i} p$ e $\left|N\left(S_{1}\right) \cap N\left(S_{2}\right)\right| \leq C n^{k-i} p^{2}$ para todos os subconjuntos distintos $S_{1}, S_{2} \in\left(\begin{array}{c}V_{G} \\ i\end{array}\right)$. Em particular,

(i) $d(u) \leq C n^{k-1} p$, para todo $u \in V_{G}$,

(ii) $|N(u) \cap N(v)| \leq C n^{k-1} p^{2}$, para todos $\{u, v\} \in\left(\begin{array}{c}V_{G} \\ 2\end{array}\right)$.

Demonstração. Seja $G$ um hipergrafo $k$-uniforme com $n$ vértices e suponha $G \in \operatorname{LMT}^{k}(C, 2)$. A prova segue por indução em $i$.

Pela definição de $\operatorname{LMT}^{k}(C, 2)$, o resultado é válido para $i=k-1$. Agora assuma que as condições (i) e (ii) são válidas para $1<i<k-1$. Vamos mostrar que o resultado é válido para $i-1$.

Fixe conjuntos distintos $S_{1}, S_{2} \in\left(\begin{array}{c}V_{G} \\ i-1\end{array}\right)$. Começamos provando que $\left|N\left(S_{1}\right)\right| \leq C n^{k-(i-1)} p$. Sabemos que $\left|N\left(S_{1}\right)\right|=\sum_{u \in V_{G} \backslash S_{1}}\left|N\left(S_{1} \cup u\right)\right|$. Portanto, utilizando a hipótese indutiva, temos que $\left|N\left(S_{1}\right)\right| \leq n\left(C n^{k-i} p\right)=C n^{k-(i-1)} p$. Para verificar a outra condição, utilizamos a mesma ideia. Como $\left|N\left(S_{1}\right) \cap N\left(S_{2}\right)\right|=\sum_{u \in V_{G} \backslash\left(S_{1} \cup S_{2}\right)}\left|N\left(S_{1} \cup u\right) \cap N\left(S_{2} \cup u\right)\right|$, a hipótese indutiva garante que $\left|N\left(S_{1}\right) \cap N\left(S_{2}\right)\right| \leq n\left(C n^{k-i} p^{2}\right)=C n^{k-(i-1)} p^{2}$. 
O próximo lema afirma que, se $G$ é um hipergrafo $(C, 2,2, \beta)$-pseudoaleatório $k$-uniforme com $n$ vértices, então a maioria dos vértices de $G$ possui grau 'próximo' de $n p$, e para a maioria dos pares de vértices de $G$, a quantidade de vizinhos em comum é 'próxima' de $n p^{2}$. Isso pode ser visto como uma versão de $\mathrm{CG}_{\beta}^{k}(2)$ para vértices, em vez de conjuntos com $k-1$ vértices.

Lema 3.12. Seja $C>1$ uma constante fixa e suponha que $p=p(n)$ satisfaz $p \gg n^{-(k-1) / 2}$. Se $\left\{G_{n}\right\}_{n=1}^{\infty}$ é uma sequência de hipergrafos $k$-uniformes com $G_{n} \in \operatorname{LMT}^{k}(C, 2)$ para todo $n$, onde $\left|E\left(G_{n}\right)\right|=\left(\begin{array}{l}n \\ k\end{array}\right)$ p, então o seguinte é válido para $\left\{G_{n}\right\}_{n=1}^{\infty}$ :

Para todo $\delta>0$, existem $\beta>0$ e $n_{0}>0$ tais que, se $n \geq n_{0}$ e $G_{n} \in \mathrm{CG}_{\beta}^{k}(2)$, então

(i) $\left|d(u)-\left(\begin{array}{c}n \\ k-1\end{array}\right) p\right|<\delta\left(\begin{array}{c}n \\ k-1\end{array}\right)$ p para mais que $(1-\delta) n$ vértices $u \in V_{G}$;

(ii) ||$N(u) \cap N(v)\left|-\left(\begin{array}{c}n \\ k-1\end{array}\right) p^{2}\right|<\delta\left(\begin{array}{c}n \\ k-1\end{array}\right) p^{2}$ para mais que $(1-\delta)\left(\begin{array}{c}n \\ 2\end{array}\right)$ pares $\{u, v\} \in\left(\begin{array}{c}V_{G} \\ 2\end{array}\right)$.

Demonstração. As provas dos itens (i) e (ii) são similares, mas, por completude, apresentamos as duas provas. Fixe $C>1$ e suponha que $p \gg n^{-(k-1) / 2}$. Fixe $\delta>0$ e seja $\gamma>0$ obtido por uma aplicação do Lema 3.8 com parâmetro $\delta>0$. Tome

$$
\beta=\min \left\{1-(1-\gamma)^{1 / 4},(1+\gamma / 2)^{1 / 3}-1, \gamma / 2\left(1+2 C^{2}\right)\right\}
$$

Pela suposição sobre $p$, sabemos que, para $n$ suficientemente grande, temos

$$
p \geq\left(\beta^{-1}(1+\beta) C^{2}(k-1)^{k-1}\right) n^{-(k-1) / 2} .
$$

No que segue supomos $n \geq n_{0}$, onde $n_{0}$ é uma constante suficientemente grande, de modo que a desigualdade acima é satisfeita. Considere um hipergrafo $k$-uniforme $G=G_{n}$ com $n$ vértices e $\left|E\left(G_{n}\right)\right|=\left(\begin{array}{l}n \\ k\end{array}\right) p$. Suponha que $G_{n} \in \mathrm{LMT}^{k}(C, 2)$ e $G_{n} \in \mathrm{CG}_{\beta}^{k}(2)$.

Começamos provando o item (i). Vamos mostrar que as duas condições do Lema 3.8 são satisfeitas. As equações (3.5) e 3.10) a seguir podem ser vistas como as condições requeridas pelo Lema 3.8 .

Primeiramente, note que

$$
\begin{aligned}
\sum_{u \in V_{G}} d(u) & =\sum_{S \in\left(\begin{array}{c}
V_{G} \\
k-1
\end{array}\right)}|N(S)| \\
& \geq \sum_{S \in\left(\begin{array}{c}
V_{G} \\
k-1
\end{array}\right):|N(S)| \geq(1-\beta) n p}|N(S)| \\
& \geq(1-\beta)^{2}\left(\begin{array}{c}
n \\
k-1
\end{array}\right) n p \\
& \geq(1-\gamma) n\left(\begin{array}{c}
n \\
k-1
\end{array}\right) p
\end{aligned}
$$

onde a primeira desigualdade é trivial, a segunda segue de (3.3) e a última é consequência da escolha de $\beta$. Assim, a primeira condição do Lema 3.8 é satisfeita. Para a segunda, observe 
que

$$
\begin{aligned}
& \sum_{u \in V_{G}} d(u)^{2}=\sum_{S_{1}, S_{2} \in\left(\begin{array}{c}
V_{G} \\
k-1
\end{array}\right)}\left|N\left(S_{1}\right) \cap N\left(S_{2}\right)\right| \\
& =2 \sum_{\left\{S_{1}, S_{2}\right\} \in\left(\begin{array}{c}
V_{G} \\
k-1 \\
2
\end{array}\right)}\left|N\left(S_{1}\right) \cap N\left(S_{2}\right)\right|+\sum_{S \in\left(\begin{array}{c}
V_{G} \\
k-1
\end{array}\right)}|N(S)| .
\end{aligned}
$$

Limitaremos os dois termos da igualdade acima. Por (3.1), temos

$$
\sum_{S \in\left(\begin{array}{c}
V_{G} \\
k-1
\end{array}\right)}|N(S)| \leq\left(\begin{array}{c}
n \\
k-1
\end{array}\right) C n p
$$

Como $p \geq\left((k-1)^{k-1} C / \beta\right) n^{-(k-1)}$, podemos concluir que $C \leq \beta\left(\begin{array}{c}n \\ k-1\end{array}\right) p$. Portanto,

$$
\sum_{S \in\left(\begin{array}{c}
V_{G} \\
k-1
\end{array}\right)}|N(S)| \leq \beta n\left(\left(\begin{array}{c}
n \\
k-1
\end{array}\right) p\right)^{2}
$$

Agora definimos $A=\left\{\left\{S_{1}, S_{2}\right\} \in\left(\begin{array}{c}\left(\begin{array}{c}V_{G} \\ k-1 \\ 2\end{array}\right) \\ 2\end{array}\right):\left|N\left(S_{1}\right) \cap N\left(S_{2}\right)\right| \leq(1+\beta) n p^{2}\right\}$ e, por fim, $B=\left\{\left\{S_{1}, S_{2}\right\} \in\left(\begin{array}{c}\left(\begin{array}{c}V_{G} \\ k-1\end{array}\right) \\ 2\end{array}\right):\left|N\left(S_{1}\right) \cap N\left(S_{2}\right)\right|>(1+\beta) n p^{2}\right\}$. Note que

$$
\sum_{\left\{S_{1}, S_{2}\right\} \in\left(\left(\begin{array}{c}
V_{G} \\
k-1 \\
2
\end{array}\right)\right.}\left|N\left(S_{1}\right) \cap N\left(S_{2}\right)\right|=\sum_{\left\{S_{1}, S_{2}\right\} \in A}\left|N\left(S_{1}\right) \cap N\left(S_{2}\right)\right|+\sum_{\left\{S_{1}, S_{2}\right\} \in B}\left|N\left(S_{1}\right) \cap N\left(S_{2}\right)\right|
$$

Mas, claramente,

$$
\sum_{\left\{S_{1}, S_{2}\right\} \in A}\left|N\left(S_{1}\right) \cap N\left(S_{2}\right)\right| \leq \frac{(1+\beta)}{2} n\left(\left(\begin{array}{c}
n \\
k-1
\end{array}\right) p\right)^{2}
$$

Por 3.2 e 3.4 ,

$$
\sum_{\left\{S_{1}, S_{2}\right\} \in B}\left|N\left(S_{1}\right) \cap N\left(S_{2}\right)\right| \leq \frac{\beta C}{2} n\left(\left(\begin{array}{c}
n \\
k-1
\end{array}\right) p\right)^{2}
$$

Substituindo (3.7), 3.8) e (3.9) em (3.6), obtemos

$$
\begin{aligned}
\sum_{u \in V_{G}} d(u)^{2} & \leq(\beta+(1+\beta)+\beta C) n\left(\left(\begin{array}{c}
n \\
k-1
\end{array}\right) p\right)^{2} \\
& \leq(1+\gamma) n\left(\left(\begin{array}{c}
n \\
k-1
\end{array}\right) p\right)^{2}
\end{aligned}
$$

onde a última desigualdade segue de $\beta \leq \gamma /(2+C)$. 
Assim, pelo Lema 3.8, concluímos que, para mais de $(1-\delta) n$ vértices $u \in V_{G}$, temos

$$
\left|d(u)-\left(\begin{array}{c}
n \\
k-1
\end{array}\right) p\right|<\delta\left(\begin{array}{c}
n \\
k-1
\end{array}\right) p
$$

como queríamos.

Para concluir a prova precisamos verificar o item (ii). A estratégia é a mesma que utilizamos na verificação do item (i).

$$
\begin{aligned}
\sum_{\{u, v\} \in\left(\begin{array}{c}
V_{G} \\
2
\end{array}\right)}|N(u) \cap N(v)| & =\sum_{S \in\left(\begin{array}{l}
V_{G} \\
k-1
\end{array}\right)}\left(\begin{array}{c}
|N(S)| \\
2
\end{array}\right) \\
& \geq \sum_{S \in\left(\begin{array}{l}
V_{G} \\
k-1
\end{array}\right):|N(S)| \geq(1-\beta) n p}\left(\begin{array}{c}
|N(S)| \\
2
\end{array}\right) \\
& \geq(1-\beta)\left(\begin{array}{c}
n \\
k-1
\end{array}\right)\left(\begin{array}{c}
(1-\beta) p n \\
2
\end{array}\right) \\
& \geq(1-\beta)^{4}\left(\begin{array}{l}
n \\
2
\end{array}\right)\left(\begin{array}{c}
n \\
k-1
\end{array}\right) p^{2} \\
& \geq(1-\gamma)\left(\begin{array}{c}
n \\
2
\end{array}\right)\left(\begin{array}{c}
n \\
k-1
\end{array}\right) p^{2} .
\end{aligned}
$$

onde a primeira desigualdade é trivial e a segunda segue de (3.3). Na terceira, aplicamos o Lema 3.9 com parâmetros $\varepsilon=\beta, r=2$ e $\alpha=(1-\beta) p$, e a última desigualdade segue de $\beta \leq 1-(1-\gamma)^{1 / 4}$.

Provaremos uma desigualdade que, juntamente com (3.11), nos permite fazer uso do Lema 3.8 .

$$
\begin{aligned}
\sum_{\{u, v\} \in\left(\begin{array}{c}
V_{G} \\
2
\end{array}\right)}|N(u) \cap N(v)|^{2} & =\sum_{S_{1}, S_{2} \in\left(\begin{array}{c}
V_{G} \\
k-1
\end{array}\right)}\left(\begin{array}{c}
\left|N\left(S_{1}\right) \cap N\left(S_{2}\right)\right| \\
2
\end{array}\right) \\
& =2 \sum_{\left\{S_{1}, S_{2}\right\} \in\left(\begin{array}{c}
\left(\begin{array}{c}
V_{G} \\
k \\
2
\end{array}\right) \\
2
\end{array}\right)}\left(\begin{array}{c}
\left|N\left(S_{1}\right) \cap N\left(S_{2}\right)\right| \\
2
\end{array}\right)+\sum_{S_{1} \in\left(\begin{array}{c}
V_{G} \\
k-1
\end{array}\right)}\left(\begin{array}{c}
\left|N\left(S_{1}\right)\right| \\
2
\end{array}\right) .
\end{aligned}
$$

Limitaremos os dois termos da igualdade acima. Por (3.1),

$$
\sum_{S_{1} \in\left(\begin{array}{c}
V_{G} \\
k-1
\end{array}\right)}\left(\begin{array}{c}
\left|N\left(S_{1}\right)\right| \\
2
\end{array}\right) \leq\left(\begin{array}{c}
n \\
k-1
\end{array}\right)\left(\begin{array}{c}
C n p \\
2
\end{array}\right) .
$$

Uma aplicação do Lema 3.10 com parâmetros $\varepsilon=\beta, r=2$ e $b=C p$ implica que

$$
\left(\begin{array}{c}
n \\
k-1
\end{array}\right)\left(\begin{array}{c}
C n p \\
2
\end{array}\right) \leq(1+\beta) C^{2}\left(\begin{array}{l}
n \\
2
\end{array}\right)\left(\begin{array}{c}
n \\
k-1
\end{array}\right) p^{2} .
$$

Como $p \geq\left((1+\beta) C^{2}(k-1)^{k-1} / \beta\right)^{1 / 2} n^{-(k-1) / 2}$, concluímos que $(1+\beta) C^{2} \leq \beta\left(\begin{array}{c}n \\ k-1\end{array}\right) p^{2}$. 
Portanto,

$$
\sum_{S_{1} \in\left(\begin{array}{c}
V_{G} \\
k-1
\end{array}\right)}\left(\begin{array}{c}
\left|N\left(S_{1}\right)\right| \\
2
\end{array}\right) \leq \beta\left(\begin{array}{c}
n \\
2
\end{array}\right)\left(\left(\begin{array}{c}
n \\
k-1
\end{array}\right) p^{2}\right)^{2} .
$$

Para limitar o termo remanescente, defina

$$
A=\left\{\left\{S_{1}, S_{2}\right\} \in\left(\begin{array}{c}
V_{G} \\
k-1 \\
2
\end{array}\right):\left|N\left(S_{1}\right) \cap N\left(S_{2}\right)\right| \leq(1+\beta) n p^{2}\right\}
$$

e também defina

$$
B=\left\{\left\{S_{1}, S_{2}\right\} \in\left(\begin{array}{c}
\left(\begin{array}{c}
V_{G} \\
k-1 \\
2
\end{array}\right) \\
2
\end{array}\right):\left|N\left(S_{1}\right) \cap N\left(S_{2}\right)\right|>(1+\beta) n p^{2}\right\} .
$$

Note que

$$
\begin{aligned}
\sum_{\left\{S_{1}, S_{2}\right\} \in\left(\begin{array}{c}
\left(\begin{array}{c}
V_{G} \\
k-1
\end{array}\right) \\
2
\end{array}\right)}\left(\begin{array}{c}
\left|N\left(S_{1}\right) \cap N\left(S_{2}\right)\right| \\
2
\end{array}\right)= & \sum_{\left\{S_{1}, S_{2}\right\} \in A}\left(\begin{array}{c}
\left|N\left(S_{1}\right) \cap N\left(S_{2}\right)\right| \\
2
\end{array}\right) \\
& +\sum_{\left\{S_{1}, S_{2}\right\} \in B}\left(\begin{array}{c}
\left|N\left(S_{1}\right) \cap N\left(S_{2}\right)\right| \\
2
\end{array}\right) .
\end{aligned}
$$

Aplicando o Lema 3.10 com parâmetros $\varepsilon=\beta, r=2$ e $b=(1+\beta) p^{2}$, obtemos

$$
\begin{aligned}
\sum_{\left\{S_{1}, S_{2}\right\} \in A}\left(\begin{array}{c}
\left|N\left(S_{1}\right) \cap N\left(S_{2}\right)\right| \\
2
\end{array}\right) & \leq \frac{1}{2}\left(\begin{array}{c}
n \\
k-1
\end{array}\right)^{2}\left(\begin{array}{c}
(1+\beta) p^{2} n \\
2
\end{array}\right) \\
& \leq \frac{(1+\beta)^{3}}{2}\left(\begin{array}{l}
n \\
2
\end{array}\right)\left(\left(\begin{array}{c}
n \\
k-1
\end{array}\right) p^{2}\right)^{2} .
\end{aligned}
$$

Por 3.2), 3.4 e pelo Lema 3.10 aplicado com parâmetros $\varepsilon=\beta, r=2$ e $b=C p^{2}$,

$$
\begin{aligned}
\sum_{S_{1}, S_{2} \in B}\left(\begin{array}{c}
\left|N\left(S_{1}\right) \cap N\left(S_{2}\right)\right| \\
2
\end{array}\right) & \leq \frac{\beta}{2}\left(\begin{array}{c}
n \\
k-1
\end{array}\right)^{2}\left(\begin{array}{c}
C p^{2} n \\
2
\end{array}\right) \\
& \leq \frac{\beta(1+\beta) C^{2}}{2}\left(\begin{array}{l}
n \\
2
\end{array}\right)\left(\left(\begin{array}{c}
n \\
k-1
\end{array}\right) p^{2}\right)^{2}
\end{aligned}
$$

Substituindo 3.13), 3.14 e 3.15) em 3.12, obtemos

$$
\begin{aligned}
\sum_{\{u, v\} \in\left(\begin{array}{c}
V_{G} \\
2
\end{array}\right)}|N(u) \cap N(v)|^{2} & \leq\left(\beta+(1+\beta)^{3}+\beta(1+\beta) C^{2}\right)\left(\begin{array}{c}
n \\
2
\end{array}\right)\left(\left(\begin{array}{c}
n \\
k-1
\end{array}\right) p^{2}\right)^{2} \\
& \leq(1+\gamma)\left(\begin{array}{c}
n \\
2
\end{array}\right)\left(\left(\begin{array}{c}
n \\
k-1
\end{array}\right) p^{2}\right)^{2}
\end{aligned}
$$

onde a última desigualdade segue da escolha de $\beta$.

Equações (3.11) e (3.16) podem ser vistas como as condições requeridas pelo Lema 3.8 . 
Assim, concluímos que, para mais de $(1-\delta)\left(\begin{array}{l}n \\ 2\end{array}\right)$ pares de vértices $\{u, v\} \in\left(\begin{array}{c}V_{G} \\ 2\end{array}\right)$, temos

$$
|| N(u) \cap N(v)\left|-\left(\begin{array}{c}
n \\
k-1
\end{array}\right) p^{2}\right|<\delta\left(\begin{array}{c}
n \\
k-1
\end{array}\right) p^{2} .
$$

\subsection{Lema de Extensão e corolários}

Nesta seção, enunciamos e provamos um resultado que chamamos de Lema de Extensão. Desse lema, derivamos dois corolários, essenciais para a prova do Lema 3.7.

\subsubsection{Lema de Extensão}

Primeiramente, definimos alguns conceitos e enunciamos o lema. Feito isso, provamos dois resultados que, juntos, compõem a prova do Lema de Extensão, que é apresentada no final desta subseção.

Considere hipergrafos $k$-uniformes $G$ e $H$. Dadas sequências $F=\left(f_{1}, \ldots, f_{\ell}\right) \in V_{H}^{\ell}$ e $X=\left(x_{1}, \ldots, x_{\ell}\right) \in V_{G}^{\ell}$, defina $\mathcal{I}(H, G, F, X)$ como o conjunto das imersões $f \in \mathcal{I}(H, G)$ tais que $f\left(f_{i}\right)=x_{i}$ para todo $1 \leq i \leq \ell$. Ademais, escrevemos $Y^{\text {con }}=\left\{y_{1}, \ldots, y_{\ell}\right\}$ para o conjunto dos vértices que fazem parte da sequência $Y=\left(y_{1}, \ldots, y_{\ell}\right)$. Dizemos que um subconjunto $V^{\prime} \subset V_{H}$ é estável se não existe aresta de $H$ completamente contida em $V^{\prime}$, i.e., $E\left(H\left[V^{\prime}\right]\right)=\emptyset$.

Seja $H$ um hipergrafo com $m$ vértices. Uma ordenação de $V_{H}$ é simplesmente uma sequência $\left(v_{1}, \ldots, v_{m}\right) \in V_{H}^{m}$. Dizemos que $H$ é $d$-degenerado se existe uma ordenação $\left(v_{1}, \ldots, v_{m}\right)$ de seus vértices de modo que $d_{H_{i}}\left(v_{i}\right) \leq d$ para todo $1 \leq i \leq m$, onde $H_{i}=H\left[\left\{v_{1}, \ldots, v_{i}\right\}\right]$. Nesse caso, dizemos que $\left(v_{1}, \ldots, v_{m}\right)$ é uma ordenação $d$-degenerada dos vértices de $H$. Dada uma sequência $F \in V_{H}^{\ell}$, denotamos por $\omega(H, F)$ a quantidade de arestas de $H$ que não estão contidas em $F^{\text {con }}$, isto é, $\omega(H, F)=\left|E_{H}\right|-\left|E\left(H\left[F^{\text {con }}\right]\right)\right|$.

Lema 3.13 (Lema de Extensão). Sejam $G$ e $H$ hipergrafos $k$-uniformes, onde $H$ é linear, $\left|V_{H}\right|=m,\left|V_{G}\right|=n$ e $p=p(n)=e(G) /\left(\begin{array}{l}n \\ k\end{array}\right)$. Suponha que $0 \leq \ell \leq \max \left\{k, d_{H}\right\}$, e sejam $F \in V_{H}^{\ell}$ e $X \in V_{G}^{\ell}$ fixos. Considere uma constante $C>1$ e suponha que $G \in \operatorname{LMT}^{k}\left(C, D_{H}\right)$. Então

$$
|\mathcal{I}(H, G, F, X)| \leq C^{m-\ell} n^{m-\ell} p^{\omega(H, F)} .
$$

Em particular, se $F^{\mathrm{con}} \subset V_{H}$ é estável, então $|\mathcal{I}(H, G, F, X)| \leq C^{m-\ell} n^{m-\ell} p^{e(H)}$.

As seguintes duas afirmativas compõem a prova do Lema 3.13 . O Lema 3.14 é semelhante ao Lema 3.13 , com a suposição adicional de que existe uma ordenação $D_{H}$-degenerada $\left(v_{1}, \ldots, v_{m}\right)$ de $V_{H}$ tal que $F^{\text {con }}=\left\{v_{1}, \ldots, v_{\ell}\right\}$. Já o Lema 3.15 confirma que tal ordenação existe. 
Lema 3.14. Sejam $G$ e $H$ hipergrafos $k$-uniformes, onde $H$ é linear, com $\left|V_{H}\right|=m,\left|V_{G}\right|=n$ e $p=p(n)=e(G) /\left(\begin{array}{l}n \\ k\end{array}\right)$. Suponha que $0 \leq \ell \leq \max \left\{k, d_{H}\right\}$, e sejam $F \in V_{H}^{\ell}$ e $X \in V_{G}^{\ell}$ fixos. Considere uma constante $C>1$ e suponha que $G \in \operatorname{LMT}^{k}\left(C, D_{H}\right)$. Se existe uma ordenação $D_{H}$-degenerada $v_{1}, \ldots, v_{m}$ de $V_{H}$ tal que $F^{\text {con }}=\left\{v_{1}, \ldots, v_{\ell}\right\}$, então

$$
|\mathcal{I}(H, G, F, X)| \leq C^{m-\ell} n^{m-\ell} p^{\omega(H, F)} .
$$

Visão geral da demonstração. Utilizamos indução em $h$, com $\ell \leq h \leq m$, para provar que $\left|\mathcal{I}\left(H_{h}, G, F, X\right)\right| \leq C^{m-\ell} n^{m-\ell} p^{\omega(H, F)}$, onde $H_{h}=H\left[\left\{v_{1}, \ldots, v_{h}\right\}\right]$, de acordo com uma ordenação $D_{H^{-}}$degenerada $\left\{v_{1}, \ldots, v_{m}\right\}$. Assim, como $d_{H_{h}}\left(v_{h}\right) \leq D_{H}$, podemos usar o fato de $G \in \operatorname{LMT}^{k}\left(C, D_{H}\right)$ para limitar a quantidade de imersões $\mathcal{I}\left(H_{h}, G, F, X\right)$ obtidas pela extensão de imersões em $\mathcal{I}\left(H_{h-1}, G, F, X\right)$, que sabemos não ser um conjunto muito grande, por conta da hipótese indutiva.

Demonstração. Considere hipergrafos $k$-uniformes $G$ e $H$, onde $\left|V_{H}\right|=m$ e $H$ é linear, $\left|V_{G}\right|=$ $n$ e $p=p(n)=e(G) /\left(\begin{array}{l}n \\ k\end{array}\right)$. Fixe $0 \leq \ell \leq \max \left\{k, d_{H}\right\}, F \in V_{H}^{\ell}$ e $X \in V_{G}^{\ell}$. Considere uma constante fixa $C>1$ e suponha que $G \in \operatorname{LMT}^{k}\left(C, D_{H}\right)$.

Suponha que existe uma ordenação $D_{H}$-degenerada $L=\left(v_{1}, \ldots, v_{m}\right)$ de $V_{H}$ tal que $F^{\text {con }}=\left\{v_{1}, \ldots, v_{\ell}\right\}$. Vamos provar por indução em $h$ que, para todo $\ell \leq h \leq m$, temos

$$
\left|\mathcal{I}\left(H_{h}, G, F, X\right)\right| \leq C^{h-\ell} n^{h-\ell} p^{\omega\left(H_{h}, F\right)}
$$

onde $H_{h}=H\left[\left\{v_{1}, \ldots, v_{h}\right\}\right]$. Se $h=\ell$, o resultado é trivial. Suponha que $\ell<h \leq m$ e que (3.17) é verdade para valores menores de $h$. Note que $d_{H_{h}}\left(v_{h}\right) \leq D_{H}$, dado que $L$ é $D_{H}$-degenerada. Mas sabendo que $G \in \operatorname{LMT}^{k}\left(C, D_{H}\right)$, qualquer imersão de a $H_{h-1}$ pode ser estendida para uma imersão de $H_{h}$ de, no máximo, $C n p^{d_{H_{h}}\left(v_{h}\right)}$ maneiras diferentes. Como $\omega\left(H_{h}, F\right)=\omega\left(H_{h-1}, F\right)+d_{H_{h}}\left(v_{h}\right)$, aplicando a hipótese indutiva, concluímos que

$$
\begin{aligned}
\left|\mathcal{I}\left(H_{h}, G, F, X\right)\right| & \leq C n p^{d_{H_{h}}\left(v_{h}\right)}\left|\mathcal{I}\left(H_{h-1}, G, F, X\right)\right| \\
& \leq C n p^{d_{H_{h}}\left(v_{h}\right)} C^{h-1-\ell} n^{h-1-\ell} p^{\omega\left(H_{h-1}, F\right)} \\
& =C^{h-\ell} n^{h-\ell} p^{\omega\left(H_{h}, F\right)} .
\end{aligned}
$$

Tomando $h=m$, a prova está terminada.

Seja $H$ um hipergrafo linear $k$-uniforme com $m$ vértices e considere uma ordenação $L=\left(v_{1}, \ldots, v_{m}\right)$ dos vértices de $H$. Dado um conjunto $W \subset V_{H}$, escrevemos $L \backslash W$ para a ordenação de $V_{H} \backslash W$ obtida de $L$ após a remoção dos vértices de $W$. Por exemplo, se $W=\left\{v_{1}, v_{3}, v_{m}\right\}$, então $L \backslash W=\left(v_{2}, v_{4}, v_{5}, \ldots, v_{m-1}\right)$. Dada uma sequência de vértices $Y=\left(v_{i_{1}}, \ldots, v_{i_{\ell}}\right) \in V_{H}^{\ell}$, escrevemos a sequência $L^{\prime}=\left(v_{i_{1}} v_{i_{2}} \ldots . v_{i_{\ell}} \cdot\left\{L \backslash Y^{\text {con }}\right\}\right)$ para denotar a ordenação $L^{\prime}$ de $V_{H}$ obtida quando removemos $Y$ e a colocamos antes dos elementos de $L$. 
Lema 3.15. Seja $H$ um hipergrafo linear $k$-uniforme tal que existe uma ordenação $d_{H^{-}}$ degenerada dos vértices de $H$. Suponha que $0 \leq \ell \leq \max \left\{k, d_{H}\right\}$. Se $F \in V_{H}^{\ell}$, então existe uma ordenação $D_{H}$-degenerada $\left(v_{1}, \ldots, v_{\left|V_{H}\right|}\right) \operatorname{com} F^{\text {con }}=\left\{v_{1}, \ldots, v_{\ell}\right\}$.

Demonstração. Por simplicidade, defina $d=d_{H}$. Se $D_{H}=\Delta(H)$, então qualquer ordenação dos vértices de $H$ é $D_{H}$-degenerada. Assim, suponha que $D_{H}=k d$. Note que o resultado é trivial se $F=\emptyset$. Desta forma, assumimos que $1 \leq|F|=\ell \leq \max \{k, d\}$.

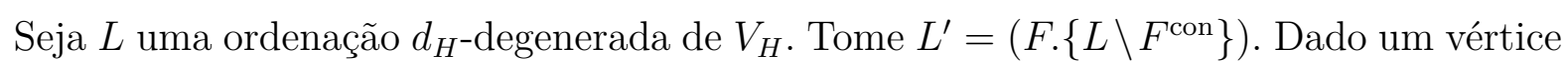
$x$ de $H$, definimos o grau à esquerda de $x$ em $L^{\prime}$ como a quantidade de arestas tal que $x$ é o elemento mais à direita da aresta, levando em conta a ordenação $L^{\prime}$. Deste modo, note que o grau à esquerda de cada vértice de $H$ em $L^{\prime}$ é no máximo $|F|+d$, pois $L$ é uma ordenação $d$-degenerada e, pela linearidade de $H$, tal vértice pode estar presente em no máximo $|F|$ arestas que contêm vértices de $F$.

Suponha que $d>k$. Assim, $|F| \leq d$ e o grau à esquerda de qualquer vértice de $H$ em $L^{\prime}$

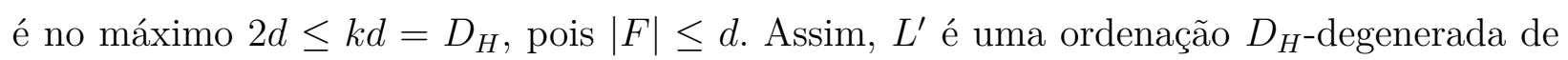
$V_{H}$.

Suponha que $2 \leq d \leq k$. Logo, $|F| \leq k$. Portanto, o grau à esquerda de cada vértice de $H$

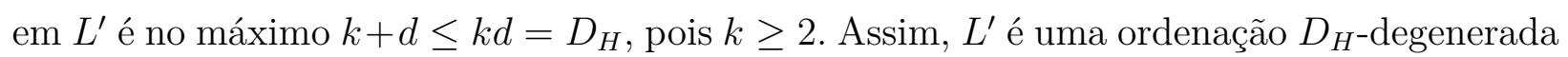
de $V_{H}$.

Por fim, seja $d=1$. Aqui, temos $|F| \leq k$. Note que a única possibilidade de algum vértice $x$ ter grau à esquerda maior que $k d=k$ em $L^{\prime}$ é se $x$ pertencer a uma aresta que contém um vértice $f$, para cada vértice $f \in F$, onde $|F|=k$ e $f$ é o elemento que está mais à direita na aresta. Mas assim, só pode existir um vértice $x$ com essa característica, dado que $d=1$. Então, considere a ordenação $L^{\prime \prime}=\left(F . x .\left\{L \backslash\left\{F^{\text {con }} \cup\{x\}\right\}\right)\right.$. Assim, é claro que todo vértice de $H$ possui grau à esquerda no máximo $2=k d=D_{H}$.

Prova do Lema de Extensão (Lema 3.13). Sejam $G$ e $H$ hipergrafos $k$-uniformes onde $H$ é linear, com $|V(H)|=m,|V(G)|=n$ e $p=p(n)=e(G) /\left(\begin{array}{l}n \\ k\end{array}\right)$. Suponha $0 \leq \ell \leq \max \left\{k, d_{H}\right\}$, e tome $F \in V(H)^{\ell}$ e $X \in V(G)^{\ell}$ fixos. Seja $C>1$ uma constante e suponha que $G \in$ $\operatorname{LMT}^{k}\left(C, D_{H}\right)$.

É fácil ver que existe uma ordenação $d_{H^{-}}$degenerada $L$ dos vértices de $H$. Assim, pelo

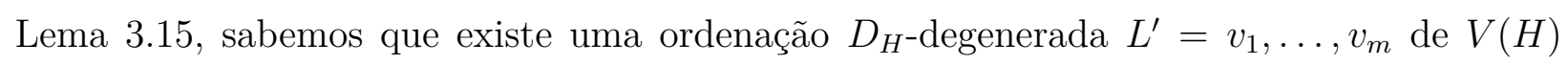
com $F^{\text {con }}=\left\{v_{1}, \ldots, v_{\ell}\right\}$. Então, podemos aplicar Lema 3.14 para concluir que

$$
|\mathcal{I}(H, G, F, X)| \leq C^{m-\ell} n^{m-\ell} p^{\omega(H, F)} .
$$

\subsubsection{Corolários do Lema de Extensão}

Dados hipergrafos $k$-uniformes $G$ e $H$, escrevemos $\mathcal{I}^{\text {ni }}(H, G)$ para o conjunto de imersões não induzidas de $\mathcal{I}(H, G)$. Ademais, denotamos por $\mathcal{I}^{\text {ind }}(H, G)$ o conjunto de imersões induzidas 
de $\mathcal{I}(H, G)$. O seguinte corolário limita superiormente a cardinalidade de $\mathcal{I}^{\text {ni }}(H, G)$, quando $H$ é linear e $G$ possui algumas propriedades.

Corolário 3.16. Para todos $C \geq 1, m \geq 1, k \geq 2, \eta>0$ e $p=p(n)=o(1)$, existe $n_{0}>0$ tal que, para todos os hipergrafos $k$-uniformes $G$ e $H$, onde $\left|V_{G}\right|=n$ e $H$ é linear com $\left|V_{H}\right|=m$, se $G \in \operatorname{LMT}^{k}\left(C, D_{H}\right),|E(G)| \leq n^{k} p$ e $n \geq n_{0}$, então

$$
\left|\mathcal{I}^{\text {ni }}(H, G)\right|<\eta n^{m} p^{e(H)}
$$

Visão geral da demonstração do Corolário 3.16. Sabemos que qualquer imersão não induzida mapeia pelo menos uma não-aresta de $H$ em uma aresta de $G$. Assim, aplicamos o Lema 3.13 . pondo $F$ como sendo uma ordenação de uma não-aresta de $H$, e pondo $X$ como sendo uma ordenação de uma aresta de $G$. Para finalizar a prova, contamos de quantas maneiras podemos escolher tal não-aresta de $H$ e tal aresta de $G$.

Demonstração. Fixe $C \geq 1, m \geq 1, k \geq 2, \eta>0$ e tome $p=p(n)=o(1)$. Sejam hipergrafos $k$-uniformes $G$ e $H$, onde $\left|V_{G}\right|=n$ e $H$ é linear com $\left|V_{H}\right|=m$ e seja $n_{0}$ um inteiro positivo tal que se $n \geq n_{0}$ então $p(n)<\eta\left((k !)^{2} C^{m-k}\left(\begin{array}{c}m \\ k\end{array}\right)\right)^{-1}$. Suponha que $n \geq n_{0}$ e $G \in \operatorname{LMT}^{k}\left(C, D_{H}\right)$ $\operatorname{com}|E(G)| \leq n^{k} p$.

Se $k>m$, o resultado segue trivialmente, dado que neste caso o conjunto de arestas de $H$ é vazio. Portanto, assumimos $k \leq m$. Fixe uma aresta $\left\{x_{1}, \ldots, x_{k}\right\} \in E(G)$ e uma não-aresta $\left\{v_{1}, \ldots, v_{k}\right\} \in\left(\begin{array}{c}V_{H} \\ k\end{array}\right)$ de $H$. Aplicamos o Lema 3.13 pondo $F=\left(v_{1}, \ldots, v_{k}\right)$ e $G=\left(x_{1}, \ldots, x_{k}\right)$, obtendo que o número de imersões $f$ de $V_{H}$ em $V_{G}$ tais que $f\left(v_{i}\right)=x_{i}$ para $1 \leq i \leq k$ é limitado por cima por $C^{m-k} n^{m-k} p^{E_{H}}$. Mas note que existem no máximo $n^{k} p$ escolhas para $\left\{x_{1}, \ldots, x_{k}\right\}$ em $E(G)$ e no máximo $\left(\begin{array}{c}m \\ k\end{array}\right)$ escolhas para $\left\{v_{1}, \ldots, v_{k}\right\}$ em $\left(\begin{array}{c}V_{H} \\ k\end{array}\right)$. Assim, podemos escolher $\left(x_{1}, \ldots, x_{k}\right)$ e $\left(v_{1}, \ldots, v_{k}\right)$, respectivamente, de $k ! n^{k} p$ e $k !\left(\begin{array}{c}m \\ k\end{array}\right)$ maneiras. Portanto,

$$
\begin{aligned}
\left|\mathcal{I}^{\mathrm{ni}}(H, G)\right| & \leq\left(k ! n^{k} p\right)\left(k !\left(\begin{array}{c}
m \\
k
\end{array}\right)\right) C^{m-k} n^{m-k} p^{e(H)} \\
& =\left((k !)^{2} C^{m-k}\left(\begin{array}{c}
m \\
k
\end{array}\right) p\right) n^{m} p^{e(H)} \\
& <\eta n^{m} p^{e(H)}
\end{aligned}
$$

onde a última desigualdade segue da escolha de $n_{0}$.

Sejam $G$ e $H$ hipergrafos $k$-uniformes e considere $X \subset\left(\begin{array}{c}V_{H} \\ k-1\end{array}\right)$. Se $f$ é uma imersão de $H$ em $G$, denotamos por $f_{k-1}(X)$ a família de conjuntos $\left\{f\left(x_{1}\right), \ldots, f\left(x_{k-1}\right)\right\}$, para todo $\left\{x_{1}, \ldots, x_{k-1}\right\} \in X$.

Para $1 \leq r \leq k$, dado $X=\left\{X_{1}, \ldots, X_{r}\right\}$, onde $X_{i}=\left\{x_{i, 1}, \ldots, x_{i, k-1}\right\} \in\left(\begin{array}{c}V_{H} \\ k-1\end{array}\right)$, para $1 \leq i \leq r$, definimos $X^{\text {con }}=\left\{x_{1,1}, \ldots, x_{1, k-1}, \ldots, x_{r, 1}, \ldots, x_{r, k-1}\right\}$. 
Seja $G_{n}$ um hipergrafo $k$-uniforme com $n$ vértices. Para $r \geq 1$ e $\delta>0$, defina

$$
\mathcal{B}(\delta, r)=\left\{X \in\left(\begin{array}{c}
V_{G} \\
k-1
\end{array}\right):|| N_{G_{n}}(X)\left|-n p^{r}\right| \geq \delta n p^{r}\right\},
$$

Em outras palavras, $\mathcal{B}(\delta, r)$ é composto por famílias $X$ com $r$ membros de $\left(\begin{array}{c}V_{G} \\ k-1\end{array}\right)$ tais que a quantidade de vizinhos comuns dos membros de $X$ não é próxima de $n p^{r}$. Dizemos que $X \in\left(\begin{array}{c}\left(\begin{array}{c}V_{G} \\ k\end{array}\right) \\ r\end{array}\right)$ é $\delta$-ruim se $X \in \mathcal{B}_{\text {est }}(\delta, r)$, onde $\mathcal{B}_{\text {est }}(\delta, r)=\left\{X \in \mathcal{B}(\delta, r): X^{\text {con }}\right.$ é estável em $\left.G_{n}\right\}$.

Seja $H$ um hipergrafo com $m$ vértices e seja $\left(v_{1}, \ldots, v_{m}\right)$ uma ordenação $d_{H}$-degenerada de $V_{H}$. Defina $H_{i}=H\left[v_{1}, \ldots, v_{i}\right]$. Dizemos que uma imersão $f: V\left(H_{h-1}\right) \rightarrow V\left(G_{n}\right)$ é $\delta$-limpa se o conjunto $f_{k-1}\left(N_{H_{h}}\left(v_{h}\right)\right)$ não é $\delta$-ruim. Isto é, $f_{k-1}\left(N_{H_{h}}\left(v_{h}\right)\right) \notin \mathcal{B}_{\text {est }}\left(\delta, d_{H_{h}}\left(v_{h}\right)\right)$. Se $f: V\left(H_{h-1}\right) \rightarrow V\left(G_{n}\right)$ não é $\delta$-limpa, dizemos que $f$ é $\delta$-poluída. Denotamos o conjunto das imersões $f \in \mathcal{I}\left(H_{h-1}, G_{n}\right)$ tais que $f$ é $\delta$-poluída por $\mathcal{I}_{\delta \text {-pol }}\left(H_{h-1}, G_{n}\right)$. Similarmente, denotamos por $\mathcal{I}_{\delta \text {-limpa }}\left(H_{h-1}, G_{n}\right)$ o conjunto das imersões $f \in \mathcal{I}\left(H_{h-1}, G_{n}\right)$ tais que $f$ é $\delta$-limpa.

Corolário 3.17. Sejam $\delta>0, C>1$ e $m \geq 4$ constantes fixas. Considere um hipergrafo linear $k$-uniforme $H$ livre de conectores com $m$ vértices e seja $\left(v_{1}, \ldots, v_{m}\right)$ uma ordenação $d_{H}$-degenerada de $V_{H}$. Suponha que $1<h \leq m$ e defina $r=d_{H_{h}}\left(v_{h}\right)$. Se $G_{n}$ é $\left(C, D_{H}, d_{H}, \delta\right)$ pseudoaleatório, então

$$
\left|\mathcal{I}_{\delta-\mathrm{pol}}\left(H_{h-1}, G_{n}\right)\right| \leq \delta\left(r !((k-1) !)^{r} C^{h-1-r(k-1)}\right) n^{h-1} p^{e\left(H_{h-1}\right)},
$$

onde $p=e\left(G_{n}\right) /\left(\begin{array}{l}n \\ k\end{array}\right)$.

Visão geral da demonstração do Corolário 3.17. Se uma imersão $f \in \mathcal{I}\left(H_{h-1}, G_{n}\right)$ é $\delta$ poluída, então $f_{k-1}\left(N_{H_{h}}\left(v_{h}\right)\right)$ é $\delta$-ruim. Mas podemos limitar a quantidade de conjuntos $\delta$-ruins utilizando a propriedade $\mathrm{CG}_{\delta}^{k}\left(d_{H}\right)$. Desta forma, finalizamos a prova utilizando o Lema 3.13, não esquecendo que $H$ é linear e livre de conectores, para estimar de quantas maneiras podemos realizar a imersão da vizinhança de $v_{H}$ em conjuntos $\delta$-ruins de $G$.

Demonstração. Fixe constantes $\delta>0, C>1$ e $m \geq 4$. Seja $H$ um hipergrafo linear $k$-uniforme livre de conectores com $m$ vértices e considere uma ordenação $d_{H^{-}}$degenerada $\left(v_{1}, \ldots, v_{m}\right)$ de $V_{H}$. Considere $1<h \leq m$ e defina $r=d_{H_{h}}\left(v_{h}\right)$. Suponha que $G_{n} \in \mathrm{CG}_{\delta}^{k}\left(d_{H}\right)$ e $G_{n} \in \operatorname{LMT}^{k}\left(C, D_{H}\right)$.

Sabemos que uma imersão $f: V\left(H_{h-1}\right) \rightarrow V\left(G_{n}\right)$ é $\delta$-poluída se $f_{k-1}\left(N_{H_{h}}\left(v_{h}\right)\right) \in \mathcal{B}_{\text {est }}(\delta, r)$. Fixe uma sequência $F=\left(T_{1}, \ldots, T_{r}\right)$ composta de $r$ conjuntos de $k-1$ vértices de $H$ tal que $\left\{T_{1}, \ldots, T_{r}\right\}=N_{H_{h}}\left(v_{h}\right)$ e seja $F_{\text {ord }}=\left(u_{1,1}, \ldots, u_{1, k-1}, u_{2,1}, \ldots, u_{2, k-1}, \ldots, u_{r, 1} \ldots, u_{r, k-1}\right)$ uma ordenação de tais vértices tal que $T_{i}=\left\{u_{i, 1}, \ldots, u_{i, k-1}\right\}$ para todo $1 \leq i \leq r$. Portanto,

$$
\mathcal{I}_{\delta-\text { pol }}\left(H_{h-1}, G_{n}\right)=\bigcup_{X}\left(\bigcup_{X_{\text {ord }}} \mathcal{I}\left(H_{h-1}, G_{n}, F_{\text {ord }}, X_{\text {ord }}\right)\right)
$$


onde a primeira união é sobre todas as famílias $X=\left\{S_{1}, \ldots, S_{r}\right\} \in \mathcal{B}_{\text {est }}(\delta, r)$ de $r$ conjuntos de $k-1$ vértices e a segunda união é sobre todas as possíveis ordenações de $S_{i}$, para $1 \leq i \leq r$, e toda ordenação de $X$. Portanto, podemos limitar a quantidade de imersões $\left|\mathcal{I}_{\delta-\text { pol }}\left(H_{h-1}, G_{n}\right)\right|$ como segue.

$$
\left|\mathcal{I}_{\delta-\text { pol }}\left(H_{h-1}, G_{n}\right)\right| \leq \sum_{X} \sum_{X_{\text {ord }}}\left|\mathcal{I}\left(H_{h-1}, G_{n}, F_{\text {ord }}, X_{\text {ord }}\right)\right|
$$

Dadas $F_{\text {ord }}$ e $X_{\text {ord }}$, como $N_{H_{h}}^{\text {con }}\left(v_{h}\right)$ é estável em $H_{h}$ (isso é verdade, uma vez que $H_{h} \subset H$ é livre de conectores e linear) e $G_{n} \in \operatorname{LMT}^{k}\left(C, D_{H}\right)$, sabemos, pelo Lema 3.13, que

$$
\left|\mathcal{I}\left(H_{h-1}, G_{n}, F_{\text {ord }}, X_{\text {ord }}\right)\right| \leq C^{h-1-r(k-1)} n^{h-1-r(k-1)} p^{e\left(H_{h-1}\right)},
$$

pois $\left|F_{\text {ord }}\right|=r(k-1)$. Como $r=d_{H_{h}}\left(v_{h}\right) \leq d_{H}$, podemos concluir da propriedade $\mathrm{CG}_{\delta}^{k}\left(d_{H}\right)$ que $\left|\mathcal{B}_{\text {est }}(\delta, r)\right| \leq \delta n^{r(k-1)}$. Assim, a primeira soma possui no máximo $\delta n^{r(k-1)}$ termos. A segunda soma é sobre não mais que $r !((k-1) !)^{r}$ termos. Portanto,

$$
\left|\mathcal{I}_{\delta-\mathrm{pol}}\left(H_{h-1}, G_{n}\right)\right| \leq \delta\left(r !((k-1) !)^{r} C^{h-1-r(k-1)}\right) n^{h-1} p^{e\left(H_{h-1}\right)} .
$$

\subsection{Provas dos lemas principais}

Provamos nesta seção os Lemas 3.6 e 3.7, que são os resultados que compõem a prova do Teorema 3.5 (veja Seção 3.1).

\subsubsection{Prova do Lema $\mathrm{CG}_{2 \rightarrow t}$}

Visão geral da demonstração do Lema $\mathrm{CG}_{2 \rightarrow t}$ (Lema 3.6). Tome $t \geq 2$ e fixe $2 \leq r \leq t$. Queremos aplicar o Lema 3.8 com parâmetros $a=n p^{r}$ e $N=\left(\begin{array}{c}n \\ k-1 \\ r\end{array}\right)$, onde colocamos $a_{i}=\left|N\left(S_{1} \cap \ldots \cap S_{r}\right)\right|$ para cada $i=\left\{S_{1}, \ldots, S_{r}\right\} \in\left(\begin{array}{c}\left(\begin{array}{c}G_{n} \\ k-1 \\ r\end{array}\right) \\ r\end{array}\right)$. Assim, verificando que as duas condições do Lema 3.8 são válidas, o resultado está provado, dado que a conclusão do lema é precisamente o que queremos provar.

Para verificar a condição $\sum_{i=1}^{N} a_{i} \geq(1-\gamma) N a$, notamos que $\sum_{i=1}^{N} a_{i}=\sum_{u \in V_{G}}\left(\begin{array}{c}d(u) \\ r\end{array}\right)$ e fazemos uso dos Lemas 3.9 e 3.12. Para provar que $\sum_{i=1}^{N} a_{i}{ }^{2} \leq(1+\gamma) N a^{2}$, primeiramente observamos que podemos escrever $\sum_{i=1}^{N} a_{i}{ }^{2} \operatorname{como} \sum_{u, v \in V_{G}}\left(\begin{array}{c}|N(u) \cap N(v)| \\ r\end{array}\right)$. Feito isso, utilizando os Lemas 3.10, 3.11 e 3.12, conseguimos verificar que a condição é válida.

Prova do Lema 3.6. Fixe $t \geq 2, C>1$ e suponha que $p \gg n^{-1 / t}$. Agora, fixe $2 \leq r \leq t$ e $\delta>0$. Considere $\gamma>0$ obtido por uma aplicação do Lema 3.8 com parâmetro $\delta>0$. Faça

$$
\alpha=\min \left\{1-(1-\gamma)^{1 /(r+2)},(1+\gamma / 2)^{1 /(r+1)}-1, \gamma /\left(6 C^{r}(k-1)^{r(k-1)}\right)\right\}
$$


Considere agora $\beta$ e $n_{1}$ obtidos por uma aplicação do Lema 3.12 com parâmetro $\alpha$. Pela suposição sobre $p$, sabemos que existe $n_{2}>0$ tal que $p \geq\left(\alpha^{-1} 2 C^{r}(k-1)^{r(k-1)}\right)^{-1 / r} n^{-1 / r}$ para todo $n \geq n_{2}$. Aplicando o Lema 3.9 com parâmetros $\alpha, r$ e $(1-\alpha) p$, obtemos $n_{3}>0$. Por fim, uma aplicação do Lema $3.10 \operatorname{com} \alpha, r$ e $C(k-1)^{k-1} p$ fornece uma constante $n_{4}>0$.

Assuma $n \geq n_{0}=\min \left\{n_{1}, n_{2}, n_{3}, n_{4}\right\}$. Considere um hipergrafo $k$-uniforme $G=G_{n}$ com $n$ vértices e $\left|E\left(G_{n}\right)\right|=\left(\begin{array}{l}n \\ k\end{array}\right) p$. Suponha que $G_{n} \in \operatorname{LMT}^{k}(C, 2)$ e $G_{n} \in \mathrm{CG}_{\beta}^{k}(2)$. Pelo Lema 3.12 temos o seguinte: para mais de $(1-\alpha) n$ vértices $u \in V_{G}$, segue que

$$
\left|d(u)-\left(\begin{array}{c}
n \\
k-1
\end{array}\right) p\right|<\alpha\left(\begin{array}{c}
n \\
k-1
\end{array}\right) p .
$$

Para mais de $(1-\alpha)\left(\begin{array}{l}n \\ 2\end{array}\right)$ pares de vértices $\{u, v\} \in\left(\begin{array}{c}V_{G} \\ 2\end{array}\right)$, segue que

$$
|| N(u) \cap N(v)\left|-\left(\begin{array}{c}
n \\
k-1
\end{array}\right) p^{2}\right|<\alpha\left(\begin{array}{c}
n \\
k-1
\end{array}\right) p^{2} .
$$

Precisamos verificar que as condições do Lema 3.8 são válidas. Para provar que a primeira das condições é válida, note que

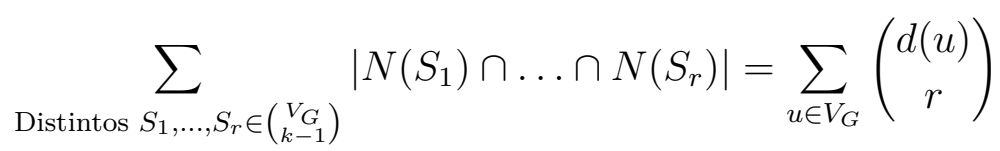

$$
\begin{aligned}
& \geq \sum_{u:|N(u)| \geq(1-\alpha)\left(\begin{array}{c}
n \\
k-1
\end{array}\right) p}\left(\begin{array}{c}
d(u) \\
r
\end{array}\right) \\
& \geq(1-\alpha) n\left(\begin{array}{c}
(1-\alpha) p\left(\begin{array}{c}
n \\
k-1
\end{array}\right) \\
r
\end{array}\right) \\
& \left.\geq(1-\alpha)^{r+2}\left(\begin{array}{c}
n \\
k-1 \\
r
\end{array}\right)\right)\left(n p^{r}\right) \\
& \geq(1-\gamma)\left(\begin{array}{c}
n \\
k-1 \\
r
\end{array}\right)\left(n p^{r}\right),
\end{aligned}
$$

onde a primeira desigualdade é trivial e a segunda segue de (3.18). A terceira segue da aplicação que fizemos do Lema 3.9, e a última segue da escolha de $\alpha$.

Resta mostrar que a condição (ii) do Lema 3.8 é válida. Note que

$$
\begin{aligned}
\sum_{\operatorname{Distintos}}\left|\bigcap_{S_{1}, \ldots, S_{r} \in\left(\begin{array}{c}
V_{G} \\
k-1
\end{array}\right)}^{r} N\left(S_{i}\right)\right|^{2} & =\sum_{u, v \in V_{G}}\left(\begin{array}{c}
|N(u) \cap N(v)| \\
r
\end{array}\right) \\
& =2 \sum_{\{u, v\} \in\left(\begin{array}{c}
V_{G} \\
2
\end{array}\right)}\left(\begin{array}{c}
|N(u) \cap N(v)| \\
r
\end{array}\right)+\sum_{u \in V_{G}}\left(\begin{array}{c}
d(u) \\
r
\end{array}\right) .
\end{aligned}
$$

Vamos estimar separadamente os dois termos do lado direito na igualdade acima. Pelo 
Lema 3.11 .

$$
\sum_{u \in V_{G}}\left(\begin{array}{c}
d(u) \\
r
\end{array}\right) \leq n\left(\begin{array}{c}
C n^{k-1} p \\
r
\end{array}\right) .
$$

Nossa aplicação do Lema 3.10 garante que

$$
n\left(\begin{array}{c}
C n^{k-1} p \\
r
\end{array}\right) \leq n\left(\begin{array}{c}
C(k-1)^{k-1} p\left(\begin{array}{c}
n \\
k-1
\end{array}\right) \\
r
\end{array}\right) \leq C^{\prime}\left(\begin{array}{c}
n \\
k-1 \\
r
\end{array}\right) n p^{r}
$$

onde fazemos $C^{\prime}=(1+\alpha) C^{r}(k-1)^{r(k-1)}$. Como $p \geq\left(C^{\prime} / \alpha\right)^{1 / r} n^{-1 / r}$, podemos concluir que $C^{\prime} \leq \alpha n p^{r}$. Portanto,

$$
\left.\sum_{u \in V_{G}}\left(\begin{array}{c}
d(u) \\
r
\end{array}\right) \leq \alpha\left(\begin{array}{c}
n \\
k-1 \\
r
\end{array}\right)\right)\left(n p^{r}\right)^{2} .
$$

Agora vamos estimar o termo remanescente. Defina

$$
A=\left\{\{u, v\} \in\left(\begin{array}{c}
V_{G} \\
2
\end{array}\right):|N(u) \cap N(v)| \leq(1+\alpha)\left(\begin{array}{c}
n \\
k-1
\end{array}\right) p^{2}\right\} .
$$

Defina também

$$
B=\left\{\{u, v\} \in\left(\begin{array}{c}
V_{G} \\
2
\end{array}\right):|N(u) \cap N(v)|>(1+\alpha)\left(\begin{array}{c}
n \\
k-1
\end{array}\right) p^{2}\right\} .
$$

Note que

$$
\sum_{\{u, v\} \in\left(\begin{array}{c}
V_{G} \\
2
\end{array}\right)}\left(\begin{array}{c}
|N(u) \cap N(v)| \\
r
\end{array}\right)=\sum_{\{u, v\} \in A}\left(\begin{array}{c}
|N(u) \cap N(v)| \\
r
\end{array}\right)+\sum_{\{u, v\} \in B}\left(\begin{array}{c}
|N(u) \cap N(v)| \\
r
\end{array}\right) .
$$

Lema 3.10 aplicado com parâmetros $\alpha, r$ e $(1+\alpha) p^{2}$ implica

$$
\begin{aligned}
\sum_{\{u, v\} \in A}\left(\begin{array}{c}
|N(u) \cap N(v)| \\
r
\end{array}\right) & \leq \frac{n^{2}}{2}\left(\begin{array}{c}
(1+\alpha) p^{2}\left(\begin{array}{c}
n \\
k-1
\end{array}\right) \\
r
\end{array}\right) \\
& \leq \frac{(1+\alpha)^{r+1}}{2}\left(\begin{array}{c}
n \\
k-1 \\
r
\end{array}\right)\left(n p^{r}\right)^{2}
\end{aligned}
$$

Por 3.19), Lema 3.10 aplicado com $\alpha, r$ e $C(k-1)^{k-1} p^{2}$ e Lema 3.11, temos

$$
\begin{aligned}
& \sum_{\{u, v\} \in B}\left(\begin{array}{c}
|N(u) \cap N(v)| \\
r
\end{array}\right) \leq \frac{\alpha}{2} n^{2}\left(\begin{array}{c}
C(k-1)^{k-1} p^{2}\left(\begin{array}{c}
n \\
k-1
\end{array}\right) \\
r
\end{array}\right) \\
& \left.\leq \frac{\alpha(1+\alpha) C^{r}(k-1)^{r(k-1)}}{2}\left(\begin{array}{c}
n \\
k-1 \\
r
\end{array}\right)\right)\left(n p^{r}\right)^{2} .
\end{aligned}
$$

Substituindo (3.24) e 3.25 em (3.23), obtemos um limitante que, ao ser substituído junta- 
mente com (3.22) em (3.21), implica o seguinte.

$$
\begin{aligned}
& \left.\sum_{\text {Distintos }}\left|\bigcap_{S_{1}, \ldots, S_{r} \in\left(\begin{array}{c}
V_{G} \\
k-1
\end{array}\right)} \bigcap_{i=1}^{r} N\left(S_{i}\right)\right|^{2} \leq\left(\alpha+(1+\alpha)^{r+1}+\alpha(1+\alpha) C^{r}(k-1)^{r(k-1)}\right)\left(\begin{array}{c}
n \\
k-1 \\
r
\end{array}\right)\right)\left(n p^{r}\right)^{2} \\
& \leq(1+\gamma)\left(\begin{array}{c}
n \\
k-1 \\
r
\end{array}\right)\left(n p^{r}\right)^{2}
\end{aligned}
$$

onde a última desigualdade segue da escolha de $\alpha$.

Equações 3.20 e 3.26 podem ser vistas como as condições (i) e (ii) do Lema 3.8. Assim, concluímos que, para mais de $(1-\delta)\left(\begin{array}{c}\left(\begin{array}{c}n \\ k-1\end{array}\right) \\ r\end{array}\right)$ conjuntos distintos $S_{1}, \ldots, S_{r} \in\left(\begin{array}{c}V_{G} \\ k-1\end{array}\right)$, temos

$$
|| N\left(S_{1}\right) \cap \ldots \cap N\left(S_{r}\right)\left|-n p^{r}\right|<\delta n p^{r}
$$

\subsubsection{Prova do Lema de contagem modificado}

Visão geral da demonstração do Lema de contagem modificado (Lema 3.7). Utilizamos indução em $h$, com $1 \leq h \leq m$ para provar que || $\mathcal{I}\left(H_{h}, G\right)\left|-n^{h} p^{e\left(H_{h}\right)}\right| \leq \varepsilon n^{h} p^{e\left(H_{h}\right)}$, onde $H_{h}=H\left[\left\{v_{1}, \ldots, v_{h}\right\}\right]$ de acordo com uma ordenação $d_{H}$-degenerada $\left\{v_{1}, \ldots, v_{m}\right\}$. Fazemos uso dos Corolários 3.16 e 3.17 para garantir que a maioria das imersões de $H_{h-1}$ em $G$ são limpas e induzidas e, desta forma, podemos focar somente nesse tipo de imersões.

Considere uma imersão limpa e induzida $f^{\prime}$ de $H_{h-1}$ em $G_{n}$. Como $H$ é linear e livre de conectores, $N_{H_{h}}^{\text {con }}\left(v_{h}\right)$ é estável em $H_{h}$. Mas como $f^{\prime}$ é induzida, sabemos que $f^{\prime}\left(N_{H_{h}}^{\text {con }}\left(v_{h}\right)\right)$ é estável em $G_{n}$. Porém, como $f^{\prime}$ é limpa, podemos concluir que $\mid N_{G_{n}}\left(f_{k-1}^{\prime}\left(N_{H_{h}}\left(v_{h}\right)\right)\right)$ $n p^{r} \mid<\delta n p^{r}$. Desta forma, provamos o limite inferior utilizando esses fatos e fazendo algumas contagens. Para o limite superior, precisamos utilizar o fato de $G_{n} \in \operatorname{LMT}^{k}\left(C, D_{H}\right)$.

Prova do Lema 3.7. Começamos fixando $C>1$ e $m \geq 4$. Considere um hipergrafo linear $k$-uniforme $H$ com $m$ vértices e livre de conectores. Assuma que $n p^{d_{H}} \gg n p^{D_{H}} \gg 1$.

Sabemos que existe uma ordenação $d_{H^{-}}$degenerada $\left(v_{1}, \ldots, v_{m}\right)$ dos vértices de $H$ (é fácil ver que tal ordenação existe). Tome $H_{h}=H\left[\left\{v_{1}, \ldots, v_{h}\right\}\right]$ para $1 \leq h \leq m$.

Nossa prova segue por indução em $h$, onde consideramos $n$ suficientemente grande. Provaremos que, para todo $1 \leq h \leq m$ e para todo $\varepsilon>0$, existe $\delta>0$ tal que se $G$ é um hipergrafo $k$-uniforme com $n$ vértices e $e\left(G_{n}\right)=\left(\begin{array}{l}n \\ k\end{array}\right) p$, tal que $G_{n} \in \operatorname{LMT}^{k}\left(C, D_{H}\right)$ e $G_{n} \in \mathrm{CG}_{\delta}^{k}\left(d_{H}\right)$, então

$$
|| \mathcal{I}\left(H_{h}, G_{n}\right)\left|-n^{h} p^{e\left(H_{h}\right)}\right|<\varepsilon n^{h} p^{e\left(H_{h}\right)}
$$

Fixe $\varepsilon>0$. Se $h=1$, então o resultado é trivial. Assim, suponha que $1<h \leq m$ e que o resultado é válido para $h^{\prime}<h$.

Primeiramente, mostraremos que $\left|\mathcal{I}\left(H_{h}, G_{n}\right)\right|>(1-\varepsilon) n^{h} p^{e\left(H_{h}\right)}$. Tome $\varepsilon^{\prime}=\min \{\varepsilon / 4, \varepsilon / 6 C\}$ 
e considere $\delta^{\prime}=\delta^{\prime}\left(\varepsilon^{\prime}\right)$ dada pela hipótese indutiva de modo que

$$
|| \mathcal{I}\left(H_{h-1}, G_{n}\right)\left|-n^{h-1} p^{e\left(H_{h-1}\right)}\right|<\varepsilon^{\prime} n^{h-1} p^{e\left(H_{h-1}\right)} .
$$

Fixe $\eta=\varepsilon^{\prime} / 2$ e defina $r=d_{H_{v}}\left(v_{h}\right) \leq d_{H}$. Uma aplicação do Corolário 3.16 com parâmetros $C, e\left(H_{h-1}\right), k, \eta$ e $p$ fornece o seguinte limitante na quantidade de imersões não induzidas.

$$
\left|\mathcal{I}^{\mathrm{ni}}\left(H_{h-1}, G_{n}\right)\right| \leq \eta n^{h-1} p^{e\left(H_{h-1}\right)} .
$$

Tome $\delta=\min \left\{\delta^{\prime}, \varepsilon / 8, \eta /\left(r !((k-1) !)^{r} C^{h-1-r(k-1)}\right)\right\}$. Aplicando o Corolário 3.17 com parâmetros $\delta, C$ e $m$, concluímos que

$$
\left|\mathcal{I}_{\delta-\mathrm{pol}}\left(H_{h-1}, G_{n}\right)\right| \leq \eta n^{h-1} p^{e\left(H_{h-1}\right)} .
$$

Utilizando 3.29 e 3.30 , obtemos

$$
\left|\mathcal{I}^{\mathrm{ni}}\left(H_{h-1}, G_{n}\right) \cup \mathcal{I}_{\delta-\operatorname{pol}}\left(H_{h-1}, G_{n}\right)\right| \leq 2 \eta n^{h-1} p^{e\left(H_{h-1}\right)}=\varepsilon^{\prime} n^{h-1} p^{e\left(H_{h}\right)-r} .
$$

Por (3.28), concluímos que

$$
\left(1-2 \varepsilon^{\prime}\right) n^{h-1} p^{e\left(H_{h}\right)-r}<\left|\mathcal{I}_{\delta-\text { iimpa }}^{\text {ind }}\left(H_{h-1}, G_{n}\right)\right|<\left(1+\varepsilon^{\prime}\right) n^{h-1} p^{e\left(H_{h}\right)-r} .
$$

O próximo passo é limitar por baixo o número de maneiras que podemos estender uma imersão $f^{\prime} \in \mathcal{I}_{\delta \text {-limpa }}^{\text {ind }}\left(H_{h-1}, G_{n}\right)$ para uma imersão $f \in \mathcal{I}\left(H_{h}, G_{n}\right)$. Dada tal imersão $f^{\prime}$, como $f^{\prime}$ é limpa, sabemos que $f_{k-1}^{\prime}\left(N_{H_{h}}\left(v_{h}\right)\right) \notin \mathcal{B}_{\text {est }}(\delta, r)$, i.e., ou $f^{\prime}\left(N_{H_{h}}^{\text {con }}\left(v_{h}\right)\right)$ não é estável em $G_{n}$, ou $\left|N_{G_{n}}\left(f_{k-1}^{\prime}\left(N_{H_{h}}\left(v_{h}\right)\right)\right)-n p^{r}\right|<\delta n p^{r}$.

Lembre que $H$ é linear e livre de conectores. Portanto, $N_{H_{h}}^{\text {con }}\left(v_{h}\right)$ é estável em $H_{h}$. Mas como $f^{\prime}$ é uma imersão induzida, sabemos que $f^{\prime}\left(N_{H_{h}}^{\text {con }}\left(v_{h}\right)\right)$ é estável em $G_{n}$. Desta forma, concluímos que

$$
\left|N_{G_{n}}\left(f_{k-1}^{\prime}\left(N_{H_{h}}\left(v_{h}\right)\right)\right)-n p^{r}\right|<\delta n p^{r}
$$

Para obter uma extensão $f \in \mathcal{I}\left(H_{h}, G_{n}\right)$ de $f^{\prime} \in \mathcal{I}\left(H_{h-1}, G_{n}\right)$ é preciso selecionar $f\left(v_{h}\right)$ em $N_{G_{n}}\left(f_{k-1}^{\prime}\left(N_{H_{h}}\left(v_{h}\right)\right)\right) \backslash f^{\prime}\left(V\left(H_{h-1}\right)\right)$. Assim, a quantidade de tais extensões é limitada por baixo por

$$
\left|N_{G_{n}}\left(f_{k-1}^{\prime}\left(N_{H_{h}}\left(v_{h}\right)\right)\right) \backslash f^{\prime}\left(V\left(H_{h-1}\right)\right)\right| \geq(1-\delta) n p^{r}-(h-1) \geq(1-2 \delta) n p^{r},
$$

onde a primeira desigualdade é devido a (3.32) e a última segue do fato de $n p^{r} \gg 1$. Por (3.31) 
e 3.33 , temos

$$
\begin{aligned}
\left|\mathcal{I}\left(H_{h}, G_{n}\right)\right| & \geq\left|\mathcal{I}_{\delta \text {-limpa }}^{\text {ind }}\left(H_{h}, G_{n}\right)\right| \\
& \geq\left|\mathcal{I}_{\delta-\text { limpa }}^{\text {ind }}\left(H_{h-1}, G_{n}\right)\right|\left|N_{G_{n}}\left(f_{k-1}^{\prime}\left(N_{H_{h}}\left(v_{h}\right)\right)\right) \backslash f^{\prime}\left(V\left(H_{h-1}\right)\right)\right| \\
& >\left(1-2 \varepsilon^{\prime}\right)(1-2 \delta) n^{h-1} p^{e\left(H_{h}\right)-r} n p^{r} \\
& \geq(1-\varepsilon) n^{h} p^{e\left(H_{h}\right)},
\end{aligned}
$$

onde a última desigualdade segue da escolha de $\varepsilon^{\prime}$ e $\delta$.

Precisamos mostrar que $\left|\mathcal{I}\left(H_{h}, G_{n}\right)\right|<(1+\varepsilon) n^{h} p^{e\left(H_{h}\right)}$. Fixe uma imersão $f^{\prime} \in \mathcal{I}\left(H_{h-1}, G_{n}\right)$. Deixe-nos considerar primeiramente o caso $f^{\prime} \in \mathcal{I}_{\delta \text {-limpa }}^{\text {ind }}\left(H_{h-1}, G_{n}\right)$. Note que a quantidade de extensões de $f^{\prime}$ para imersões de $H_{h}$ em $G_{n}$ é no máximo $\left|N_{G_{n}}\left(f_{k-1}^{\prime}\left(N_{H_{h}}\left(v_{h}\right)\right)\right)\right|$. Portanto, por 3.31 e 3.32, a quantidade de imersões de $H_{h}$ em $G_{n}$, onde a imersão de $H_{h-1}$ é limpa e induzida é no máximo

$$
\begin{aligned}
\left|\mathcal{I}_{\delta-\text { limpa }}^{\text {ind }}\left(H_{h-1}, G_{n}\right)\right|\left|N_{G_{n}}\left(f_{k-1}^{\prime}\left(N_{H_{h}}\left(v_{h}\right)\right)\right)\right| & \leq\left(1+\varepsilon^{\prime}\right) n^{h-1} p^{e\left(H_{h}\right)-r}(1+\delta) n p^{r} \\
& \leq(1+\varepsilon / 2) n^{h} p^{e\left(H_{h}\right)},
\end{aligned}
$$

onde a última desigualdade segue da escolha de $\varepsilon^{\prime}$ e $\delta$.

Suponha agora que $f^{\prime} \in\left\{\mathcal{I}\left(H_{h-1}, G_{n}\right) \backslash \mathcal{I}_{\delta-\text { limpa }}^{\text {ind }}\left(H_{h-1}, G_{n}\right)\right\}$. Por 3.28 e 3.31 , temos

$$
\left|\mathcal{I}\left(H_{h-1}, G_{n}\right) \backslash \mathcal{I}_{\delta-\text { impa }}^{\text {ind }}\left(H_{h-1}, G_{n}\right)\right| \leq 3 \varepsilon^{\prime} n^{h-1} p^{e\left(H_{h}-r\right)} .
$$

Mas como $r=d_{H_{h}}\left(v_{h}\right) \leq d_{H} \leq D_{H}$ e $G_{n} \in \operatorname{LMT}^{k}\left(C, D_{H}\right)$, sabemos que cada imersão $f^{\prime}$ de $H_{h-1}$ em $G_{n}$ pode ser estendida a no máximo $C n p^{r}$ imersões $f \in \mathcal{I}\left(H_{h}, G_{n}\right)$. De fato, para ver isso, precisamos somente fazer uso da propriedade $\operatorname{LMT}^{k}\left(C, D_{H}\right)$ na seguinte família de conjuntos de $k-1$ vértices:

$$
\left\{f^{\prime}\left(S_{1}\right), \ldots, f^{\prime}\left(S_{\left|N_{H_{h}}\left(v_{h}\right)\right|}\right)\right\}
$$

onde $\left\{S_{1}, S_{2}, \ldots, S_{\left|N_{H_{h}}\left(v_{h}\right)\right|}\right\}$ é a vizinhança de $v_{h}$ em $H_{h}$. Esse fato, juntamente com (3.36), implica que a quantidade de imersões de $H_{h}$ em $G_{n}$, onde as imersões de $H_{h-1}$ não são $\delta$-limpas ou não são induzidas é no máximo $\left(3 \varepsilon^{\prime} C\right) n^{h} p^{e\left(H_{h}\right)} \leq(\varepsilon / 2) n^{h} p^{e\left(H_{h}\right)}$. Colocando isso juntamente com 3.35, obtemos $\left|\mathcal{I}\left(H_{h}, G_{n}\right)\right|<(1+\varepsilon) n^{h} p^{e\left(H_{h}\right)}$, finalizando a prova. 


\section{Capítulo 4}

\section{Números de Ramsey para grafos bipartidos}

Para grafos $G_{1}, G_{2}, \ldots, G_{r}$, o número de Ramsey $R\left(G_{1}, G_{2}, \ldots, G_{r}\right)$ é o menor inteiro positivo $n$ tal que, se as arestas de um grafo completo $K_{n}$ são particionadas em $r$ classes de cores distintas, fornecendo $r$ grafos $H_{1}, H_{2}, \ldots, H_{r}$, então pelo menos um dos grafos $H_{i}(1 \leq i \leq r)$ contém um subgrafo isomorfo a $G_{i}$. A existência de tal inteiro positivo segue do clássico Teorema de Ramsey. O número $R\left(G_{1}, G_{2}, \ldots, G_{r}\right)$ é chamado de número de Ramsey de ordem $r$ para os grafos $G_{1}, G_{2}, \ldots, G_{r}$. Determinar $R\left(G_{1}, G_{2}, \ldots, G_{r}\right)$ para grafos gerais se mostrou ser um problema difícil (veja, e.g., [28] ou [47]).

Para números de Ramsey de ordem dois, um conhecido teorema de Gerencsér e Gyárfás [24] afirma que

$$
R\left(P_{n}, P_{n}\right)=\left\lfloor\frac{3 n-2}{2}\right\rfloor,
$$

onde $P_{n}$ denota o caminho com $n \geq 2$ vértices. Em [32], árvores mais gerais foram consideradas. Para uma árvore $T$, escrevemos $t_{1}$ e $t_{2}$, com $t_{2} \geq t_{1}$, para os tamanhos das classes de vértices de $T$, quando vista como um grafo bipartido. Note que $R(T, T) \geq 2 t_{1}+t_{2}-1$, dado que a seguinte coloração das arestas de $K_{2 t_{1}+t_{2}-2}$ não possui cópia monocromática de $T$. Particione os vértices em duas classes $V_{1}$ e $V_{2}$ tais que $\left|V_{1}\right|=t_{1}-1$ e $\left|V_{2}\right|=t_{1}+t_{2}-1$, então use a cor 'vermelha' para todas as arestas dentro das duas classes e use a cor 'azul' para todas as arestas entre as classes. Ademais, uma coloração semelhante das arestas de $K_{2 t_{2}-2}$ com duas classes, ambas de tamanho $t_{2}-1$, mostra que $R(T, T) \geq 2 t_{2}-1$, onde aqui a cor que colocamos nas arestas que estão dentro das classes com $t_{1}$ vértices é arbitrária. Assim,

$$
R(T, T) \geq \max \left\{2 t_{1}+t_{2}, 2 t_{2}\right\}-1 \text {. }
$$

Haxell, Łuczak e Tingley [32] provaram que o limitante em 4.1] é assintoticamente correto para árvores $T$ com $\Delta(T)=o\left(t_{2}\right)$. Apresentamos uma versão desse resultado para certos grafos bipartidos. Dizemos que um grafo $H=\left(W, E_{H}\right)$ possui largura de banda no máximo $b$, se existe uma rotulação dos vértices de $H$ com os números $1, \ldots, n$, de modo que, para 
cada aresta $i j \in E_{H}$, temos $|i-j| \leq b$. Concentramos nossa atenção na seguinte classe de grafos bipartidos.

Definição 4.1. Um grafo $H$ é chamado de $(\beta, \Delta)$-bigrafo se é bipartido, possui largura de banda no máximo $\beta\left|V_{H}\right|$ e seu grau máximo não é maior que $\Delta$. Ademais, dizemos que $H$ é um $(\beta, \Delta)$-bigrafo balanceado se existe uma 2-coloração própria $\chi: V_{H} \rightarrow[2]$ tal que ||$\chi^{-1}(1)|-| \chi^{-1}(2)|| \leq \beta\left|\chi^{-1}(2)\right|$.

Por exemplo, foi provado em [7] que grafos planares suficientemente grandes com grau máximo não maior que $\Delta$ são $(\beta, \Delta)$-bigrafos para qualquer $\beta>0$. O primeiro teorema deste capítulo é um resultado análogo ao resultado em [32], para $(\beta, \Delta)$-bigrafos.

Teorema 4.2 (Teorema 2-Ramsey). Para todo $\gamma>0$ e número natural $\Delta$, existem uma constante $\beta>0$ e um número natural $n_{0}$ tais que, para todo $(\beta, \Delta)$-bigrafo $H$ com $n \geq n_{0}$ vértices que contém uma 2 -coloração própria $\chi: V_{H} \rightarrow[2]$, onde $t_{1}=\left|\chi^{-1}(1)\right|$ e $t_{2}=\left|\chi^{-1}(2)\right|$, com $t_{1} \leq t_{2}$, temos

$$
R(H, H) \leq(1+\gamma) \max \left\{2 t_{1}+t_{2}, 2 t_{2}\right\}
$$

Para resultados relacionados com números de Ramsey de grafos com número cromático alto e largura de banda sublinear, veja o trabalho de Allen, Brightwell e Skokan [1].

Menos ainda é conhecido sobre números de Ramsey de ordem três. Seja $T$ uma árvore e considere $t_{1}$ e $t_{2}$, com $t_{2} \geq t_{1}$, os tamanhos das classes de vértices de $T$ quando vista como um grafo bipartido. A seguinte construção dá um limitante inferior para $R(T, T, T)$. Particione os vértices de $T$ em quatro classes, uma classe especial $V_{0}$ com $\left|V_{0}\right|=t_{1}$ e três classes $V_{1}, V_{2}$ e $V_{3}$ de tamanho $t_{2}-1$. A cor para as arestas que estão dentro de $V_{0}$ é arbitrária. Use a cor $i$ dentro da classe $V_{i}$ e a cor $i$ entre as classes $V_{i}$ e $V_{0}$ para $1 \leq i \leq 3$. Finalmente, use a cor $k \in[3] \backslash\{i, j\}$ para arestas entre as classes $V_{i}$ e $V_{j}$ para $1 \leq i<j \leq 3$. É fácil verificar que essa coloração não gera nenhuma cópia monocromática de T. Assim,

$$
R(T, T, T) \geq t_{1}+3 t_{2}-2
$$

Estabelecendo a validade de uma conjectura de Faudree e Schelp [17], foi provado em [29] que essa construção é ótima para caminhos longos, i.e., para $n$ suficientemente grande, temos que

$$
R\left(P_{n}, P_{n}, P_{n}\right)=\left\{\begin{array}{l}
2 n-1 \text { para } n \text { ímpar } \\
2 n-2 \text { para } n \text { par. }
\end{array}\right.
$$

Assintoticamente, esse resultado foi provado, independentemente, por Figaj e Łuczak [18]. Benevides e Skokan [3] provaram que $R\left(C_{n}, C_{n}, C_{n}\right)=2 n$ para $n$ par suficientemente grande. O segundo teorema que apresentamos neste capítulo estende assintoticamente esses resultados para $(\beta, \Delta)$-bigrafos.

Teorema 4.3 (Teorema 3-Ramsey). Para todo $\gamma>0$ e todo número natural $\Delta$, existe uma constante $\beta>0$ e um número natural $n_{0}$ tal que, para todo $(\beta, \Delta)$-bigrafo balanceado $H$ com 
$n \geq n_{0}$ vértices, temos que

$$
R(H, H, H) \leq(2+\gamma) n
$$

Em particular, os Teoremas 4.2 e 4.3 fornecem assintoticamente os números de Ramsey de ordem dois e três. Uma grade bi-dimensional $G_{a, b}$ é o grafo com conjunto de vértices $V=[a] \times[b]$ tal que existe uma aresta entre dois vértices se e somente se eles são iguais em uma coordenada e consecutivos na outra. Note que qualquer grade $G_{a, b}$ satisfaz $\Delta(G) \leq$ 4 e possui largura de banda no máximo $\sqrt{a b}$. Logo, $G_{a, b}$ é um $(\beta, 4)$-bigrafo balanceado para qualquer $\beta>0$ fixo, desde que $a b$ seja suficientemente grande. Consequentemente, os Teoremas 4.2 e 4.3 combinados com 4.1) e 4.2 implicam o seguinte corolário.

Corolário 4.4. Para grades $G_{a, b}$, temos

$$
\begin{aligned}
R\left(G_{a, b}, G_{a, b}\right) & =(3 / 2+o(1)) a b, \\
R\left(G_{a, b}, G_{a, b}, G_{a, b}\right) & =(2+o(1)) a b,
\end{aligned}
$$

onde o(1) tende a 0 quando ab tende a infinito.

Observe que valores similares valem para grafos bipartidos planares com grau limitado e para grades de dimensão maior.

Apresentamos neste capítulo a prova detalhada do Teorema 4.3. Primeiramente, na Seção 4.1. é dada uma visão geral da prova do Teorema 4.3. Na Seção 4.2, fornecemos as ferramentas necessárias para provar o Teorema 4.3, que é provado em todos os seus detalhes na Seção 4.3. A prova do Teorema 4.2 é muito similar à prova do Teorema 4.3, de modo que apresentamos somente uma ideia geral de sua prova, na Seção 4.4 .

\subsection{Visão geral da prova do Teorema 3-Ramsey}

Aqui discutimos as principais ideias envolvidas na prova do Teorema 4.3 , tentando ao máximo evitar os detalhes técnicos, que são postergados até a Seção 4.3 . A prova faz uso do método da regularidade, que é explicado na Seção 4.2.1. Dividimos a prova em duas partes.

A primeira parte da prova segue o mesmo padrão da prova de Figaj e Łuczak [18] para o caso em que $H$ é um caminho. A saber, aplicamos a versão multicolorida do Lema de Regularidade de Szemerédi [53], obtendo uma partição dos vértices de $K_{N}$, com as arestas coloridas com 3 cores, onde $N=(2+\gamma) n$, tal que essa partição possui um grafo reduzido $R$ correspondente suficientemente denso. Cada aresta do grafo reduzido $R$ herda uma das cores que aparece mais vezes no par correspondente em $K_{N}$. Aplicando o Lema 8 de [18], obtemos uma árvore monocromática $T$ no grafo reduzido $R$ que contém um emparelhamento $M$ cobrindo quase metade dos vértices de $R$.

Voltando nossa atenção novamente ao grafo completo $K_{N}$ e à 3-coloração de suas arestas, denotamos por $G_{T}$ o subgrafo de $K_{N}$, cujos vértices estão contidos nas classes representadas 
pelos vértices de $T$, e cujas arestas estão entre pares representados pelas arestas de $T$ e possuem a mesma cor das arestas de $T$. Assim, $G_{T}$ é um subgrafo monocromático de $K_{N}$ tal que os pares regulares estão dispostos em uma estrutura que espelha a estrutura de T. Ademais, a densidade de arestas desses pares é pelo menos $1 / 3$. Por fim, localizamos subgrafos super-regulares 'quase-geradores' nos pares de $G_{T}$ representados por arestas de $M$, e denotamos por $G_{M} \subset G_{T}$ o subgrafo formado pela união desses pares. Completamos assim a primeira parte da prova.

Para entender a motivação para a segunda parte da prova, tenha em mente que nosso objetivo é realizar a imersão de $H$ em $G_{T}$. Note que $G_{M}$ possui vértices suficientes para acomodar todos os vértices de $H$. De fato, a maioria dos vértices de $H$ serão mapeados em $G_{M}$ e iremos precisar utilizar somente algumas partes de $G_{T} \backslash G_{M}$, pois precisaremos conectar as várias partes de $H$ imersas em $G_{M}$. Deixe-nos explicar isso de forma mais precisa, assumindo, por ora, que $H$ é simplesmente um caminho. Seja $m$ um inteiro que é somente um pouco menor que o tamanho das classes de $G_{T}$ (que assumimos serem todas do mesmo tamanho). Aplicando o Lema de Explosão de Komlós, Sárközy e Szemerédi [41], realizamos a imersão dos primeiros $m$ vértices de cada uma das duas classes de cor de $H$ no par super-regular, representado pela primeira aresta do emparelhamento $M$ (lembre que $H$ é balanceado).

Para conseguirmos 'alcançar' o próximo par super-regular em $G_{M}$, onde podemos realizar a imersão dos próximos $m+m$ vértices de $H$, precisamos fazer uso do fato de os vértices representando esses dois pares serem conectados por um caminho em $T$. Esse caminho se traduz em uma sequência de pares regulares em $G_{T}$, de modo que, em cada um deles, fazemos a imersão de uma aresta intermediária de $H$, 'caminhando' assim para o próximo par superregular em $G_{M}$. Desta forma, utilizamos somente poucas arestas de pares regulares que estão em $G_{T} \backslash G_{M}$, os mantendo regulares por todo o caminho e deixando um pouco de espaço nos pares super-regulares de $G_{M}$, o que nos permite caminhar por eles novamente. Observamos que faremos uso de um corolário do Lema de Explosão, que encapsula toda a ideia descrita acima (veja Lema 4.7).

No parágrafo anterior, consideramos $H$ como sendo um caminho. Portanto, a tarefa para essa segunda parte da prova é reestruturar o $(\beta, \Delta)$-bigrafo balanceado $H$ de modo que ele se comporte de forma semelhante a um caminho na abordagem de imersão descrita acima. Aqui, dois problemas podem ocorrer:

- Problema 1: Suponha, por exemplo, que $H$ é um grafo consistindo de um caminho com conjunto de vértices $[n]$, contendo algumas arestas adicionais entre os vértices cujos rótulos diferem de no máximo $\beta n$ e possuem paridade diferente (pois $H$ deve ser bipartido). Para tal grafo, 'realizar as conexões' como explicado anteriormente é um pouco mais complicado. Suponha, por exemplo, que temos uma cadeia de pares regulares $\left(V_{i}, V_{i+1}\right)$ em $G_{T}$ para $i=1, \ldots, 4$ e queremos utilizá-la para 'caminhar' com $H$, da classe $V_{1}$ até a classe $V_{5}$. Não podemos simplesmente mapear o vértice 1 
à classe $V_{1}$, o vértice 2 à classe $V_{2}$ e assim por diante, até mapear o vértice 5 à classe $V_{5}$, pois talvez $\{2,5\}$ seja uma aresta de $H$, mas $\left(V_{2}, V_{5}\right)$ não forma um par regular em $G_{T}$, de modo que não poderíamos aplicar o Lema de Explosão nesse ponto.

Solução: Caminhar mais lentamente: mapeie o vértice 1 à classe $V_{1}$, então, com os vértices $2,3, \ldots, \beta n+1$, alterne entre $V_{2}$ e $V_{3}$. Os próximos $\beta n$ vértices continuam o padrão de zigue-zague entre $V_{3}$ e $V_{4}$ até o penúltimo vértice, de modo que o último vértice é mapeado em $V_{5}$. Por que razão essa é uma boa estratégia? Considere, por exemplo, o último vértice $y$ que foi mapeado em $V_{5}$. Devido à condição de largura de banda (a vizinhança de todo vértice $i$ está contida em $[i-\beta n, i+\beta n]$ ), todos os potenciais vizinhos de $y$ foram mapeados em $V_{3}$ ou $V_{4}$, e, devido à condição de paridade, todos eles devem estar em $V_{4}$. Mas isso é muito bom, pois $\left(V_{4}, V_{5}\right)$ é um par regular.

- Problema 2: Por definição, $H$ possui uma 2-coloração de seus vértices que utiliza as duas cores aproximadamente a mesma quantidade de vezes, no total. Porém, isso não necessariamente vale localmente. Isto é, dentre os primeiros $m+m$ vértices de $H$, podem existir muitos vértices com a cor 1 e poucos vértices com a cor 2 , o que significa que nossa abordagem para mapeá-los em pares super-regulares com duas classes de mesmo tamanho irá falhar.

Solução: Balancear $H$ de modo que as cores fiquem melhor distribuídas. Utilizamos uma ordenação de $H$ com largura de banda no máximo $\beta n$ e 'fatiamos' $H$ em pequenos blocos de tamanho $\xi n$, onde $\beta n \ll \xi n \ll m$. Então, não é difícil ver que podemos obter uma nova ordenação dos vértices de $H$ mudando a ordem em que os blocos aparecem, de modo que em cada intervalo de blocos que somam $m$ vértices consecutivos, as duas cores são balanceadas, a menos de $\xi n$ vértices. Podemos assim mapear os blocos compostos por esses intervalos em pares super-regulares de $G_{M}$, o que nos permite aplicar o Lema de Explosão nos pares super-regulares.

Com certeza, os dois problemas citados podem ocorrer ao mesmo tempo, mas mostraremos que é possível combinar as soluções para os dois problemas. Portanto, podemos, similarmente ao exemplo de caminhos que fornecemos anteriormente, realizar a imersão de $H$ em $G_{T}$, finalizando a prova.

\subsection{Resultados auxiliares}

O principal objetivo desta seção é apresentar as ferramentas necessárias para a prova do Teorema 4.3. Um dos principais resultados utilizados na prova é o Lema de Regularidade de Szemerédi [53]. Discutimos o Método da Regularidade, na Seção 4.2.1. Nas Seções 4.2.2 e 4.2.3 fornecemos alguns resultados que nos permitirão aplicar com sucesso o método da regularidade para provar o Teorema 4.3 . 


\subsubsection{Método da Regularidade}

Introduzimos aqui as ferramentas necessárias para aplicar com sucesso o Método da Regularidade no contexto que precisamos. Mais especificamente, apresentamos uma versão do Lema de Regularidade de Szemerédi para 3-colorações de arestas e um corolário do lema de contagem (Lema de Explosão) de Komlós, Sárközy e Szemerédi [41], apropriado para nossos propósitos.

Um grafo bipartido $G=(A, B ; E)$ é chamado de $\varepsilon$-regular se, para todo $X \subset A, Y \subset B$ $\operatorname{com}|X|>\varepsilon|A|$ e $|Y|>\varepsilon|B|$ temos

$$
\left|d_{G}(X, Y)-d_{G}(A, B)\right|<\varepsilon .
$$

Ademais, dizemos que um grafo bipartido $G=(A, B ; E)$ é $(\varepsilon, d)$-regular se $G$ é $\varepsilon$-regular com $d_{G}(A, B) \geq d$. Ademais, se $\operatorname{deg}_{G}(a)>d|B|$ para todo $a \in A$, e $\operatorname{deg}_{G}(b)>d|A|$ para todo $b \in B$, então dizemos que $G$ é $(\varepsilon, d)$-super-regular .

Dado um grafo $G=(V, E)$, uma partição $\left(V_{i}\right)_{i \in[s]}$ de $V$ é dita $(\varepsilon, d)$-regular (resp. superregular) em um grafo $R$ com conjunto de vértices contido em $[s]$ se o subgrafo bipartido de $G$ induzido pelo par $\left\{V_{i}, V_{j}\right\}$ é $(\varepsilon, d)$-regular (resp. super-regular) sempre que $i j \in E(R)$. Dizemos que $R$ é um grafo $(\varepsilon, d)$-reduzido de $\left(V_{i}\right)_{i \in[s]}$ (ou de $G$ ).

A prova do Teorema 4.3 é baseada na seguinte versão do Lema de Regularidade de Szemerédi [53] para 3-colorações de arestas.

Lema 4.5 (Lema de Regularidade). Para todo $\varepsilon>0$ e todo inteiro $k_{0}>0$ existe um inteiro positivo $K_{0}\left(\varepsilon, k_{0}\right)$ tal que, para $n \geq K_{0}$, o seguinte vale. Para todos os grafos $G_{1}, G_{2}$ e $G_{3}$ com $V\left(G_{1}\right)=V\left(G_{2}\right)=V\left(G_{3}\right)=V$, onde $|V|=n$, existe uma partição de $V$ em $k+1$ classes

$$
V=V_{0} \cup V_{1} \cup V_{2} \cup \cdots \cup V_{k}
$$

tal que

- $\quad k_{0} \leq k \leq K_{0}$

- $\left|V_{1}\right|=\left|V_{2}\right|=\cdots=\left|V_{k}\right|$

- $\left|V_{0}\right|<\varepsilon n$,

- $\quad$ pelo menos $(1-\varepsilon)\left(\begin{array}{l}k \\ 2\end{array}\right)$ pares $\left\{V_{i}, V_{j}\right\}$ são $\varepsilon$-regulares em $G_{1}, G_{2}$ e $G_{3}$.

Para um estudo aprofundado acerca do Lema de Regularidade e suas aplicações, veja [40] e [43]. Um componente chave do método da regularidade é o Lema de Explosão [41] (veja também [42, 49, 51] para provas alternativas). Esse lema garante que subgrafos geradores bipartidos de grau limitado podem ser imersos em pares super-regulares. Na verdade, a afirmação é mais geral, e permite a imersão de grafos $r$-cromáticos na união de $r$ classes de vértices que formam $\left(\begin{array}{l}r \\ 2\end{array}\right)$ pares super-regulares. 
Aqui utilizamos uma versão do Lema de Explosão que nos permite realizar a imersão de grafos $H$ com grau limitado em um grafo $G$, quando $G$ e $H$ possuem partições 'compatíveis', no sentido explicado na Definição 4.6 dada a seguir. Em nossa prova, vamos realizar a imersão de $H$ por partes, considerando uma partição de um subgrafo monocromático $G$ de $K_{N}$, onde o grafo reduzido correspondente contém uma árvore $T$ contendo um emparelhamento $M$ 'grande', onde os grafos bipartidos de $G$ que correspondem às arestas de $M$ são pares superregulares.

Definição 4.6. Seja $H=\left(W, E_{H}\right)$ um grafo. Considere uma árvore $T=\left([s], E_{T}\right)$ e um subgrafo $M=\left([s], E_{M}\right)$ de $T$, onde $E_{M}$ é um emparelhamento. Dada uma partição $\left(W_{i}\right)_{i \in[s]}$, seja $U_{i}$ o conjunto de vértices em $W_{i}$ com vizinhança em algum $W_{j}$, onde ij $\in E_{T} \backslash E_{M}$. Defina $U:=\bigcup U_{i}$ e $U_{i}^{\prime}:=N_{H}(U) \cap\left(W_{i} \backslash U\right)$.

Dizemos que $\left(W_{i}\right)_{i \in[s]}$ é $(\varepsilon, T, M)$-compatível com uma partição $\left(V_{i}\right)_{i \in[s]}$ dos vértices de um grafo $G=(V, E)$ se o seguinte vale.

(i) $x y \in E_{H}$ para $x \in W_{i}$ e $y \in W_{j}$ implica que ij $\in E_{T}$. Em outras palavras, o mapeamento de $W$ para $[s]$ dado pela partição $\left(W_{i}\right)_{i \in[s]}$ é um homomorfismo de $H$ em $T$,

(ii) $\left|W_{i}\right| \leq\left|V_{i}\right|$ para todo $i \in[s]$,

(iii) $\left|U_{i}\right| \leq \varepsilon\left|V_{i}\right|$ para todo $i \in[s]$,

(iv) $\left|U_{i}^{\prime}\right|,\left|U_{j}^{\prime}\right| \leq \varepsilon \min \left\{\left|V_{i}\right|,\left|V_{j}\right|\right\}$ para toda aresta $i j \in E_{M}$.

O seguinte corolário do Lema de Explosão afirma que, seguindo a configuração da Definição 4.6, grafos $H$ com grau limitado podem ser imersos em $G$, se $G$ admite uma partição suficientemente regular em $T$ e super-regular em $M$.

Lema 4.7 (Lema de Explosão [5, 6]). Para todo $d>0$ e todo $\Delta>0$, existe $\varepsilon=\varepsilon(d, \Delta)>0$ tal que o seguinte vale. Seja $G=(V, E)$ um grafo com $N$ vértices que possui uma partição $\left(V_{i}\right)_{i \in[s]}$ de $V$ com um grafo $(\varepsilon, d)$-reduzido $T$ com conjunto de vértices $[s]$ que é $(\varepsilon, d)$-superregular em um subgrafo $M \subset T$. Ademais, seja $H=\left(W, E_{H}\right)$ um grafo com $n$ vértices, com grau máximo $\Delta(H) \leq \Delta$ e $n \leq N$, tal que $H$ possui uma partição $\left(W_{i}\right)_{i \in[s]}$ de seus vértices que é $(\varepsilon, T, M)$-compatível com $\left(V_{i}\right)_{i \in[s]}$. Então $H \subset G$.

Encerramos esta seção com dois fatos simples, que seguem das definições de pares regulares e super-regulares (para provas, veja, respectivamente, [27] e [45]).

Afirmativa 4.8. Seja $B=\left(V_{1}, V_{2} ; E\right)$ um grafo bipartido e-regular e tome $V_{1}^{\prime} \subset V_{1}$ e $V_{2}^{\prime} \subset V_{2}$ com $\left|V_{1}^{\prime}\right| \geq \alpha\left|V_{1}\right|$ e $\left|V_{2}^{\prime}\right| \geq \alpha\left|V_{2}\right|$ para algum $\alpha>\varepsilon$. Então $B^{\prime}=\left(V_{1}^{\prime}, V_{2}^{\prime} ; E_{B}\left(V_{1}^{\prime}, V_{2}^{\prime}\right)\right)$ é um grafo $\varepsilon^{\prime}$-regular com $\varepsilon^{\prime}=\max \{\varepsilon / \alpha, 2 \varepsilon\}$ tal que $\left|d_{B}\left(V_{1}, V_{2}\right)-d_{B^{\prime}}\left(V_{1}^{\prime}, V_{2}^{\prime}\right)\right|<\varepsilon$. 
Afirmativa 4.9. Considere um grafo $G=(V, E)$ com uma partição $(\varepsilon, d)$-regular de seus vértices $\left(V_{i}\right)_{i \in[s]}$ com $\left|V_{i}\right|=m$ para $1 \leq i \leq s$ e seja $T$ a árvore com conjunto de vértices $[s]$ contida no grafo $(\varepsilon, d)$-reduzido correspondente. Seja $E_{M}$ um emparelhamento contido em T. Então, para cada vértice $i$ coberto por $E_{M}$, o conjunto associado $V_{i}$ em $G$ contém um subconjunto $V_{i}^{\prime}$ de tamanho $(1-\varepsilon r) m$, para qualquer $r \geq 1$, tal que, para cada aresta ij de $E_{M}$, o grafo bipartido $G^{\prime}=\left(V_{i}^{\prime}, V_{j}^{\prime} ; E(G)\left(V_{i}^{\prime}, V_{j}^{\prime}\right)\right)$ é $(\varepsilon /(1-\varepsilon r), d-(1+r) \varepsilon)$-super-regular.

\subsubsection{Explosão regular de uma árvore}

Nesta seção, mostramos que, para qualquer 3-coloração de $E\left(K_{N}\right)$, existe um subgrafo monocromático de $K_{N}$, denso e regular, que possui algumas propriedades estruturais interessantes, de modo que será possível realizar a imersão de $H$ nesse subgrafo. Aqui, a noção de emparelhamento conexo (originada em [46], veja também [18, 29, 30, 31]) tem um papel fundamental. Um emparelhamento conexo em um grafo $R$ é um emparelhamento $E_{M}$ tal que as arestas de $E_{M}$ estão em um mesmo componente conexo de $R$. Um resultado importante afirma que, em toda 3-coloração das arestas de um grafo 'quase completo', existe um emparelhamento $E_{M}$ que cobre quase metade dos vértices do grafo, onde $E_{M}$ está contido em uma árvore monocromática.

Lema 4.10 (Figaj-Łuczak [18]). Para todo $\delta>0$, existem $\varepsilon_{0}>0$ e um natural $k_{0}$ tal que, para todo $\varepsilon \leq \varepsilon_{0}$ e $k \geq k_{0}$ e, para toda 3 -coloração das arestas de um grafo $R$ com $k$ vértices e densidade pelo menos $1-\varepsilon$, existe um emparelhamento $E_{M}$ com pelo menos $(1-\delta) k / 4$ arestas em $R$ que está contido em uma árvore monocromática $T \subset R$.

Esse lema pode ser visto em uma forma estrutural mais forte em [29]. De fato, é provado que, ou existe um emparelhamento conexo monocromático que cobre mais da metade dos vértices, ou o grafo $R$ é próximo a um de dois casos extremais, de modo que, em ambos os casos extremais, existe um emparelhamento $E_{M}$ de tamanho pelo menos $(1-\delta)|V(R)| / 4$. Iremos também fazer uso da seguinte afirmativa.

Afirmativa 4.11. Se uma árvore $T$ contém um emparelhamento $E_{M}$ com $\ell$ arestas, então os vértices cobertos pelo emparelhamento podem ser rotulados com $x_{1}, y_{1}, \ldots, x_{\ell}, y_{\ell}$ de modo que $E_{M}=\left\{x_{i} y_{i}: i=1, \ldots, \ell\right\}$ e os vértices $x_{i}$ e $x_{j}$ estão a uma distância par em $T$, para todo $1 \leq i<j \leq \ell$.

De fato, considere uma 2-coloração própria $\chi: V(T) \rightarrow[2]$. Rotule com $x_{i}$ as extremidades que estão em $\chi^{-1}(1)$ e rotule com $y_{i}$ as extremidades que estão em $\chi^{-1}(2)$. Claramente, a distância em $T$ entre $x_{i}$ e $x_{j}$ é par, pois pertencem a uma mesma classe da bipartição.

Dada uma coloração $c: E\left(K_{N}\right) \rightarrow[3]$, denotamos por $K_{N}^{1}$ o subgrafo gerador de $K_{N}$ tal que $i j \in E\left(K_{N}^{1}\right)$ se e somente se $c(i j)=1$.

Lema 4.12. Para todo $\gamma>0$, existe $\varepsilon_{0}$ tal que, para todo $\varepsilon \leq \varepsilon_{0}$, existe um natural $K_{0}$ tal que, para todo $n \geq K_{0} /(2+\gamma)$ e para toda coloração $c: E\left(K_{N}\right) \rightarrow[3]$, onde $N=(2+\gamma) n$, 
vale o seguinte: existe uma cor (digamos que seja a cor 1 ), inteiros $\ell, \ell^{\prime}, k$ com $\ell, \ell^{\prime} \leq k \leq K_{0}$ e $\ell \geq(1-\gamma / 4) k / 4$, uma árvore $T$ com conjunto de vértices $\left\{x_{1}, \ldots, x_{\ell}, y_{1}, \ldots, y_{\ell}, z_{1}, \ldots, z_{\ell^{\prime}}\right\}$ contendo um emparelhamento $E_{M}=\left\{x_{i} y_{i}: i=1, \ldots, \ell\right\}$ com uma distância par entre quaisquer $x_{i}$ e $x_{j}$, tal que existe uma partição $\left(V_{i}\right)_{0 \leq i \leq k}$ de $V_{K_{N}^{1}}$ que é $(\varepsilon, 1 / 3)$-regular em $T$, com $\left|V_{1}\right|=\ldots=\left|V_{k}\right| \geq(1-\varepsilon) N / k$.

O Lema 4.12 faz uso do Lema de Regularidade (Lema 4.5) e do Lema 4.10 para encontrar uma estrutura monocromática no grafo $K_{N}$ que será útil na prova do Teorema 4.3 . Na Figura 4.1 é possível visualizar melhor a estrutura do subgrafo monocromático de $K_{N}$ induzido pelas classes de vértices que representam os vértices da árvore $T$.

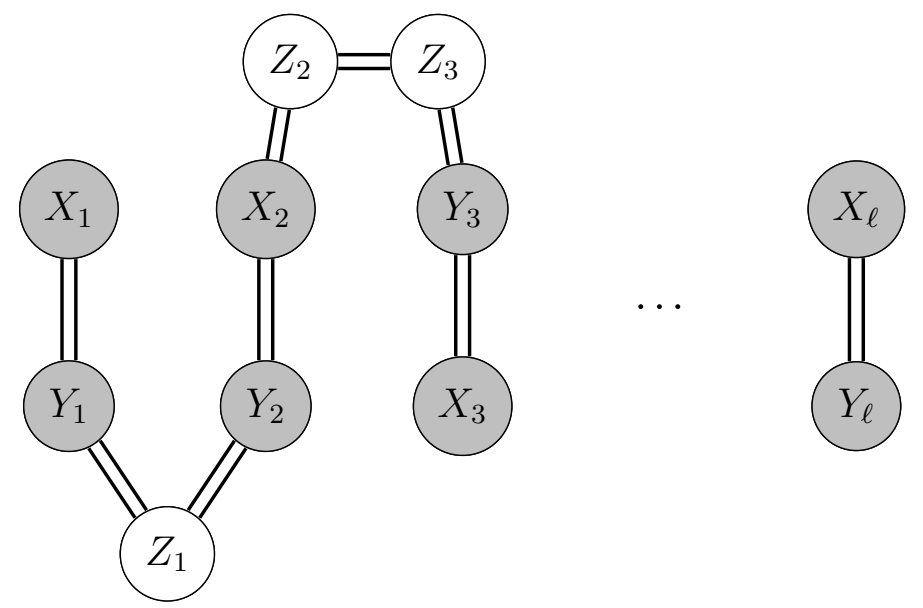

Figura 4.1: Subgrafo monocromático de $K_{N}$ induzido pelas classes de vértices que representam os vértices da árvore $T$ obtida no Lema 4.12. Classes formando pares regulares são conectadas por duas linhas paralelas.

Demonstração do Lema 4.12. Fixe $\gamma>0$ e defina $\delta=\gamma / 4$. Sejam $\varepsilon_{0}$ e $k_{0}$ as constantes obtidas pelo Lema 4.10 aplicado $\operatorname{com} \delta$. Fixe $\varepsilon \leq \varepsilon_{0}$ e seja $K_{0}$ obtido por uma aplicação do Lema de Regularidade (Lema 4.5) com parâmetros $\varepsilon$ e $k_{0}$. Por fim, fixe $N=(2+\gamma) n \geq K_{0}$.

Considere uma 3-coloração arbitrária $c: E\left(K_{N}\right) \rightarrow[3]$ das arestas de $K_{N}$ e subgrafos geradores $G_{1}, G_{2}$ e $G_{3}$ de $K_{N}$, onde $i j \in E\left(G_{s}\right)$ se e somente se $c(i j)=s$, para $s=1,2,3$. Pelo Lema de Regularidade, sabemos que existe uma partição $\left(V_{0}, V_{1}, \ldots, V_{k}\right)$ dos vértices de $K_{N}$ tal que

$$
\left|V_{i}\right|=m \geq(1-\varepsilon) \frac{N}{k}
$$

para $1 \leq i \leq k$, e pelo menos $(1-\varepsilon)\left(\begin{array}{l}k \\ 2\end{array}\right)$ pares $\left\{V_{i}, V_{j}\right\}$, para $1 \leq i<j \leq k$, são $\varepsilon$-regulares em todos os três grafos $G_{1}, G_{2}$ e $G_{3}$, onde $k_{0} \leq k \leq K_{0}$.

Definimos o seguinte grafo reduzido: seja $R$ o grafo com conjunto de vértices $[k]$, onde $i j \in E(R)$ se e somente se $\left\{V_{i}, V_{j}\right\}$ é $\varepsilon$-regular em $G_{1}, G_{2}$ e $G_{3}$. Assim, $|E(R)| \geq(1-\varepsilon)\left(\begin{array}{l}k \\ 2\end{array}\right)$. Portanto, sabemos que $R$ é um grafo com $k$ vértices e densidade pelo menos $(1-\varepsilon)$. Agora, defina a coloração $c^{\prime}: E(R) \rightarrow[3]$ das arestas de $R$ de modo que $c^{\prime}(i, j)=s$ se $s \in[3]$ é o maior inteiro tal que $\left|E_{G_{s}}\left(V_{i}, V_{j}\right)\right| \geq\left|E_{G_{r}}\left(V_{i}, V_{j}\right)\right|$ para $1 \leq r \leq 3$, i.e., a aresta $i j$ recebe uma 
das cores que mais aparece nas arestas de $E_{K_{N}}\left(V_{i}, V_{j}\right)$ com relação à coloração $c$ de $E\left(K_{N}\right)$. Claramente, se $c^{\prime}(i j)=s$, então $\left|E_{G_{s}}\left(V_{i}, V_{j}\right)\right| \geq\left|V_{i}\right|\left|V_{j}\right| / 3$.

Como $k \geq k_{0}$ e a densidade de $R$ é pelo menos $(1-\varepsilon)$, pelo Lema 4.10 , sabemos que $R$ contém uma árvore monocromática $T$ que contém um emparelhamento $E_{M}$ de tamanho $\ell \geq(1-\delta) k / 4$. Sem perda de generalidade, supomos que as arestas de $T$ são coloridas com a cor 1. Pela Afirmativa 4.11, podemos rotular $E_{M}=\left\{x_{i} y_{i}: i=1, \ldots, \ell\right\}$ de modo que $x_{i}$ e $x_{j}$ estão a uma distância par em $T$, para $1 \leq i<j \leq \ell$.

Sejam $\left\{z_{1}, \ldots, z_{\ell^{\prime}}\right\}$ os vértices de $T$ que não são cobertos por arestas do emparelhamento $E_{M}$. Como todas as arestas de $T$ estão presentes em $R$, sabemos que, para todas as arestas $i j \in E(T)$, os pares $\left\{V_{i}, V_{j}\right\}$ são $\varepsilon$-regulares em $G_{1}$ com $\left|E_{G_{1}}\left(V_{i}, V_{j}\right)\right| \geq\left|V_{i}\right|\left|V_{j}\right| / 3$. Assim, o lema está provado, uma vez que podemos considerar o grafo $G$ composto pelas classes $V_{i}$, para todo $i \in V(T)$, pondo $E(G)\left(V_{i}, V_{j}\right)=E_{G_{1}}\left(V_{i}, V_{j}\right)$ para todos os pares $\left(V_{i}, V_{j}\right)$, onde $i, j \in V(T)$.

\subsubsection{Intervalos balanceados}

Seja $H$ um grafo que admite uma 2-coloração de seus vértices que utiliza as 2 cores aproximadamente a mesma quantidade de vezes, no total. Esse fato pode não ser válido localmente. Nesta seção mostramos como balancear um grafo $H$ de modo que as duas cores sejam utilizadas, localmente, aproximadamente a mesma quantidade de vezes.

Dado um grafo $H=(W, E)$, com $W=\left\{w_{1}, \ldots, w_{n}\right\}$ e uma 2-coloração $\chi: W \rightarrow[2]$, defina a função $C_{i}^{\chi}$ (ou $C_{i}$ ) tal que, se $W^{\prime} \subset W$, então $C_{i}\left(W^{\prime}\right)=\left|\chi^{-1}(i) \cap W^{\prime}\right|$ para $i=1,2$. Isto é, $C_{i}\left(W^{\prime}\right)$ é a quantidade de vértices de $W^{\prime}$ que recebem a cor $i$ da coloração $\chi$. Dizemos que $\chi$ é uma coloração $\beta$-balanceada de $W$ se temos $\left|C_{1}^{\chi}(W)-C_{2}^{\chi}(W)\right| \leq \beta C_{2}^{\chi}(W)$. Um subconjunto $I \subset W$ é chamado de intervalo se existem naturais $p$ e $q$, com $p \leq q$ tais que $I=\left\{w_{p}, w_{p+1}, \ldots, w_{q}\right\}$. Finalmente, sejam $\ell^{\prime}$ e $\hat{\ell}$ inteiros positivos com $\ell^{\prime} \leq \hat{\ell}$ e seja $\sigma:\left[\ell^{\prime}\right] \rightarrow[\hat{\ell}]$ uma função injetora. Considere uma partição de $W$ em um conjunto de intervalos $\mathcal{I}=\left\{I_{1}, \ldots, I_{\hat{\ell}}\right\}$. Definimos $C_{i}(\mathcal{I}, \sigma, a)=\sum_{j=1}^{a} C_{i}\left(I_{\sigma(j)}\right)$ para $i=1,2$. Se é claro qual é a partição de intervalos estamos considerando, então escrevemos simplesmente $C_{i}(\sigma, a)$.

O lema abaixo diz que, dada uma 2-coloração suficientemente balanceada dos vértices de um grafo $H$, toda partição de $V_{H}$ em intervalos de aproximadamente o mesmo tamanho pode ser reordenada de modo que, após a ordenação, se excluirmos qualquer quantidade de intervalos no final da ordenação, então, nos vértices restantes, a quantidade de vértices que recebem a cor 1 não difere muito da quantidade de vértices que recebem a cor 2 .

Lema 4.13. Para todo inteiro $\hat{\ell} \geq 1$, existe $n_{0}$ tal que, se $H=(W, E)$ é um grafo com $W=\left\{w_{1}, \ldots, w_{n}\right\}$, onde $n \geq n_{0}$, então para toda 2 -coloração $\beta$-balanceada $\chi$ de $W$ com $\beta \leq 2 / \hat{\ell}$, e toda partição de $W$ em intervalos $I_{1}, \ldots, I_{\hat{\ell}}$ com $\left|I_{1}\right| \leq \ldots \leq\left|I_{\hat{\ell}}\right| \leq\left|I_{1}\right|+1$, existe uma permutação $\sigma:[\hat{\ell}] \rightarrow[\hat{\ell}]$ tal que, para $i=1, \ldots, \hat{\ell}$, temos

$$
\left|C_{1}(\sigma, i)-C_{2}(\sigma, i)\right| \leq \frac{n}{\hat{\ell}}+1
$$


Demonstração. Fixe $\hat{\ell} \geq 1$ e tome $n_{0}=2 \hat{\ell}^{3}$. Seja $H=(W, E)$ um grafo com conjunto de vértices $W=\left\{w_{1}, \ldots, w_{n}\right\}$, onde $n \geq n_{0}$. Fixe uma 2-coloração $\beta$-balanceada $\chi$ de $W$, onde $\beta \leq 2 / \hat{\ell}$, e uma partição de $W$ em intervalos $I_{1}, \ldots, I_{\hat{\ell}} \operatorname{com}\left|I_{1}\right| \leq \ldots \leq\left|I_{\hat{\ell}}\right| \leq\left|I_{1}\right|+1$.

Vamos construir uma permutação $\sigma$ iterativamente. Podemos pegar qualquer inteiro em $[\hat{\ell}]$ para ser $\sigma(1)$, pois o tamanho dos intervalos é no máximo $n / \hat{\ell}+1$. Agora, suponha que tenhamos definido $\sigma(1), \ldots, \sigma(i)$ de modo que $\left|C_{1}(\sigma, i)-C_{2}(\sigma, i)\right| \leq n / \hat{\ell}+1$, onde $i \leq \hat{\ell}-1$.

Se $C_{1}(\sigma, i)=C_{2}(\sigma, i)$, então claramente $\sigma(i+1)$ pode ser definido como sendo qualquer dos inteiros remanescentes em $[\hat{\ell}]$. Assim, sem perda de generalidade, assuma que $C_{1}(\sigma, i)=C_{2}(\sigma, i)+k$, onde $1 \leq k \leq n / \hat{\ell}+1$. Mas, como $C_{1}(\sigma, i)+C_{2}(\sigma, i) \leq i(n / \hat{\ell}+1)$, podemos concluir que

$$
C_{2}(\sigma, i) \leq \frac{i n}{2 \hat{\ell}}-\frac{k-i}{2}
$$

Iremos mostrar que existe algum $r \in[\hat{\ell}] \backslash \bigcup_{j=1}^{i} \sigma(j)$ tal que $C_{2}\left(I_{r}\right) \geq k / 2$. Suponha, por contradição, que $C_{2}\left(I_{r}\right)<k / 2$ para todos os $\hat{\ell}-i$ inteiros $r \in[\hat{\ell}] \backslash \bigcup_{j=1}^{i} \sigma(j)$. Esse fato, juntamente com (4.3), implica o seguinte.

$$
\begin{aligned}
C_{2}(W) & <C_{2}(\sigma, i)+(\hat{\ell}-i) \frac{k}{2} \\
& =\frac{i n}{2 \hat{\ell}}+(\hat{\ell}-i-1) \frac{k}{2}+\frac{i}{2} \\
& \leq\left(\frac{\hat{\ell}-1}{\hat{\ell}}\right) \frac{n}{2}+\frac{\hat{\ell}}{2} \\
& =\left(1-\left(\frac{1}{\hat{\ell}}-\frac{\hat{\ell}}{n}\right)\right) \frac{n}{2},
\end{aligned}
$$

onde a última desigualdade segue de $k \leq n / \hat{\ell}+1$ e $i \leq \hat{\ell}-1$. Como $C_{1}(W)+C_{2}(W)=n$, utilizando (4.4), concluímos que

$$
\begin{aligned}
\frac{C_{1}(W)}{C_{2}(W)} & =\frac{n}{C_{2}(W)}-1 \\
& >1+\frac{2\left(n-\hat{\ell}^{2}\right)}{n(\hat{\ell}-1)+\hat{\ell}^{2}} \\
& \geq 1+\beta,
\end{aligned}
$$

onde a última desigualdade segue da escolha de $n_{0}$, dado que $\beta \leq 2 / \hat{\ell}$. Mas isso contradiz o $\beta$-balanceamento da 2-coloração $\chi$ de $W$. Portanto, sabemos que existe $r \in[\hat{\ell}] \backslash \bigcup_{j=1}^{i} \sigma(j)$ 
com $C_{2}\left(I_{r}\right) \geq k / 2$. Tome $\sigma(i+1)=r$. Então

$$
\begin{aligned}
C_{1}(\sigma, i+1) & =C_{1}(\sigma, i)+C_{1}\left(I_{r}\right) \\
& \leq\left(C_{2}(\sigma, i)+k\right)+\left(\frac{n}{\hat{\ell}}+1-\frac{k}{2}\right) \\
& =\left(C_{2}(\sigma, i)+\frac{k}{2}\right)+\frac{n}{\hat{\ell}}+1 \\
& \leq C_{2}(\sigma, i+1)+\frac{n}{\hat{\ell}}+1 .
\end{aligned}
$$

Pela desigualdade acima, e notando que, claramente, $C_{1}(\sigma, i+1) \geq C_{2}(\sigma, i+1)-(n / \hat{\ell}+1)$, concluímos que $\left|C_{1}(\sigma, i+1)-C_{2}(\sigma, i+1)\right| \leq n / \hat{\ell}+1$.

Seja $H=(W, E)$ um grafo com $W=\left\{w_{1}, \ldots, w_{n}\right\}$ e seja $\chi: W \rightarrow[2]$ uma 2-coloração de $W$. Considere uma partição de $W$ definida pelos intervalos $\mathcal{I}=\left\{I_{1}, \ldots, I_{\hat{\ell}}\right\}$. Definimos $C_{i}(\mathcal{I}, \sigma, a, b)=\sum_{j=a}^{b} C_{i}\left(I_{\sigma(j)}\right)$ para $i=1,2$. Se é claro qual a partição de intervalos que estamos considerando, então escrevemos somente $C_{i}(\sigma, a, b)$.

O seguinte corolário diz que, após a reordenação dos intervalos de vértices de $H$, a quantidade de vértices com a cor 1 não difere muito da quantidade de vértices com a cor 2 em qualquer intervalo formado por intervalos de vértices reordenados.

Corolário 4.14. Para todo inteiro $\hat{\ell} \geq 1$, existe $n_{0}$ tal que, se $H=(W, E)$ é um grafo com $W=\left\{w_{1}, \ldots, w_{n}\right\}$, onde $n \geq n_{0}$, então para toda 2 -coloração $\beta$-balanceada $\chi$ de $W$ com $\beta \leq 2 / \hat{\ell}$, e para toda partição de $W$ em intervalos $I_{1}, \ldots, I_{\hat{\ell}}$ com $\left|I_{1}\right| \leq \ldots \leq\left|I_{\hat{\ell}}\right| \leq\left|I_{1}\right|+1$, existe uma permutação $\sigma:[\hat{\ell}] \rightarrow[\hat{\ell}]$ tal que, para todos os inteiros $1 \leq a<b \leq \hat{\ell}$, temos

$$
\left|C_{1}(\sigma, a, b)-C_{2}(\sigma, a, b)\right| \leq 2\left(\frac{n}{\hat{\ell}}+1\right)
$$

Demonstração. Fixe $\hat{\ell} \geq 1$ e seja $n_{0}$ obtido pelo Lema 4.13 aplicado com $\hat{\ell}$. Seja $H=(W, E)$ o grafo com conjunto de vértices $W=\left\{w_{1}, \ldots, w_{n}\right\}$, onde $n \geq n_{0}$. Fixe uma 2-coloração $\beta$-balanceada $\chi$ de $W$ e uma partição de $W$ em intervalos $I_{1}, \ldots, I_{\hat{\ell}}$ tal que os tamanhos dos intervalos satisfazem $\left|I_{1}\right| \leq \ldots \leq\left|I_{\hat{\ell}}\right| \leq\left|I_{1}\right|+1$, onde $\beta \leq 2 / \hat{\ell}$.

Seja $\sigma$ a permutação dada pelo Lema 4.13 . Fixe inteiros $1 \leq a<b \leq \hat{\ell}$ e suponha s.p.g. que $C_{1}(\sigma, a, b) \geq C_{2}(\sigma, a, b)$. Portanto, pela conclusão do Lema 4.13 , temos

$$
\begin{aligned}
C_{1}(\sigma, a, b) & =C_{1}(\sigma, b)-C_{1}(\sigma, a-1) \\
& \leq\left(C_{2}(\sigma, b)+n / \hat{\ell}+1\right)-\left(C_{2}(\sigma, a-1)-(n / \hat{\ell}+1)\right) \\
& =C_{2}(\sigma, a, b)+2(n / \hat{\ell}+1) .
\end{aligned}
$$

O lema seguinte, resultado principal desta seção, afirma que, para toda 2-coloração balanceada $\chi$ de $V_{H}$ e para qualquer partição de $V_{H}$ em intervalos, é possível reordenar esses 
intervalos, de modo que $\chi$ é balanceada em qualquer intervalo suficientemente grande formado pela união dos intervalos de vértices de $H$. Esse resultado garante o balanceamento local que precisamos para aplicar o Lema de Explosão.

Lema 4.15. Para todo $\xi>0$ e todo inteiro $\hat{\ell} \geq 1$, existe $n_{0}$ tal que se $H=(W, E)$ é um grafo com $W=\left\{w_{1}, \ldots, w_{n}\right\}$, onde $n \geq n_{0}$, então para toda 2 -coloração $\beta$-balanceada $\chi$ de $W$ com $\beta \leq 2 / \hat{\ell}$, e para toda partição de $W$ em intervalos $I_{1}, \ldots, I_{\hat{\ell}}$ com $\left|I_{1}\right| \leq \ldots \leq\left|I_{\hat{\ell}}\right| \leq\left|I_{1}\right|+1$ existe uma permutação $\sigma:[\hat{\ell}] \rightarrow[\hat{\ell}]$ tal que, para todos os inteiros $1 \leq a<b \leq \hat{\ell}$ com $b-a \geq 7 / \xi$, temos

$$
\left|C_{1}(\sigma, a, b)-C_{2}(\sigma, a, b)\right| \leq \xi C_{2}(\sigma, a, b),
$$

Demonstração. Fixe $\xi>0, \hat{\ell} \geq 1$ e seja $n_{0}$ obtido pelo Corolário 4.14 aplicado com $\hat{\ell}$. Seja $H=(W, E)$ um grafo com $W=\left\{w_{1}, \ldots, w_{n}\right\}$, onde $n \geq \max \left\{n_{0},(4 / 3) \hat{\ell}\right\}$, e fixe uma 2-coloração $\beta$-balanceada $\chi$ de $W$, onde $\beta \leq 2 / \hat{\ell}$, e uma partição de $W$ em intervalos $I_{1}, \ldots, I_{\hat{\ell}}, \operatorname{com}\left|I_{1}\right| \leq \ldots \leq\left|I_{\hat{\ell}}\right| \leq\left|I_{1}\right|+1$.

Seja $\sigma$ a permutação dada pelo Corolário 4.14. Fixe inteiros $1 \leq a<b \leq \hat{\ell}$ tais que $b-a>7 / \xi$. Lembre que a conclusão do Corolário 4.14 implica que

$$
\left|C_{1}(\sigma, a, b)-C_{2}(\sigma, a, b)\right| \leq 2(n / \hat{\ell}+1)
$$

A desigualdade acima e o fato de $C_{1}(\sigma, a, b)+C_{2}(\sigma, a, b)=(b-a) n / \hat{\ell}$ implicam que

$$
C_{2}(\sigma, a, b) \geq\left(\frac{b-a}{2}\right) \frac{n}{\hat{\ell}}-(n / \hat{\ell}+1)
$$

Pela escolha de $a, b$ e $n_{0}$, temos

$$
C_{2}(\sigma, a, b) \geq(2 / \xi)(n / \hat{\ell}+1)
$$

Combinando as desigualdades (4.6) e (4.7), concluímos a prova.

\subsection{Prova do Teorema 3-Ramsey}

Antes de detalhar a prova, explicamos rapidamente de que modo conectaremos os resultados discutidos na Seção 4.2. Para uma abordagem mais geral sobre as ideias envolvidas na prova do Teorema 3-Ramsey (Teorema 4.3), veja a Seção 4.1.

Para todo $\gamma>0$ e todo $n$ suficientemente grande, dada uma 3-coloração arbitrária das arestas de $K_{N}$, para $N=(2+\gamma) n$, provaremos que se $H$ é um $(\beta, \Delta)$-bigrafo balanceado com $n$ vértices, então $K_{N}$ possui uma cópia monocromática de $H$.

A estratégia para provar o Teorema 4.3 é aplicar o Lema de Explosão (Lema 4.7) para encontrar a cópia desejada de $H$ em $K_{N}$. Para fazer isso, utilizaremos o Lema 4.12 para encontrar um subgrafo monocromático $G$ de $K_{N}$ composto por pares regulares suficientemente 
densos. Assim, utilizando as Afirmativas 4.8 e 4.9, é fácil ver que, removendo poucos vértices de $G$, podemos encontrar um subgrafo monocromático $G^{\prime} \subset G$ que contém uma partição regular contendo pares super-regulares que cobrem $(1+o(1)) n$ vértices.

Por fim, construímos uma partição de $V_{H}$ e fazemos uso do Lema 4.15 para mostrar que essa partição é compatível com a partição regular de $G^{\prime}$. Assim, podemos aplicar o Lema de Explosão para encontrar a cópia monocromática de $H$, concluindo a prova.

\section{Prova do Teorema 3-Ramsey (Teorema 4.3)}

Fixe $\gamma>0$ e um natural $\Delta \geq 1$. O Lema 4.12, aplicado com $\gamma$, fornece $\varepsilon_{0}$. Agora, aplicamos o Lema 4.7 com parâmetros $d=1 / 4$ e $\Delta$, recebendo $\varepsilon_{1}$. Defina

$$
\varepsilon=\min \left\{\varepsilon_{0}, \varepsilon_{1} / 2, \gamma / 19\right\}
$$

Como $\varepsilon \leq \varepsilon_{0}$, Lema 4.12 fornece um natural $K_{0}$. Fixe $\xi=\gamma / 304$ e seja $n_{0}$ dado por uma aplicação do Lema 4.15 com parâmetros $\xi$ e $K_{0}$. Defina

$$
\beta=\varepsilon \xi(1+2 \xi) / 36 \Delta^{2} K_{0}^{2}
$$

Seja $H=\left(W, E_{H}\right)$ um $(\beta, \Delta)$-bigrafo balanceado com $n \geq \max \left\{n_{0}, K_{0}\right\} /(2+\gamma)$ vértices. Defina $N=\lfloor(2+\gamma) n\rfloor$ e considere uma coloração arbitrária $\chi_{K_{N}}: E\left(K_{N}\right) \rightarrow[3]$. Nosso objetivo é mostrar que a coloração $\chi_{K_{N}}$ leva a uma cópia monocromática de $H$.

Particionando os vértices de $K_{N}$.

Vamos encontrar um subgrafo monocromático $G^{\prime}$ de $K_{N}$ suficientemente regular. Pelo Lema 4.12 sabemos que existe uma cor (digamos que seja a cor 1 ), inteiros $\ell, \ell^{\prime}, k$ com $\ell, \ell^{\prime} \leq k \leq K_{0}$ e $\ell \geq(1-\gamma / 4) k / 4$, uma árvore $T$ com conjunto de vértices

$$
V(T)=\left\{x_{1}, \ldots, x_{\ell}, y_{1}, \ldots, y_{\ell}, z_{1}, \ldots, z_{\ell^{\prime}}\right\}
$$

contendo um emparelhamento $E_{M}=\left\{x_{i} y_{i}: i=1, \ldots, \ell\right\}$, onde a distância entre quaisquer $x_{i}$ e $x_{j}$ em $T$ é par, tal que existe uma partição $\left(V_{i}\right)_{i \in[k]}$ de $V=V_{K_{N}}$, onde $K_{N}^{1}$ é $(\varepsilon, 1 / 3)$ regular em $T$ e $\left|V_{1}\right|=\ldots=\left|V_{k}\right|=m \geq(1-\varepsilon) N / k$. Seja $G_{T}$ o subgrafo de $K_{N}^{1}$ induzido pelas classes em $\left(V_{i}\right)_{i \in[k]}$ que correspondem aos vértices de $T$.

Para aplicar o Lema de Explosão, precisamos que as classes de $G_{T}$ que correspondem às arestas do emparelhamento $E_{M}$ formem pares super-regulares, e os outros pares de classes sejam suficientemente regulares. Podemos garantir isso deletando alguns poucos vértices de $G_{T}$. De fato, aplicando a Afirmativa 4.9 e depois aplicando a Afirmativa 4.8 . é fácil ver que encontramos um subgrafo $G_{T}^{\prime} \subset G_{T}$ com classes $A_{1}, \ldots, A_{\ell}, B_{1}, \ldots, B_{\ell}$, $C_{1}, \ldots, C_{\ell^{\prime}}$ de tamanho pelo menos $(1-\varepsilon) m$, correspondendo, respectivamente, aos vér- 
tices $x_{1}, \ldots, x_{\ell}, y_{1}, \ldots, y_{\ell}, z_{1}, \ldots, z_{\ell^{\prime}}$ da árvore $T$, de modo que os grafos bipartidos induzidos por $A_{i}$ e $B_{i}$ são $(2 \varepsilon, 1 / 3-\varepsilon)$-super-regulares, e os grafos bipartidos induzidos por todos os outros pares são $(2 \varepsilon, 1 / 3-\varepsilon)$-regulares. Ademais, seja $D_{\min }$ o conjunto de menor cardinalidade dentre os conjuntos $A_{1}, \ldots, A_{\ell}, B_{1}, \ldots, B_{\ell}, C_{1}, \ldots, C_{\ell^{\prime}}$. Como $\varepsilon \leq \gamma / 19, m \geq(1-\varepsilon) N / k$ e $\ell \geq(1-\gamma / 4) k / 4$, podemos facilmente verificar que

$$
\left|D_{\min }\right| \geq(1+\gamma / 152) n / 2 \ell
$$

Particionando os vértices de $H$.

Agora iremos construir uma partição de $W$ pronta para uma aplicação do Lema 4.7 . Como $H$ é um $(\beta, \Delta)$-bigrafo balanceado, sabemos que existe uma coloração $\chi: V_{H} \rightarrow[2]$ tal que ||$\chi^{-1}(1)|-| \chi^{-1}(2)|| \leq \beta\left|\chi^{-1}(2)\right|$.

Seja $\left(w_{1}, \ldots, w_{n}\right)$ uma ordenação de $W$ que respeita a largura de banda, i.e., $|i-j| \leq \beta n$ para toda aresta $w_{i} w_{j} \in E_{H}$. Defina $\hat{\ell}$ como o menor inteiro dividindo $n$ tal que $\hat{\ell} \geq$ $\left(7 K_{0} / \xi\right)+\ell \geq \ell(7 / \xi+1)$. Considere a partição de $V_{H}$ em intervalos $I_{1}, \ldots, I_{\hat{\ell}}$, com $\left|I_{1}\right|=$ $\ldots=\left|I_{\hat{\ell}}\right|=n / \hat{\ell}$, levando em conta a ordenação $\left(w_{1}, \ldots, w_{n}\right)$, i.e., $I_{i}=w_{(i-1) n / \hat{\ell}+1}, \ldots, w_{i n / \hat{\ell}}$ para $i=1, \ldots, \hat{\ell}$. Pelo Lema 4.15 , como $\beta \leq 2 / \hat{\ell}$, existe uma permutação $\sigma:[\hat{\ell}] \rightarrow[\hat{\ell}]$ tal que

$$
\left|C_{1}(\sigma, a, b)-C_{2}(\sigma, a, b)\right| \leq \xi C_{2}(\sigma, a, b)
$$

para todos os inteiros $1 \leq a<b \leq \hat{\ell} \operatorname{com} b-a \geq 7 / \xi$. Defina $a_{i}=(i-1) \hat{\ell} / \ell+1$ e $b_{i}=i \hat{\ell} / \ell$ e considere os blocos $J_{i}=\left\{I_{\sigma\left(a_{i}\right)}, I_{\sigma\left(a_{i}+1\right)}, \ldots, I_{\sigma\left(b_{i}\right)}\right\}$ para $i=1, \ldots, \ell$. Por simplicidade, escrevemos $C_{1}\left(J_{i}\right)$ para $C_{1}\left(\sigma, a_{i}, b_{i}\right)$ e $C_{2}\left(J_{i}\right)$ para $C_{2}\left(\sigma, a_{i}, b_{i}\right)$. Assim, para $i=1, \ldots, \ell$, como $b_{i}-a_{i}=\hat{\ell} / \ell-1 \geq 7 / \xi$, temos

$$
\left|C_{1}\left(J_{i}\right)-C_{2}\left(J_{i}\right)\right| \leq \xi C_{2}\left(J_{i}\right)
$$

Lembre que a árvore $T$ possui conjunto de vértices $\left\{x_{1}, \ldots, x_{\ell}, y_{1}, \ldots, y_{\ell}, z_{1}, \ldots, z_{\ell^{\prime}}\right\}$ e contém o emparelhamento $M=\left\{x_{i} y_{i}: i=1, \ldots, \ell\right\}$, onde a distância em $T$ entre quaisquer $x_{i}$ e $x_{j}$ é par. A partição de $W$ será composta por classes $X_{1}, \ldots, X_{\ell}, Y_{1}, \ldots, Y_{\ell}, Z_{1}, \ldots, Z_{\ell^{\prime}}$ que correspondem, respectivamente, aos vértices $x_{1}, \ldots, x_{\ell}, y_{1}, \ldots, y_{\ell}, z_{1}, \ldots, z_{\ell^{\prime}}$.

para todo $i \in[\ell]$, colocaremos a maioria dos vértices de $J_{i}$ nas classes $X_{i}$ e $Y_{i}$, dependendo da cor que recebem de $\chi$. Os restantes serão distribuídos de modo a tornar possível 'caminhar' entre as classes $X_{i}$ e $Y_{i}$. Vamos nos referir às classes $X_{i}$ e $Y_{i}$ como classes emparelhadas.

Dividimos cada intervalo $I_{i}$ em duas partes. A primeira, chamada de conector de $I_{i}$, é denotada por $\mathrm{CON}_{i}$. Os conectores são responsáveis por fazer a conexão entre duas classes emparelhadas. Pomos $\mathrm{CON}_{\hat{\ell}}=\emptyset$. Para $1 \leq i \leq \hat{\ell}-1$, se $I_{i}$ e $I_{i+1}$ estão no mesmo bloco $J_{r}$, então $\mathrm{CON}_{i}=\emptyset$. Suponha agora que $I_{i} \in J_{r}$ e $I_{i+1} \in J_{s} \operatorname{com} r \neq s$ e $1 \leq i \leq \hat{\ell}-1$. Seja $P_{T}(r, s)$ o caminho em $T$ entre $x_{r}$ e $x_{s}$, e considere o caminho $P_{T}^{\prime}(r, s) \subset P_{T}(r, s)$, 
obtido a partir da remoção dos vértices do conjunto $\left\{x_{r}, y_{r}, x_{s}, y_{s}\right\}$ de $P_{T}(r, s)$, i.e., $P_{T}^{\prime}(r, s)$ é a parte 'interna' do caminho de $T$ que é utilizado para alcançar $x_{s}$ a partir de $x_{r}$. Por simplicidade, faça $t_{r, s}=\left|P_{T}^{\prime}(r, s)\right|$. Nesse caso, dividimos os $\left(t_{r, s}+1\right) \beta n$ últimos vértices de $I_{i}$ em $t_{r, s}+1$ 'pedaços' de tamanho $\beta n$, obedecendo a sequência em que estão no intervalo. O $j$-ésimo pedaço é denotado por $\mathrm{CON}_{i}(j)$ para $1 \leq j \leq t_{r, s}+1$. Pomos $\mathrm{CON}_{i}=\left\{\mathrm{CON}_{i}(1), \ldots, \mathrm{CON}_{i}\left(t_{r, s}\right), \mathrm{CON}_{i}\left(t_{r, s}+1\right)\right\}$

Agora que já descrevemos os conectores, podemos definir a parte principal dos intervalos. Definimos $\mathrm{NUC}_{i}=I_{i} \backslash \mathrm{CON}_{i}$ como o núcleo do intervalo $I_{i}$, que será colocado nas classes emparelhadas $X_{i}$ e $Y_{i}$.

Construiremos agora as classes que irão compor a partição de $V_{H}$. Inicialmente, cada classe em $\left\{X_{1}, \ldots, X_{\ell}, Y_{1}, \ldots, Y_{\ell}, Z_{1}, \ldots, Z_{\ell^{\prime}}\right\}$ está vazia. Para cada $1 \leq i \leq \ell$, considere o bloco $J_{i}$. Agora, para cada intervalo $I_{p} \in J_{i}$, incluímos em $X_{i}$ todos os vértices $w$ do núcleo $\mathrm{NUC}_{p}$ com $\chi(w)=1$ e incluímos em $Y_{i}$ todos os vértices $w$ de $\mathrm{NUC}_{p}$ com $\chi(w)=2$.

O próximo passo é acomodar todos os vértices que estão em conectores. Fixe $1 \leq i \leq \hat{\ell}-1$. Iremos acomodar os conectores de $I_{i}$. Assuma que $I_{i}$ está contido em $J_{r}$ e que $I_{i+1}$ está contido em $J_{s}$ com $r \neq s$, caso contrário, os conectores que estamos considerando são vazios e não há nada a fazer. Seja $\left\{u_{1}, \ldots, u_{t_{r, s}}\right\}$ o caminho interno $P_{T}^{\prime}(r, s)$ e seja $u_{0}$ e $u_{t_{r, s}+1}$, respectivamente, os vértices de $T$ conectados a $u_{1}$ e $u_{t_{r, s}}$ em $P_{T}(r, s)$. Lembre que a árvore $T$ possui conjunto de vértices $\left\{x_{1}, \ldots, x_{\ell}, y_{1}, \ldots, y_{\ell}, z_{1}, \ldots, z_{\ell^{\prime}}\right\}$. Sem perda de generalidade, suponha que $u_{0}=x_{r}$. Então, colocamos os vértices $w$ de $\operatorname{CON}_{i}(1)$ com $\chi(w)=1 \mathrm{em} X_{r}$ e aqueles com $\chi(w)=2$ nós colocamos na classe correspondente a $u_{1}$. Agora, pomos os vértices $w$ de $\operatorname{CON}_{i}(2)$ com $\chi(v)=2$ na classe correspondente a $u_{1}$ e aqueles com $\chi(w)=1$ nós colocamos na classe correspondente a $u_{2}$. Continuando o procedimento, ponha os vértices $w$ de $\mathrm{CON}_{i}(3) \operatorname{com} \chi(w)=1$ na classe correspondente a $u_{2}$ e aqueles com $\chi(w)=2$ nós colocamos na classe correspondente a $u_{3}$, e assim por diante, até que precisamos cuidar dos vértices em $\mathrm{CON}_{i}\left(t_{r, s}\right)$. Suponha, s.p.g., que $t_{r, s}$ é par. Assim, como $x_{r}$ e $x_{s}$ estão à distância par em $T$, sabemos que $w_{t_{r, s}+1}=y_{s}$. Assim, para o último pedaço do conector de $I_{i}$, colocamos os vértices $w$ de $\operatorname{CON}_{i}\left(t_{r, s}+1\right)$ com $\chi(w)=1$ na classe correspondente a $u_{t_{r, s}}$ e aqueles com $\chi(w)=2$ colocamos em $Y_{s}$. Note que não existem arestas dentro das classes, e se existem arestas entre duas classes, então a aresta correspondente está presente em $T$.

\section{Aplicando o Lema de Explosão.}

Seja $M$ o grafo com o mesmo conjunto de vértices de $T$ e com conjunto de arestas $E_{M}$ (Lembre que $E_{M}$ é o emparelhamento obtido por nossa aplicação do Lema 4.12). Iremos mostrar que a partição de $V_{H}$ que construímos é $\left(2 \varepsilon_{1}, T, M\right)$-compatível com a partição de $V_{G^{\prime}}$ que construímos. Assim, podemos aplicar o Lema de Explosão para encontrar a cópia desejada de $H$ em $K_{N}$.

Primeiramente, limitamos por cima o tamanho de cada classe na partição de $V_{H}$ dada pelas classes $X_{1}, \ldots, X_{\ell}, Y_{1}, \ldots, Y_{\ell}, Z_{1}, \ldots, Z_{\ell^{\prime}}$. Para todo $1 \leq i \leq \ell$ sabemos que $C_{1}\left(J_{i}\right)+$ 
$C_{2}\left(J_{i}\right)=n / \ell$. Utilizando esse fato e 4.9 , podemos facilmente obter que, para todo $1 \leq i \leq \ell$,

$$
(1-\xi) \frac{n}{2 \ell} \leq C_{1}\left(J_{i}\right), C_{2}\left(J_{i}\right) \leq(1+\xi) \frac{n}{2 \ell}
$$

Por construção, todo $X_{i}\left(Y_{i}\right)$ é composto somente por vértices $v$ com $\chi(v)=1(\chi(v)=2)$. Ademais, esses vértices podem vir dos núcleos dos intervalos de $J_{i}$ e de no máximo dois pedaços de cada conector. Assim,

$$
\begin{aligned}
\left|X_{i}\right|,\left|Y_{i}\right| & \leq(1+\xi) \frac{n}{2 \ell}+2 \hat{\ell} \beta n \\
& =(1+\xi+4 \hat{\ell} \beta) \frac{n}{2 \ell} \\
& \leq\left|D_{\min }\right|,
\end{aligned}
$$

onde a última desigualdade segue de 4.8 e da escolha de $\xi$ e $\beta$.

Para as classes $Z_{i}$ com $1 \leq i \leq \ell^{\prime}$, sabemos que essas classes são compostas somente por vértices em no máximo dois pedaços de cada conector. Logo,

$$
\begin{aligned}
\left|Z_{i}\right| & \leq 2 \hat{\ell} \beta n \\
& =(4 \hat{\ell} \ell \beta) \frac{n}{2 \ell} \\
& \leq \frac{\varepsilon}{\Delta^{2}}\left|D_{\min }\right|
\end{aligned}
$$

onde a última desigualdade segue de (4.8) e da escolha de $\beta$.

Agora podemos verificar que as partições de $V_{H}$ e $V_{G^{\prime}}$ que construímos são compatíveis. Baseado na Definição 4.6, definimos conjuntos $U_{j}^{\prime}$, para $1 \leq j \leq 2 \ell+\ell^{\prime}$, relacionados à partição $\left\{X_{1}, \ldots, X_{\ell}, Y_{1}, \ldots, Y_{\ell}, Z_{1}, \ldots, Z_{\ell^{\prime}}\right\}$ de $V_{H}$. Ponha $W_{j}=X_{j}$ se $1 \leq j \leq \ell$, defina $W_{j}=Y_{j-\ell}$ se $\ell+1 \leq j \leq 2 \ell$, e defina $W_{j}=Z_{j-2 \ell}$ se $2 \ell+1 \leq j \leq 2 \ell+\ell^{\prime}$. Iremos verificar que as quatro condições da Definição 4.6 são satisfeitas:

(i): Pela construção da partição de $V_{H}$, se existe uma aresta entre duas classes, então a aresta correspondente está presente em $T$.

(ii): Por 4.8, sabemos que cada conjunto $D$ em $\left\{A_{1}, \ldots, A_{\ell}, B_{1}, \ldots, B_{\ell}, C_{1}, \ldots, C_{\ell^{\prime}}\right\}$ possui tamanho $|D| \geq\left|D_{\min }\right|$. Assim, as desigualdades 4.11) e 4.12 confirmam que a condição (ii) é satisfeita.

(iii): Fixe $1 \leq j \leq 2 \ell+\ell^{\prime}$. Defina $U_{j}$ como o conjunto de vértices em $W_{j}$ com vizinhos em algum $W_{k}$ com $j \neq k$ e $\{j, k\} \notin M$. Considere os seguintes casos.

(a) $2 \ell+1 \leq j \leq 2 \ell+\ell^{\prime}$ : Temos $U_{j}=Z_{j-2 \ell}$. Por $4.12,\left|U_{j}\right| \leq \varepsilon\left|D_{\min }\right| / \Delta$.

(b) $1 \leq j \leq 2 \ell$ : Neste caso, $U_{j}$ é composto somente por vizinhos de vértices em exatamente um conjunto em $\left\{Z_{1}, \ldots, Z_{\ell^{\prime}}\right\}$. Assim, como $\Delta$ é o grau máximo de $H$, por 4.12, concluímos que $\left|U_{j}\right| \leq \varepsilon\left|D_{\min }\right| / \Delta$. 
Portanto, para todo $j=1, \ldots, 2 \ell+\ell^{\prime}$, temos

$$
\left|U_{j}\right| \leq \frac{\varepsilon}{\Delta}\left|D_{\min }\right|
$$

o que confirma que a condição (iii) é satisfeita.

(iv): Defina o conjunto $U_{j}^{\prime}=N_{H}(U) \cap\left(W_{j} \backslash U\right)$, onde $U=\bigcup_{i=1}^{2 \ell+\ell^{\prime}} U_{i}$. Considere os seguintes casos.

(a) $2 \ell+1 \leq j \leq 2 \ell+\ell^{\prime}$ : Note que $U_{j}^{\prime}=\emptyset$ se $2 \ell+1 \leq j \leq 2 \ell+\ell^{\prime}$, pois cada vértice de $Z_{j-2 \ell}$ pertence a $U_{j}$. Assim, é óbvio que $\left|U_{j}^{\prime}\right| \leq \varepsilon\left|D_{\min }\right|$.

(b) $\ell+1 \leq j \leq 2 \ell$ : Aqui, temos $U_{j}^{\prime} \subset W_{j}=Y_{j-\ell}$. Logo, $U_{j}^{\prime}$ é composto somente por vizinhos de vértices em $U_{j-\ell} \subset X_{j-\ell}$. Portanto, utilizando 4.13, obtemos $\left|U_{j}^{\prime}\right| \leq \Delta\left|U_{j-\ell}\right| \leq \varepsilon\left|D_{\min }\right|$.

(c) $1 \leq i \leq \ell$ : Este caso é análogo ao anterior.

Isso mostra que a condição (iv) é satisfeita.

Portanto, provamos que as quatro condições da Definição 4.6 são satisfeitas. Assim, a partição $\left\{X_{1}, \ldots, X_{\ell}, Y_{1}, \ldots, Y_{\ell}, Z_{1}, \ldots, Z_{\ell^{\prime}}\right\}$ de $V_{H}$ é $(2 \varepsilon, T, M)$-compatível (logo, é $\left(\varepsilon_{1}, T, M\right)$ compatível) com a partição $\left\{A_{1}, \ldots, A_{\ell}, B_{1}, \ldots, B_{\ell}, C_{1}, \ldots, C_{\ell^{\prime}}\right\}$ de $V_{G^{\prime}}$. Com isso, usamos o Lema 4.7 para concluir que $H \subset G^{\prime}$, finalizando a prova, dado que $G^{\prime}$ é um subgrafo monocromático de $K_{N}$.

\subsection{Ideia da prova do Teorema 2-Ramsey}

Queremos provar que, para todo $\gamma>0$ e inteiro positivo $\Delta$, existe uma constante $\beta>0$ tal que, para todo $(\beta, \Delta)$-bigrafo $H$ suficientemente grande com uma 2-coloração própria $\chi: V_{H} \rightarrow[2]$, onde $t_{1}=\left|\chi^{-1}(1)\right|$ e $t_{2}=\left|\chi^{-1}(2)\right|$, com $t_{1} \leq t_{2}$, podemos encontrar uma cópia monocromática de $H$ em toda 2-coloração das arestas de $K_{N}$, onde aqui consideramos $N=(1+\gamma) \max \left\{2 t_{1}+t_{2}, 2 t_{2}\right\}$. Seja $H$ um grafo satisfazendo essas condições e suponha que $2 t_{1} \geq t_{2}$.

A prova do Teorema 4.2 é semelhante à prova do Teorema 4.3. de modo que, para o Teorema 4.2, também realizamos a imersão de $H$ em partes, considerando uma partição de um subgrafo monocromático $G_{T}$ de $K_{N}$. A partição que precisamos construir deve ser composta por uma classe especial $W$ e classes $X_{1}, Y_{1}, \ldots, X_{m}, Y_{m}$ correspondendo a um emparelhamento $M=\left\{x_{i}, y_{i}: i=1, \ldots, m\right\}$ 'grande', de modo que todos os pares $\left\{X_{i}, Y_{i}\right\}$ são super-regulares e os pares $\left\{X_{i}, W\right\}$ são regulares, para $i=1, \ldots, m$.

O problema na preparação do grafo monocromático $G_{T}$ é o fato de $H$ não ser balanceado, como no contexto do Teorema 4.3. Assim, para fazer a imersão de $H$ em $G_{T}$, precisamos que $\left|Y_{i}\right| /\left|X_{i}\right|=t_{2} / t_{1}$. Felizmente, por um resultado de [32], como $t_{2} / t_{1} \leq 1 / 2$ no caso que estamos 
considerando, podemos achar tal partição de $V_{G}$. Pela Afirmativa 4.9, podemos facilmente tornar os pares correspondentes às arestas do emparelhamento em pares super-regulares.

Agora precisamos preparar o grafo $H$ para a imersão. Consideramos a ordenação dos vértices de $H$ respeitando a condição de largura de banda e dividimos seu conjunto de vértices em intervalos. Assim, podemos encontrar uma permutação de tais intervalos de modo que blocos de intervalos 'cabem' nos pares super-regulares de $G_{T}$. Por fim, é possível mostrar que, utilizando poucos vértices, é possível 'caminhar' de um par super-regular a outro, como feito no Teorema 4.3 . Com isso, a prova é finalizada. 


\section{Capítulo 5}

\section{Considerações finais}

O primeiro problema estudado nesta tese é relacionado à imersão em hipergrafos pseudoaleatórios. Objetivamos determinar a quantidade de cópias de certos hipergrafos fixos em hipergrafos suficientemente pseudoaleatórios. Outro tipo de problema abordado neste trabalho faz parte da clássica Teoria de Ramsey, onde deseja-se determinar o menor tamanho que um grafo completo pode ter, garantindo a existência de cópias monocromáticas de certos grafos em uma coloração arbitrária de suas arestas.

Com relação aos problemas de imersão em hipergrafos, provamos um lema de contagem que generaliza um resultado de Kohayakawa, Rödl e Sissokho [37]. Mostramos que, dado um hipergrafo $k$-uniforme $H$, linear e livre de conectores, a quantidade de cópias de $H$ em um hipergrafo suficientemente pseudoaleatório $G$ é $(1+o(1)) n^{|V(H)|} p^{|E(H)|}$, desde que $p \gg n^{-1 / D_{H}}$ e $|E(G)|=\left(\begin{array}{l}n \\ k\end{array}\right) p$.

Seria interessante investigar algumas aplicações do lema de imersão provado neste trabalho. Acreditamos que seja possível introduzir um lema de regularidade para hipergrafos que, juntamente com o lema de imersão apresentado, nos permita obter resultados relacionados à imersão de hipergrafos fixos em hipergrafos maiores. Dizemos que um grafo $G=(V, E)$ satisfaz a propriedade $\mathcal{Q}(\eta, \delta, \alpha)$ se todo subgrafo $G[S]$ induzido por $S \subset V$ com $|S| \geq \eta|V|$ é tal que $(\alpha-\delta)\left(\begin{array}{c}|S| \\ 2\end{array}\right)<|E(G[S])|<(\alpha+\delta)\left(\begin{array}{c}|S| \\ 2\end{array}\right)$. Talvez seja possível estender, para hipergrafos, o seguinte resultado provado em [50]:

Teorema 5.1. Para todos $k \geq 1$ e $\alpha, \eta>0$, existem $\delta>0$ e um inteiro $n_{0}>0$ tais que para todo $n \geq n_{0}$ vale o seguinte: se $G$ é um grafo com $n$ vértices que satisfaz a propriedade $\mathcal{Q}(\eta, \delta, \alpha)$, então $G$ contém todos os grafos com $k$ vértices como subgrafos induzidos.

Provamos também dois resultados na área de Teoria de Ramsey. Determinamos assintoticamente o número de Ramsey de ordem dois para para grafos bipartidos com largura de banda pequena e grau máximo limitado, e o número de Ramsey de ordem três para os mesmos grafos bipartidos, mas com a hipótese adicional de que ambas as classes do grafo bipartido possuam aproximadamente o mesmo tamanho. Como corolário desses dois resultados, mostramos que, para grades $G_{a, b}$, temos $R\left(G_{a, b}, G_{a, b}\right)=(3 / 2+o(1)) a b$ e $R\left(G_{a, b}, G_{a, b}, G_{a, b}\right)=(2+o(1)) a b$, onde $o(1)$ tende a zero quando $a b$ tende a infinito. 
Acreditamos que, através do uso das técnicas utilizadas para determinar os números de Ramsey para grafos bipartidos com largura de banda pequena e grau máximo limitado, seja possível obter resultados similares para outras classes de grafos. Por exemplo, partindo de uma recente extensão do conhecido "Blow-up Lemma" [4], esperamos obter alguns resultados para grafos $a$-ordenáveis, i.e., grafos tais que seus vértices podem ser ordenados como $\left(v_{1}, \ldots, v_{n}\right)$ de modo que $\left|N\left(N\left(v_{i}, D_{i}\right), E_{i}\right)\right| \leq a$ para todo $1 \leq i \leq n$, onde $E_{i}=\left\{v_{1}, \ldots, v_{i}\right\}$ e $D_{i}=\left\{v_{i+1}, \ldots, v_{n}\right\}$. Não é difícil ver que todo grafo $G \operatorname{com} \Delta(G) \leq a$ é $\left(a^{2}-a+1\right)$ ordenável. Por fim, sabe-se também que grafos planares são 10-ordenáveis. 


\section{Referências Bibliográficas}

[1] P. Allen, G. Brightwell, and J. Skokan, Ramsey-goodness - and otherwise, Combinatorica, to appear.

[2] J. Balogh, R. Morris, and W. Samotij, Independent sets in hypergraphs, 2011, submitted.

[3] F. S. Benevides and J. Skokan, The 3-colored Ramsey number of even cycles, J. Combin. Theory Ser. B 99 (2009), no. 4, 690-708.

[4] J. Böttcher, Y. Kohayakawa, A. Taraz, and A. Würfl, An extension of the blow-up lemma to arrangeable graphs, ArXiv e-prints (2013).

[5] J. Böttcher, Embedding large graphs - the Bollobás-Komlós conjecture and beyond, Ph.D. thesis, Technische Universität München, 2009.

[6] J. Böttcher, P. Heinig, and A. Taraz, Embedding into bipartite graphs, SIAM J. Discrete Math. 24 (2010), no. 4, 1215-1233.

[7] J. Böttcher, K. P. Pruessmann, A. Taraz, and A. Würfl, Bandwidth, expansion, treewidth, separators and universality for bounded-degree graphs, European J. Combin. 31 (2010), no. 5, 1217-1227.

[8] F. Chung and R. Graham, Sparse quasi-random graphs, Combinatorica 22 (2002), no. 2, 217-244, Special issue: Paul Erdős and his mathematics.

[9] _ Quasi-random graphs with given degree sequences, Random Structures Algorithms 32 (2008), no. 1, 1-19.

[10] F. Chung, R. Graham, and R. Wilson, Quasirandom graphs, Proc. Nat. Acad. Sci. U.S.A. 85 (1988), no. 4, 969-970.

[11] D. Conlon, The ramsey number of dense graphs, ArXiv e-prints (2009).

[12] D. Conlon, J. Fox, C. Lee, and B. Sudakov, Ramsey numbers of cubes versus cliques, ArXiv e-prints (2012).

[13] D. Conlon, J. Fox, and Y. Zhao, Extremal results in sparse pseudorandom graphs, ArXiv e-prints (2012).

[14] _ A relative Szemerédi theorem, ArXiv e-prints (2013).

[15] D. Conlon, T. Gowers, W. Samotij, and M. Schacht, On the KŁR conjecture in random graphs, submitted.

[16] D. Conlon, J. Fox, and B. Sudakov, On two problems in graph Ramsey theory, Combinatorica 32 (2012), no. 5, 513-535. 
[17] R. J. Faudree and R. H. Schelp, Path Ramsey numbers in multicolorings, J. Combinatorial Theory Ser. B 19 (1975), no. 2, 150-160.

[18] A. Figaj and T. Łuczak, The Ramsey number for a triple of long even cycles, J. Combin. Theory Ser. B 97 (2007), no. 4, 584-596.

[19] G. Fiz Pontiveros, S. Griffiths, and R. Morris, The triangle-free process and $R(3, k)$, ArXiv e-prints (2013).

[20] G. Fiz Pontiveros, S. Griffiths, R. Morris, D. Saxton, and J. Skokan, The Ramsey number of the clique and the hypercube, ArXiv e-prints (2013).

[21] A. Frieze and M. Krivelevich, Packing Hamilton cycles in random and pseudo-random hypergraphs, Random Structures Algorithms 41 (2012), no. 1, 1-22.

[22] A. Frieze, M. Krivelevich, and P.-S. Loh, Packing tight Hamilton cycles in 3-uniform hypergraphs, Random Structures Algorithms 40 (2012), no. 3, 269-300.

[23] Z. Füredi, Random Ramsey graphs for the four-cycle, Discrete Math. 126 (1994), no. 13, 407-410.

[24] L. Gerencsér and A. Gyárfás, On Ramsey-type problems, Ann. Univ. Sci. Budapest. Eötvös Sect. Math. 10 (1967), 167-170.

[25] S. Gerke, H. J. Prömel, T. Schickinger, A. Steger, and A. Taraz, $K_{4}$-free subgraphs of random graphs revisited, Combinatorica 27 (2007), no. 3, 329-365.

[26] S. Gerke, T. Schickinger, and A. Steger, $K_{5}$-free subgraphs of random graphs, Random Structures Algorithms 24 (2004), no. 2, 194-232.

[27] S. Gerke and A. Steger, The sparse regularity lemma and its applications, Surveys in combinatorics 2005, London Math. Soc. Lecture Note Ser., vol. 327, Cambridge Univ. Press, Cambridge, 2005, pp. 227-258.

[28] R. L. Graham, B. L. Rothschild, and J. H. Spencer, Ramsey theory, second ed., WileyInterscience Series in Discrete Mathematics and Optimization, John Wiley \& Sons Inc., New York, 1990, A Wiley-Interscience Publication.

[29] A. Gyárfás, M. Ruszinkó, G. N. Sárközy, and E. Szemerédi, Three-color Ramsey numbers for paths, Combinatorica 27 (2007), no. 1, 35-69.

[30] _ Tripartite Ramsey numbers for paths, J. Graph Theory 55 (2007), no. 2, 164174.

[31] P. E. Haxell, T. Łuczak, Y. Peng, V. Rödl, A. Ruciński, M. Simonovits, and J. Skokan, The Ramsey number of hypergraph cycles. I, J. Combin. Theory Ser. A 113 (2006), no. 1, 67-83.

[32] P. E. Haxell, T. Euczak, and P. W. Tingley, Ramsey numbers for trees of small maximum degree, Combinatorica 22 (2002), no. 2, 287-320, Special issue: Paul Erdős and his mathematics.

[33] Y. Kohayakawa, Szemerédi's regularity lemma for sparse graphs, Foundations of computational mathematics (Rio de Janeiro, 1997), Springer, Berlin, 1997, pp. 216-230. 
[34] Y. Kohayakawa and B. Kreuter, Threshold functions for asymmetric Ramsey properties involving cycles, Random Structures Algorithms 11 (1997), no. 3, 245-276.

[35] Y. Kohayakawa, T. Łuczak, and V. Rödl, On $K^{4}$-free subgraphs of random graphs, Combinatorica 17 (1997), no. 2, 173-213.

[36] Y. Kohayakawa and V. Rödl, Szemerédi's regularity lemma and quasi-randomness, Recent advances in algorithms and combinatorics, CMS Books Math./Ouvrages Math. SMC, vol. 11, Springer, New York, 2003, pp. 289-351.

[37] Y. Kohayakawa, V. Rödl, and P. Sissokho, Embedding graphs with bounded degree in sparse pseudorandom graphs, Israel J. Math. 139 (2004), 93-137.

[38] Y. Kohayakawa, T. Łuczak, and V. Rödl, Arithmetic progressions of length three in subsets of a random set, Acta Arith. 75 (1996), no. 2, 133-163.

[39] Y. Kohayakawa, V. Rödl, M. Schacht, and J. Skokan, On the triangle removal lemma for subgraphs of sparse pseudorandom graphs, An irregular mind, Bolyai Soc. Math. Stud., vol. 21, János Bolyai Math. Soc., Budapest, 2010, pp. 359-404.

[40] J. Komlós and M. Simonovits, Szemerédi's regularity lemma and its applications in graph theory, Combinatorics, Paul Erdős is eighty, Vol. 2 (Keszthely, 1993), Bolyai Soc. Math. Stud., vol. 2, János Bolyai Math. Soc., Budapest, 1996, pp. 295-352.

[41] J. Komlós, G. N. Sárközy, and E. Szemerédi, Blow-up lemma, Combinatorica 17 (1997), no. $1,109-123$.

[42] J. Komlós, G. N. Sárközy, and E. Szemerédi, An algorithmic version of the blow-up lemma, Random Structures Algorithms 12 (1998), no. 3, 297-312.

[43] J. Komlós, A. Shokoufandeh, M. Simonovits, and E. Szemerédi, The regularity lemma and its applications in graph theory, Theoretical aspects of computer science (Tehran, 2000), Lecture Notes in Comput. Sci., vol. 2292, Springer, Berlin, 2002, pp. 84-112.

[44] M. Krivelevich and B. Sudakov, Pseudo-random graphs, More sets, graphs and numbers, Bolyai Soc. Math. Stud., vol. 15, Springer, Berlin, 2006, pp. 199-262.

[45] D. Kühn, D. Osthus, and A. Taraz, Large planar subgraphs in dense graphs, J. Combin. Theory Ser. B 95 (2005), no. 2, 263-282.

[46] T. Euczak, $R\left(C_{n}, C_{n}, C_{n}\right) \leq(4+o(1)) n$, J. Combin. Theory Ser. B 75 (1999), no. 2, 174-187.

[47] S. P. Radziszowski, Small Ramsey numbers, Electron. J. Combin. 1 (1994 (Lastest update: 2011)), Dynamic Survey 1, 84 pp. (electronic).

[48] F. P. Ramsey, On a problem of formal logic, Proc. London Math. Soc. 30 (1930), 264286.

[49] V. Rödl, A. Ruciński, and A. Taraz, Hypergraph packing and graph embedding, Combin. Probab. Comput. 8 (1999), no. 4, 363-376, Random graphs and combinatorial structures (Oberwolfach, 1997).

[50] V. Rödl, On universality of graphs with uniformly distributed edges, Discrete Math. 59 (1986), no. 1-2, 125-134. 
[51] V. Rödl and A. Ruciński, Perfect matchings in E-regular graphs and the blow-up lemma, Combinatorica 19 (1999), no. 3, 437-452.

[52] D. Saxton and A. Thomason, Hypergraph containers, 2013, submitted.

[53] E. Szemerédi, Regular partitions of graphs, Problèmes combinatoires et théorie des graphes (Colloq. Internat. CNRS, Univ. Orsay, Orsay, 1976), Colloq. Internat. CNRS, vol. 260, CNRS, Paris, 1978, pp. 399-401.

[54] A. Thomason, Pseudorandom graphs, Random graphs '85 (Poznań, 1985), NorthHolland Math. Stud., vol. 144, North-Holland, Amsterdam, 1987, pp. 307-331. 